Florida International University FIU Digital Commons

9-12-2013

\title{
Distribution of Light in the Human Retina under Natural Viewing Conditions
}

Jorge C. Gibert

Florida International University, jgiber01@fiu.edu

DOI: $10.25148 /$ etd.FI13112204

Follow this and additional works at: https://digitalcommons.fiu.edu/etd

Part of the Physics Commons

\section{Recommended Citation}

Gibert, Jorge C., "Distribution of Light in the Human Retina under Natural Viewing Conditions" (2013). FIU Electronic Theses and Dissertations. 958.

https://digitalcommons.fiu.edu/etd/958

This work is brought to you for free and open access by the University Graduate School at FIU Digital Commons. It has been accepted for inclusion in FIU Electronic Theses and Dissertations by an authorized administrator of FIU Digital Commons. For more information, please contact dcc@fiu.edu. 


\section{FLORIDA INTERNATIONAL UNIVERSITY}

Miami, Florida

\section{DISTRIBUTION OF LIGHT IN THE \\ HUMAN RETINA UNDER NATURAL VIEWING CONDITIONS}

A dissertation submitted in partial fulfillment of the

requirements for the degree of

DOCTOR OF PHILOSOPHY

in

PHYSICS

by

Jorge C. Gibert 
To: Dean Kenneth G. Furton

College of Arts and Sciences

This dissertation, written by Jorge C. Gibert, and entitled Distribution of Light in the Human Retina under Natural Viewing Conditions, having been approved in respect to style and intellectual content, is referred to you for judgment.

We have read this dissertation and recommend that it be approved.

Bernard S. Gerstman

John T. Landrum

Rajamani Narayanan

Richard A. Bone, Major Professor

Date of Defense: September 12, 2013

The dissertation of Jorge C. Gibert is approved.

Dean Kenneth G. Furton College of Arts and Sciences

Dean Lakshmi N. Reddi University Graduate School

Florida International University, 2013 


\section{DEDICATION}

I dedicate this dissertation to my wife Luz, my children George and Grace, and my mother Farah. This dissertation is also dedicated to Richard Bone for he is the best man I've ever known. 


\section{ACKNOWLEDGEMENTS}

I would like to express my profound appreciation and gratitude to my mentor Dr. Richard Bone, for his incommensurable patience, and the guidance and support he provided me throughout this study. Dr. Bone's non-verbal way of teaching humility has helped me to become not only a better scientist but also a better person.

I would like to thank my committee members Dr. Bernard S. Gerstman, Dr. John T. Landrum and Dr. Rajamani Narayanan for their continuous help and support. Particularly, Dr. Gerstman questioning made my defense particularly exciting. I thank Dr. Landrum for his direct and honest method of encouragement he has been a true guide in all my years in the biophysics group. Dr. Narayanan's help and support was crucial in dealing with the dissertation process.

Especial thanks to Dr. Lair Kramer for supporting part of my research thought the PhysTec grant. His help has been very important in my development as a physics instructor.

I would like to thank Carlos Orta, Wuenlue "Norman" Huang, Manuel Wong, for their help in the experimental set-up.

This research was supported in part by the Florida International University MBRS RISE program, NIH grant SC3GM083671, and Four Leaf Japan Ltd 
ABSTRACT OF THE DISSERTATION

DISTRIBUTION OF LIGHT IN THE

HUMAN RETINA UNDER NATURAL VIEWING CONDITIONS

by

Jorge C. Gibert

Florida International University, 2013

Miami, Florida

Professor Richard A. Bone, Major Professor

Age-related macular degeneration (AMD) is the leading cause of blindness in America. The fact that AMD wreaks most of the damage in the center of the retina raises the question of whether light, integrated over long periods, is more concentrated in the macula.

A method, based on eye-tracking, was developed to measure the distribution of light in the retina under natural viewing conditions. The hypothesis was that integrated over time, retinal illumination peaked in the macula. Additionally a possible relationship between age and retinal illumination was investigated.

The eye tracker superimposed the subject's gaze position on a video recorded by a scene camera. Five informed subjects were employed in feasibility tests, and 58 naïve subjects participated in 5 phases. In phase 1 the subjects viewed a gray-scale image. In phase 2, they observed a sequence of photographic images. In phase 3 they viewed a video. In phase 4, they worked on a computer; in phase 5, the subjects walked around freely. The informed subjects were instructed to gaze at bright objects in the field of view and then at dark objects. Naïve subjects were allowed to gaze freely for all phases. Using the subject's gaze coordinates, and the video provided by the scene camera, the cumulative light distribution on the retina was calculated for $\sim 15^{\circ}$ around the fovea.

As expected for control subjects, cumulative retinal light distributions peaked and dipped in the fovea when they gazed at bright or dark objects respectively. The light distribution maps obtained 
from the naïve subjects presented a tendency to peak in the macula for phases 1,2 , and 3 , a consistent tendency in phase 4 and a variable tendency in phase 5 .

The feasibility of using an eye-tracker system to measure the distribution of light in the retina was demonstrated, thus helping to understand the role played by light exposure in the etiology of AMD. Results showed that a tendency for light to peak in the macula is a characteristic of some individuals and of certain tasks. In these situations, risk of AMD could be increased. No significant difference was observed based on age. 


\section{TABLE OF CONTENTS}

\section{CHAPTER}

PAGE

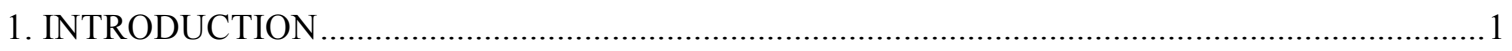

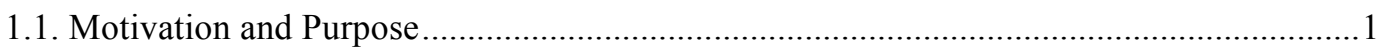

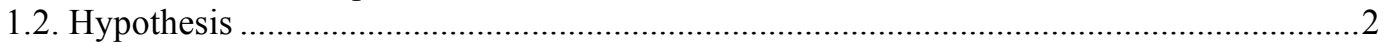

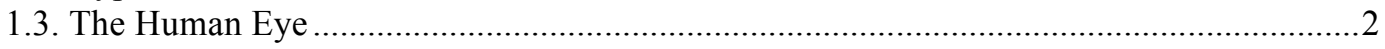

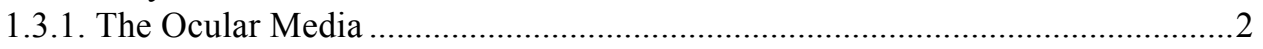

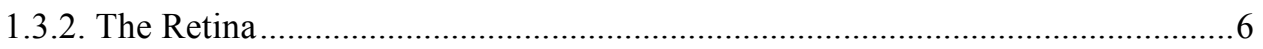

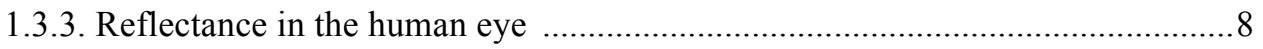

1.3.4. Spectral Reflection and Absorption in the Human Eye ......................................

1.4. Age-Related Macular Degeneration ......................................................................... 14

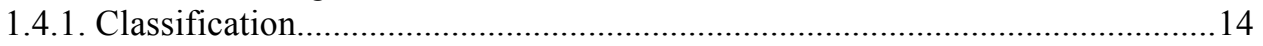

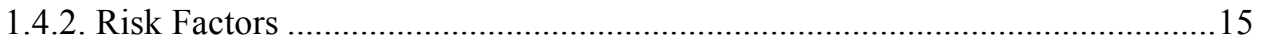

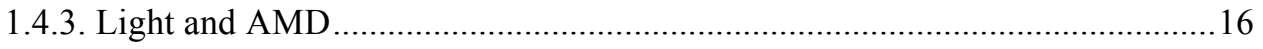

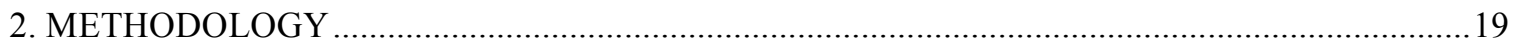

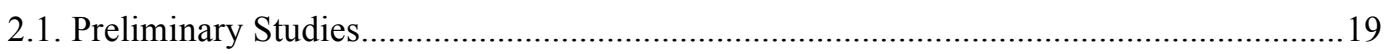

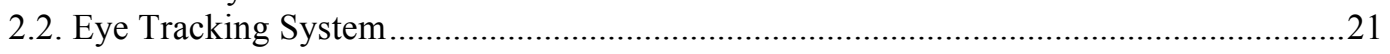

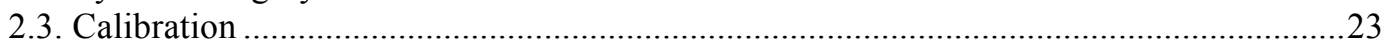

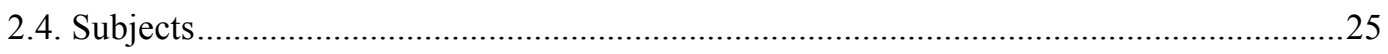

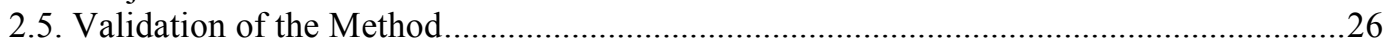

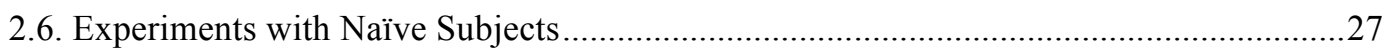

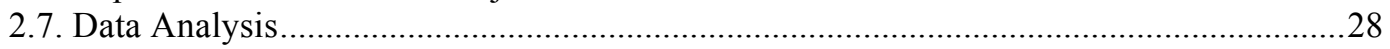

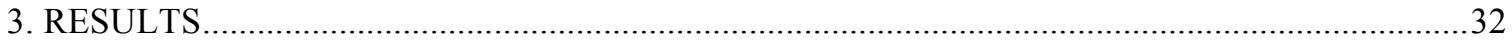

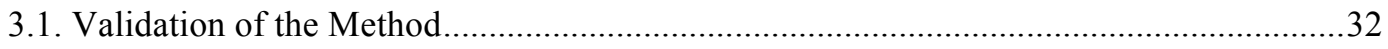

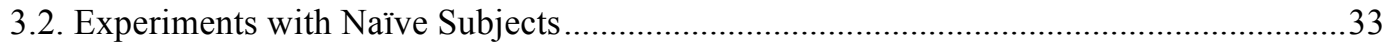

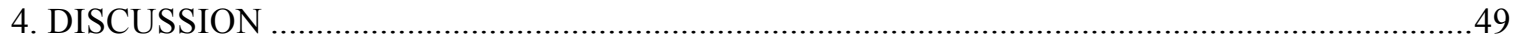

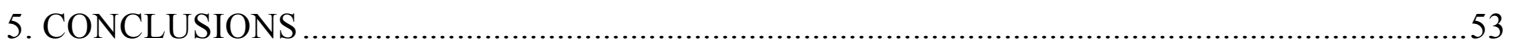

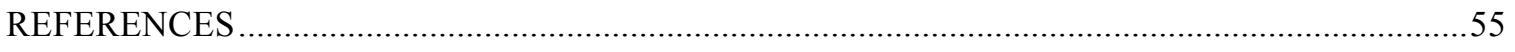

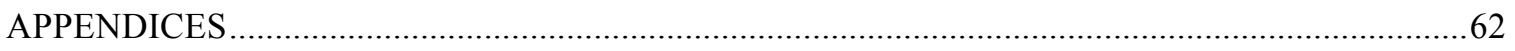

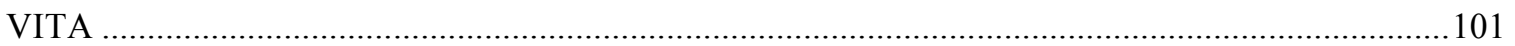




\section{LIST OF TABLES}

TABLE

PAGE

3.1. Analysis of the mean values of the difference in retinal illuminance within $1^{\circ}$ around the fovea

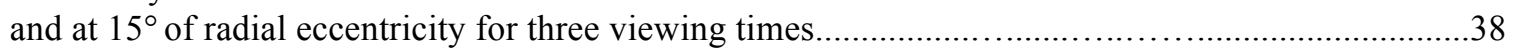

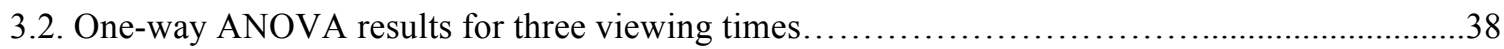

3.3. Multiple comparison post hoc Turkey's test for three viewing times....................................39

3.4. Analysis of the mean values of the difference in retinal illuminance within $1^{\circ}$ around the fovea and at $15^{\circ}$ of radial eccentricity for three age groups................................................................

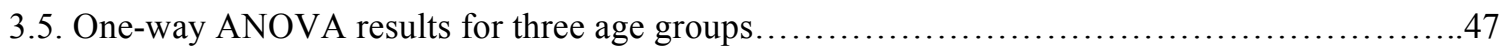

3.6. Multiple comparison post hoc Turkey's test for three age groups $\ldots \ldots \ldots \ldots \ldots \ldots \ldots \ldots \ldots \ldots \ldots \ldots$ 


\section{LIST OF FIGURES}

FIGURE

PAGE

1.1. Schematic median section of the eye seen from above. 1-Cornea; 2-aqueous humor; 3-crystalline lens; 4-vitreous humor; 5-retina; 6-choroids; 7-sclera; 8-optic nerve; 9-fovea; 10-optic disk; 11-ora serrata; 12-ciliary muscle; 13-zonule fibers; 14- iris; 15-ocular conjunctiva. (Wyszecki \& Stiles, 1982, p. 82)...

1.2. Photographic image of the retina in blue light. The macula is the highly pigmented region at the center.

1.3. Approximate distribution of rods and cones across the retina. The solid and dotted lines represent the cones and rods respectively (adapted from Osterberg, 1935, p.89).

1.4. Structure of the human fovea (Polyak, 1941, p.69)............................................

1.5. Schema of the model of reflectances and absorptions in the human eye. (Van Norren-Tiemeijer, 1986, p. 316).

1.6. Absorption spectra of blood, melanin, macular pigment, lens, and water in the eye for a single pass

1.7. Representative chromatograms of photopigments extracted from three different regions of retinal tissue. (A) $7.1 \mathrm{~mm} 2$ disk centered on the fovea. (B) $93-\mathrm{mm} 2$ annulus. (C) $343 \mathrm{~mm} 2$ annulus. $\mathrm{L}=$ Lutein, $\mathrm{Z}=$ Zeaxanthin (Bone et al. 1988, p. 539).

1.8. Relative optical density distributions at $460 \mathrm{~nm}$ of (a) macular pigment, (b) cone photopigment, (c) rod photopigment, and d) melanin along a horizontal meridian through the fovea. (Bone et al., 2007, p. 3275)

1.9. Relative Ganzfeld illumination of the retina as a function of field angle without considering media extinction. Results from Kooijman's all-aspheric, Pflibsen, and Kooijman's all-spherical (Adapted from Pflibsen et al, 1988).

2.1. Modified eye-tracker (SensoMotoric Instruments SMI ${ }^{\circledR}$ ) used for preliminary studies....

2.2. Simple test used in the preliminary study (a) visual stimulus. (b) pictorial representation of the retinal illuminance when the subject fixated on the white region in (a) horizontal line-scan through (b) showing a peak illuminance at the center of the retina. .20

2.3. Test used in preliminary studies. (a) Image freely viewed by subject for 1 minute (b) Resulting retinal light distribution (c) Vertical line scan through center of (b) ..............................20

2.4. Eye-Tracking system provided by Arrington Research ${ }^{\circledR}$

2.5. Video frame recorded by the eye-camera showing the dark pupil and the corneal glint. The system measures the vector length between the center of the pupil (shown in red) and the center of the corneal glint. . . 
2.6 Step-and-increment calibration mode. (a)Video frame recorded by the scene camera in "GazeSpace" window. The point being calibrated appears in blue. (b) "EyeSpace" window image showing a successful calibration. The point being calibrated appears white .......................24

2.7. "Flat-white" image used for vignetting correction. (a) actual image (b) intensity map............26

2.8. Example of vignetting correction (a) uncorrected image (b) corrected image ..........................26

2.9. Hypothetical example showing a video frame projected on the retina. Dimensions are

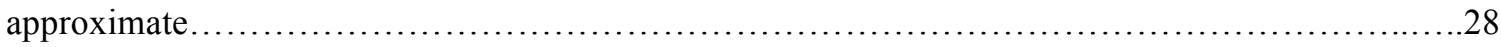

2.10. Data recording and processing. (a) Four actual frames as recorded by the scene camera. Gaze position is indicated by the green circle. (b) Intensity maps produced by the region bounded by the

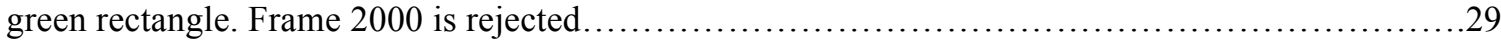

2.11. Intensity maps from Fig. 2.11b. The green line indicates the subject's gaze positions............30

3.1. Mesh plot showing the cumulative light distribution (relative) on the retina for an informed subject instructed to look at the brightest objects in the field of view while walking freely around the lab. 32

3.2. Mesh plot showing the cumulative light distribution (relative) on the retina for an informed subject instructed to look at the darkest objects in the field of view while walking freely around the lab. .32

3.3. Intensity maps showing the average cumulative light distribution (relative) on the retina for naïve subjects viewing the gray-scale shown in Fig.3.3a. (a) 16 subjects (b) 11 subjects (c) 3 subjects. 34

3.4. Map showing the average cumulative light distribution (relative) on the retina for naïve subjects viewing a gray-scale image .34

3.5. (a) Mean values versus radial eccentricity $\left( \pm 0.25^{\circ}\right)$ derived from Fig. 3. (b) Horizontal line-scan

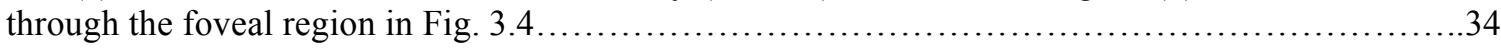

3.6. Intensity maps showing the average cumulative light distribution (relative) on the retina for naïve subjects viewing a PowerPoint presentation of photographic images. (a) 11 subjects (b) 8 subjects....35

3.7. Map showing the average cumulative light distribution (relative) on the retina for naïve subjects viewing a PowerPoint presentation of photographic images. .35

3.8. (a) Mean values versus radial eccentricity $\left( \pm 0.25^{\circ}\right)$ derived from Fig. 3. (b) Horizontal line-scan

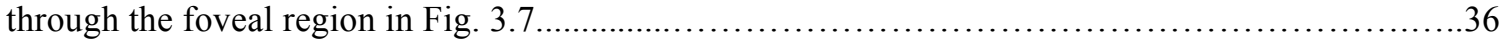

3.9. Intensity maps showing the average cumulative light distribution (relative) on the retina for naïve subjects viewing a video presentation. (a) 4 subjects (b) 8 subjects (c) 3 subjects .35

3.10. Map showing the average cumulative light distribution (relative) on the retina for naïve subjects viewing a video presentation. 36

3.11. (a) Mean values versus radial eccentricity $\left( \pm 0.25^{\circ}\right)$ derived from Fig. 3.10. (b) Horizontal linescan through the foveal region in Fig. 3.10. 
3.12. Map showing the average cumulative light distribution (relative) on the retina for naïve subjects viewing a video computer monitor.

3.13. (a) Mean values versus radial eccentricity $\left( \pm 0.5^{\circ}\right)$ derived from Fig. 3.12. (b) Horizontal linescan through the foveal region in Fig. 3.12 showing clear maximum at the foveal center................38

3.14. Intensity maps showing the average cumulative light distribution (relative) on the retina for naïve subjects walking freely around the lab and surrounding hallways (a) 10 subjects (b) 8 subjects

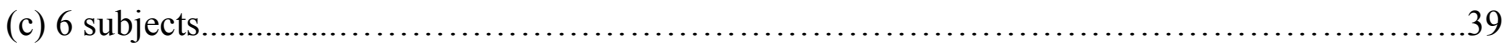

3.15. Map showing the average cumulative light distribution (relative) on the retina for naïve subjects walking freely around the lab and surrounding hallways................................................................

3.16. (a) Mean values versus radial eccentricity $\left( \pm 0.25^{\circ}\right)$ derived from Fig. 3.15. (b) Horizontal line-

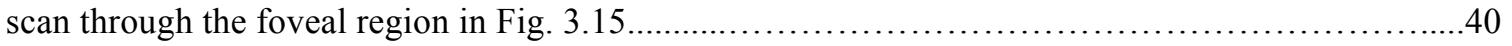

3.17. Intensity maps showing the average cumulative light distribution (relative) on the retina for naïve subjects walking freely around campus (a) 2 subjects (b) 3 subjects (c) 5 subjects....................41

3.18. Map showing the average cumulative light distribution (relative) on the retina for naïve subjects walking freely around campus. . .41

3.19. (a) Mean values versus radial eccentricity $\left( \pm 0.25^{\circ}\right)$ derived from Fig 4.18 (b) horizontal line-

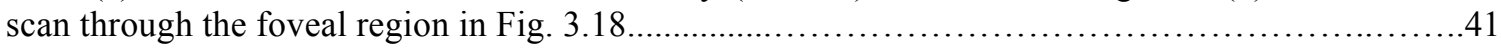

3.20. Average retinal illuminance maps of all subjects combined...................................42

3.21. (a) Mean retinal illumination versus eccentricity from Fig. 3.20. (b) Horizontal line-scan through the foveal region in Fig. 3.20.

3.22. (a) Mean retinal illumination versus eccentricity from Fig. 3.20. (b) Horizontal line-scan through

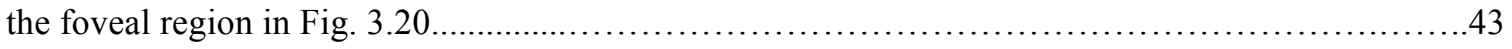

3.23. Average map of the results obtained from two of the experiments in which the head of the

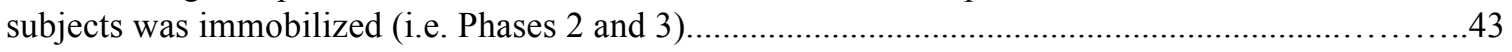

3.24. (a) Mean retinal illumination versus eccentricity derived from Fig. 3.22. (b) Horizontal line-scan

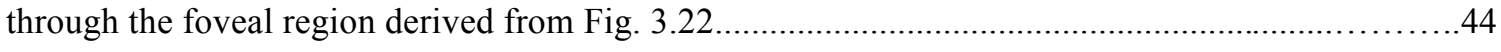

3.25. Intensity maps (average) showing the cumulative light distribution (relative) on the retina for naïve subjects free viewing while walking around the lab, hallways and outside. (Phase 5)...............44

3.26. (a) Mean retinal illumination versus eccentricity derived from Fig. 3.24. (b) Horizontal line-scan

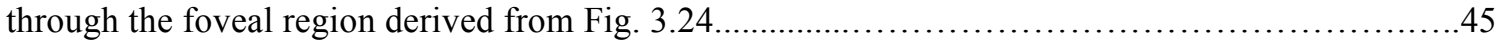

3.27. Intensity maps (average) showing the cumulative light distribution (relative) on the retina for young subjects $(<21$ years old) viewing a gray scale image. .45

3.28. (a) Mean retinal illumination versus eccentricity derived from Fig. 3.26. (b) Horizontal line-scan through the foveal region derived from Fig. 3.26. 
3.29. Intensity maps (average) showing the cumulative light distribution (relative) on the retina for subjects between the ages of 21 and 35 years old viewing a gray scale image.................................46

3.30. (a) Mean retinal illumination versus eccentricity derived from Fig. 3.28. (b) Horizontal line-scan

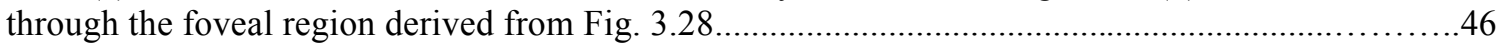

3.31. Intensity maps (average) showing the cumulative light distribution (relative) on the retina for subjects older than 35 viewing a gray scale image. 


\section{INTRODUCTION}

\subsection{Motivation and Purpose}

Age-related macular degeneration (AMD) is the third cause of blindness in the world but it is the first in industrialized countries including the United States (Resnikoff, Pascolini et al. 2004). Currently more than 1.75 million Americans are affected by the disease and the number is expected to reach about 3 million by the year 2020 (Friedman, O'Colmain et al. 2004) Although several studies have identified both genetic and environmental factors as causes for the disease, the etiology of AMD is only partly understood. Despite a concentration of several defense mechanisms in the central retina such as the absorption of light by the crystalline lens, and the presence of macular pigment and vitamin $\mathrm{E}$, it is here that macular degeneration inflicts the most damage. The high concentrations of oxygen and the presence of photosensitizers in the center of the retina, and the fact that AMD wreaks most of the damage in this region, raises the question of whether ambient light, integrated over long periods, is more concentrated in the macula.

Retinal damage due to excessive exposure to solar radiation or intense artificial sources of light has been demonstrated in a number of studies (Kirkness 1986; Gosbell, Stefanovic et al. 2006). However, while several epidemiologic studies support the role of chronic exposure to sunlight in the development of AMD (Taylor, West et al. 1992; Delcourt, Carriere et al. 2001), the acceptance that chronic exposure to less intense light sources plays a role in retinal injury is still controversial. This is due to the difficulty of assessing the spatial distribution of light in the retina by objective means. This study addressed this difficulty by developing a method, based on an eye-tracker system, that allowed measurement of the distribution of light in the retina under natural viewing conditions. The theoretical models of the eye proposed by Kooijman and Pflibsen (Kooijman, 1983; Pflibsen et al., 1988) failed to establish a relationship between the retinal area affected by AMD and the spatial distribution of light in the retina. In the present study, this issue was addressed by analyzing the relative distribution of light in the retina within $\sim 20^{\circ}$ of eccentricity from the fovea when the eye was exposed to normal, 
ambient illumination patterns. A secondary purpose was to investigate whether a relationship between age and the distribution of light in the retina exists.

\subsection{Hypothesis}

This research was predicated on preliminary data that demonstrated the feasibility of measuring light distributions on the retina, and on the observation that bright objects in the environment tend to attract our visual attention, thereby increasing the light flux in the foveal region. The central hypothesis was that, integrated over time, the flux of light falling on the retina is greater in the central macular region than in the surrounding, peripheral region. The rationale for these studies was that knowledge of the distribution of light on the retina, combined with the existing knowledge of the distribution of oxygen and photosensitizers, would allow us to predict where photooxidative damage is most likely to occur, and where antioxidants and macular pigments would be most effective. Confirmation of the hypothesis would provide a model to explain the connection between the incidence of AMD and exposure to excessive ambient light, and to explain why the eye has evolved mechanisms to limit damage by light in the central macula. It would also stress the need for protection of the retina against excessive light, and the importance of a diet that includes a sufficient intake of appropriate antioxidants. These studies are innovative in that the cumulative spatial distribution of light on the retina is unknown, and therefore its influence on the corresponding distribution of damage by AMD is only speculative

\subsection{The Human Eye.}

A basic knowledge of the structure and properties of the human eye is necessary for understanding image formation and how the eye controls light intensity at the level of the retina.

\subsubsection{The Ocular Media}

The human eye (See Fig. 1.1) is a complex organ of approximately spherical shape. It is about $2.5 \mathrm{~cm}$ wide, $2.5 \mathrm{~cm}$ deep and $2.3 \mathrm{~cm}$ tall with a marked protuberance towards the front. With a 
function similar to a camera, the eye can generate a focused image that can stimulate neural responses and produce vision. The anterior segment of the eye comprises the cornea, the iris, the pupil, the lens and a transparent jelly-like fluid (refractive index $n=1.33$ ) called the aqueous humor. The aqueous humor fills the anterior and posterior chamber and is responsible for the transport of metabolites to the avascular lens and cornea and also maintains ocular pressure.

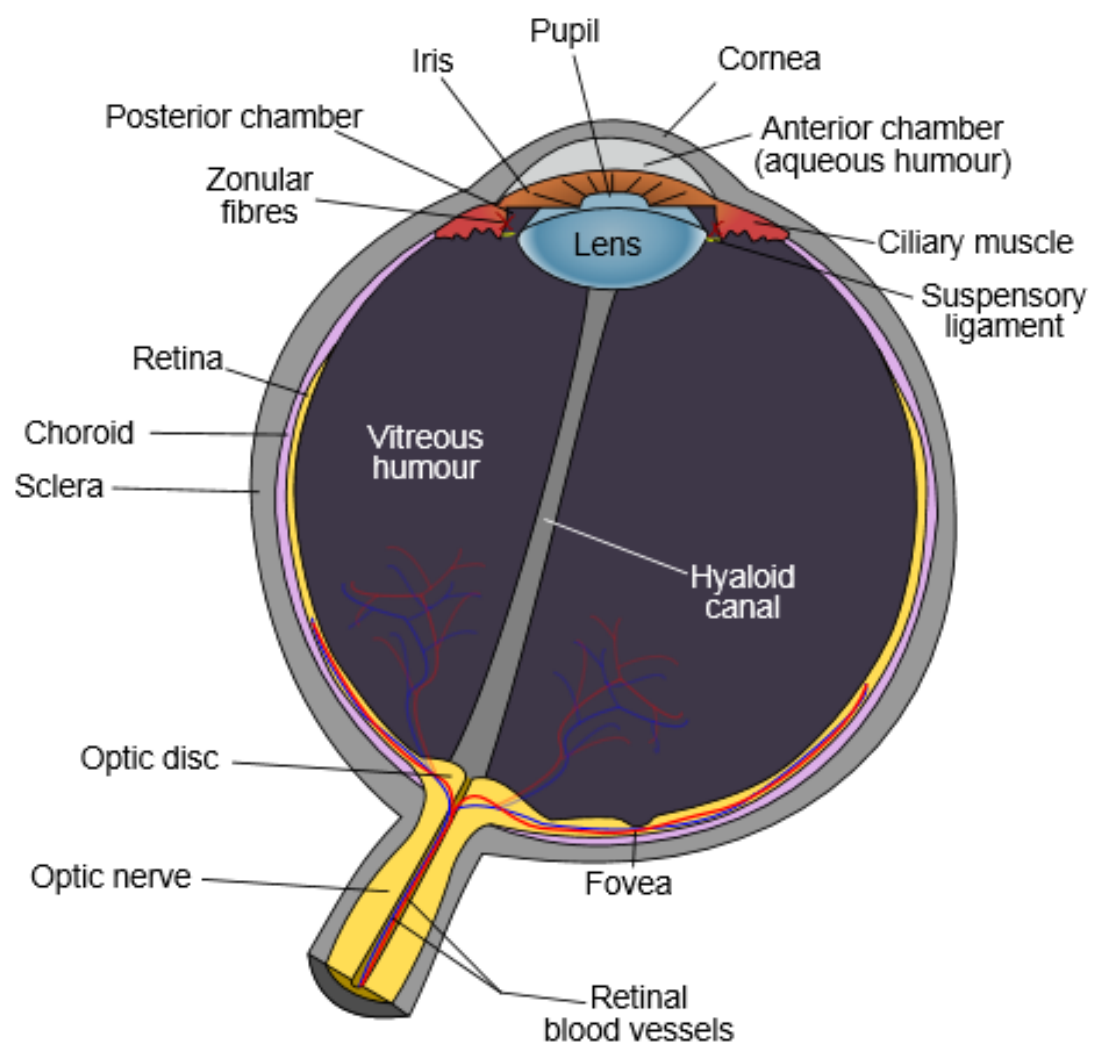

Fig. 1.1 Schematic median section of the eye seen from above. (by Rhcastilhos Wikimedia Commons)

The front part of the eye (about one-six of the surface area of the eye ball) is the cornea which is a tough, transparent layer (refractive index of 1.3375) lacking blood vessels with a radius of about 0.8 $\mathrm{cm}$ that covers the iris, the pupil and the anterior chamber. The cornea refracts light as it enters the eye and has a fixed power of refraction of around 43 diopters. The remaining five-sixths of the eye globe consists of the sclera, commonly known as the white of the eye. The sclera is a white, opaque 
protecting structure that contains collagen and elastic fiber (Forrester, Dick et al. 2002). From outer to innermost, the four layers forming the sclera are the episclera, the stroma, the lamina fusca and the endothelium (Keeley, Morin et al. 1984). The sclera meets the cornea at the corneoscleral junction or limbus (Pajoohesh-Ganji and Stepp 2005).

Behind the cornea is the pupil which is a circular aperture in the iris that allows part of the light that passes the cornea to enter the eye. The dark appearance of the pupil is attributed to the fact that most of the light that enters the eye is absorbed by the retina and other tissues in the eye's interior. As will be described later, this is an important feature for eye tracking. In 1966, Campbell and Gubisch (Campbell and Gubisch 1966) and later Howland and Donnelly (Howland and Howland 1977; Donnelly III and Roorda 2003) determined that the pupil size that offered the best lateral resolution was between 2 and $4.9 \mathrm{~mm}$ in diameter. The diameter of the pupil is controlled by the iris which is a complex, heavy-pigmented tissue that controls the amount of light that enters the eye. The dimensions of the iris vary slightly among individuals but on average it is $\sim 12 \mathrm{~mm}$ in diameter (Lefohn, Budge et al. 2003). Irises contain melanin and lipofuscin (Delori and Pflibsen 1989). In dim light, the iris dilates by means of the dilator pupillae which is the muscle responsible for dilation or mydriasis. The sphincter pupillae muscle lies near the papillary margin and is responsible for iris contraction (miosis) which normally occurs under bright conditions.

The last part of the anterior segment of the eye is the crystalline lens which is suspended inbetween the posterior chamber and the vitreous body by the suspensory ligaments or zonules. The lens is a biconvex, multilayered, ellipsoidal structure with a different radius of curvature on the anterior and posterior surfaces. The radius of the anterior surface of the lens ranges from $8 \mathrm{~mm}$ to $14 \mathrm{~mm}$, and the radius of the posterior surface ranges from $4.5 \mathrm{~mm}$ to $7.5 \mathrm{~mm}$ (Pajoohesh-Ganji and Stepp 2005). The lens body is formed by three parts: the capsule, the lens epithelium and the lens fibers. Although the lens refractive power is much less than the cornea, most of the 58 diopters refractive power of the human eye is affected by the lens through a process called accommodation. This is an increase in the dioptric power of the eye that enables the image of near objects to be focused on the retina. The 
refractive power of the lens is strongly affected by age and ranges from about 15 diopters at birth to around 2 diopters at the age of fifty (Alió, Schimchak et al. 2005; Borja, Manns et al. 2008). The index of refraction for a normal lens changes from 1.40 at the center to 1.36 on the periphery and is also greatly affected by age. (Hemenger, Garner et al. 1995; Garner, Ooi et al. 1998). Kasthurirangan and collaborators used magnetic resonance imaging to map the refractive index distribution in human eye lenses in vivo and to investigate changes with age and accommodation, and concluded that the refractive index of the central region does not change drastically with ageing, but its size increases with age and the peripheral decline in refractive index becomes steeper in older lenses (Kasthurirangan, Markwell et al. 2008). As the lens ages, proteins within it become denatured, particularly as a result of UV exposure, resulting in an increasingly yellow appearance. Thus the transmittance of the lens, particularly for shorter visible wavelengths, decreases with age

The vitreous cavity makes about two-thirds of the total volume of the eye and contains the vitreous humor which is a transparent (index of refraction $n=1.336$ ) gelatinous substance that fills the space between the retina and the lens. Unlike the situation with the lens, this transparency remains high throughout life. The vitreous humor is about $99 \%$ water and contains phagocytes which remove cellular debris from the visual field. (Nickerson 2006). One of the main functions of the vitreous humor is to keep the retina in place by pressing it against the choroid and maintaining the round shape of the eye ball. Unlike the aqueous humor which is constantly replenished, the vitreous humor stays permanently inside the vitreous cavity. With age, the vitreous degenerates and is no longer able to keep its original consistency (Meral and Bilgili 2011). When this happens, the vitreous shrinks and pulls away from the retina at the back wall of the eye. This separation of vitreous from retina is called posterior vitreous detachment. According to the National Eye Institute, vitreous detachment is common in people over age 50, and is very common after 80 (Sebag, Buckingham et al. 1992). 


\subsubsection{The Retina}

The retina is a multilayered structure that covers about $65 \%$ of the eye's interior surface. It has an area of approximately $11 \mathrm{~cm}^{2}$, and a thickness of about $250 \mu \mathrm{m}$. If accommodation is ideal, and the optic media are clear, a sharply focused, inverted image of the external scene is formed on the retina. The gaze position within the scene is imaged at the fovea, which is at the center-most point of the retina. The macula is the name given to the central region of the retina and is responsible for central and fine vision. See Fig. 1.2. The name is derived from macula lutea, meaning "yellow spot," and describes the small deposition of yellow carotenoids in the central retina. However, ophthalmologists often use the term "macula" to describe a considerably larger area, particularly in reference to macular degeneration. The central macula contains the highest concentration of cone photoreceptors. Posterior to the photoreceptor layer lies the retinal pigment epithelium (RPE). The two main functions of the RPE are photoreceptor phagocytosis (the removal of shed disks from the outer segments of photoreceptor cells) and nutrient transport. Behind the RPE is the Bruch's membrane, which is a semi-permeable transport barrier that separates the retinal pigment epithelium from the vascular layer of the retina known as the choroid.

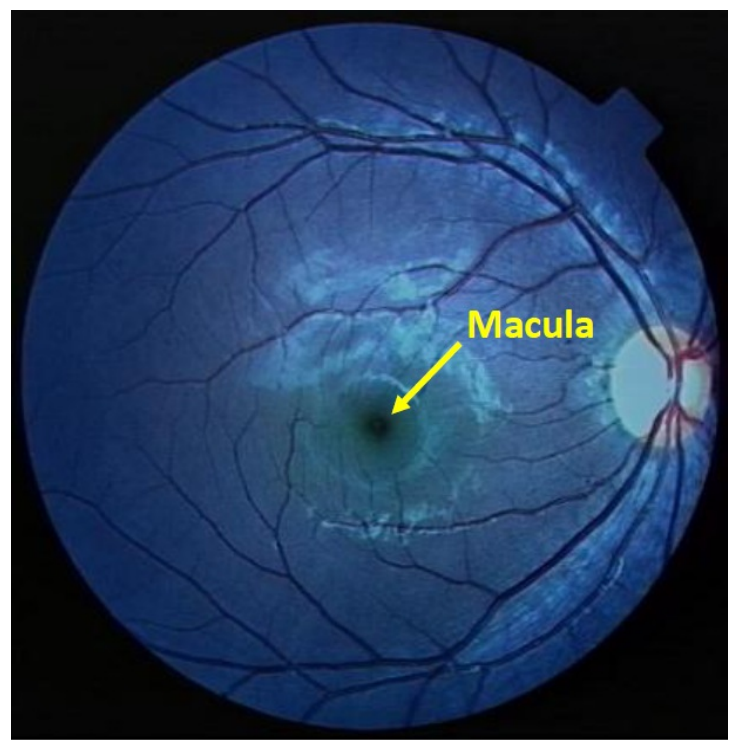

Fig. 1.2. Photographic image of the retina in blue light. The macula is the highly pigmented region at the center. 
In 1941 Polyak, described the subdivision of the retina into seven layers: retinal pigment epithelium (RPE), photoreceptor layer (outer and inner segments of rods and cones) (PL), outer nuclear layer (ON), receptor axons (RA) (also called Henle's fibers), inner nuclear layer (IN), inner plexiform layer (IP), and ganglion cell layer (GC) (Polyak 1941).

There are two types of photoreceptors in the human retina, the rods and the cones. The rods, approximately 125 million in number, abound in the peripheral regions. The cones are concentrated mainly in the fovea where the population is $\sim 190,000$ cones $/ \mathrm{mm}^{2}$ (Osterberg 1935). No cones or rods are present in the optic disk, and only cones can be seen in the centermost part of the retina. See Fig. 1.3. The rods are highly sensitive to light intensity and provide scotopic vision (vision in dim light). Cones are less sensitive to light intensity but cone vision (photopic) can distinguish different wavelengths. This is achieved in humans by having different photopigments present in different cones. Depending on their sensitivity to wavelength, the cones are subdivided into long (LWS), medium (MWS) and short (SWS) wavelength-sensitive cones. In a simplified model, a particular color sensation is generated when a stimulus results in a particular ratio of responses of the three cone types. Studies have demonstrated that in the human retina the cones are arranged in a hexagonal array with the degree of hexagonal regularity decreasing as the retinal eccentricity increases. (Gowdy and Cicerone 1998; Roorda, Metha et al. 2001)

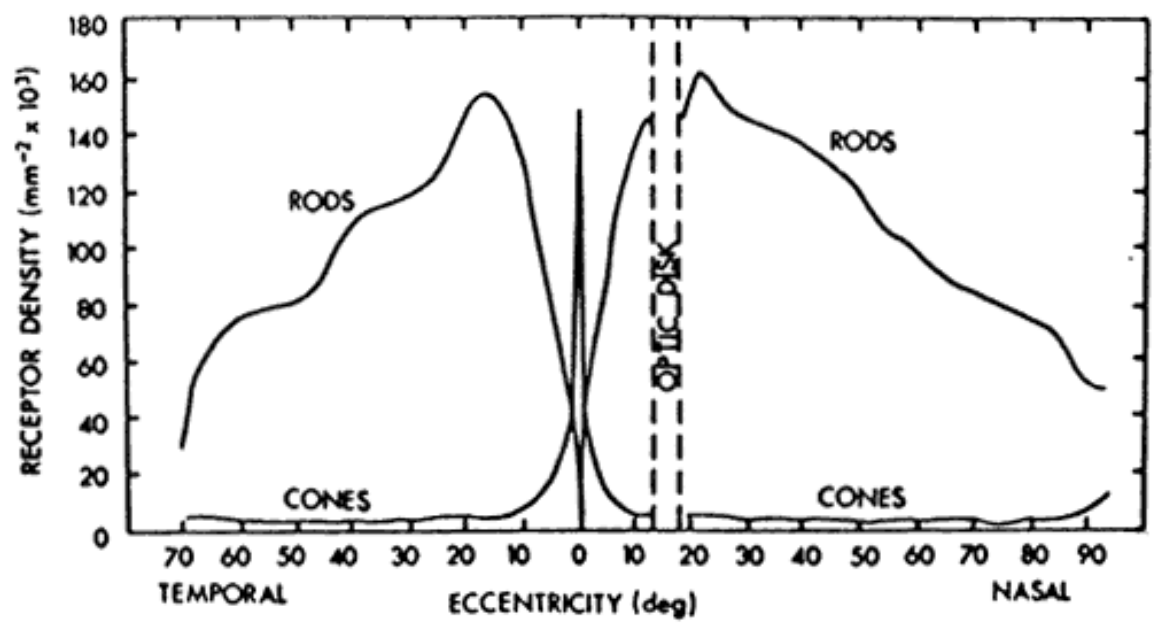

Fig. 1.3 Approximate distribution of rods and cones across the retina. The solid and dotted lines represent the cones and rods respectively (adapted from Osterberg, 1935, p.89) 
The central retina is subdivided into three regions (Polyak 1941): the fovea, the parafovea, and the perifovea. Each region subtends an outer diameter of approximately 1.5, 2.5, and $5.5 \mathrm{~mm}$ respectively. The fovea is a shallow depression within the yellow spot (macula lutea). The centermost portion of the fovea is called the foveola which lacks blood vessels, subtends a visual angle of about $1.4^{\circ}$ degrees and contains cones only. See Fig. 1.4.
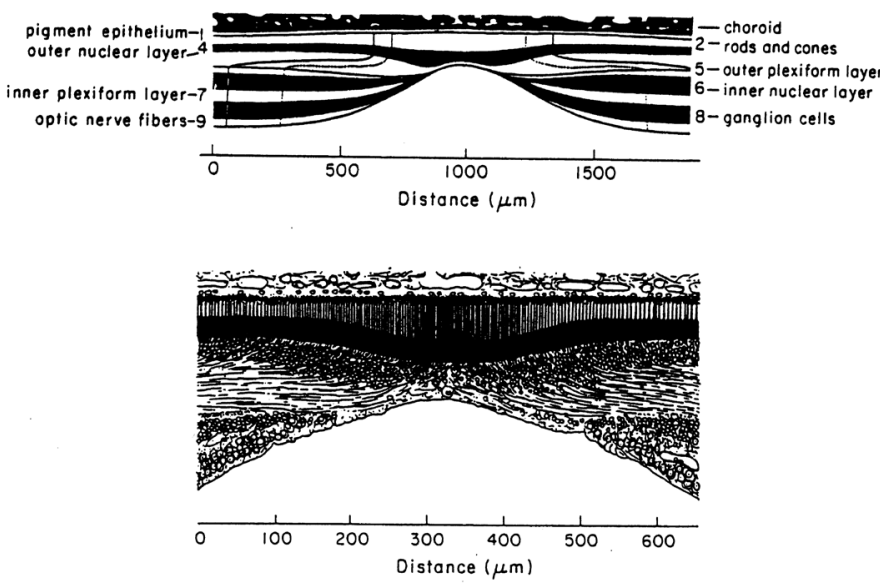

Fig. 1.4. Structure of the human fovea (Polyak, 1941, p.69).

\subsubsection{Reflectance in the human eye}

In order to understand retinal illuminance, it is necessary to be aware of factors that may modify it. One of these is reflectance. Light that passes through the ocular media is first reflected by the inner limiting membrane (ILM). Due to the small change in the index of refraction, less than $1 \%$ of the light is reflected in this interface (Knighton, Jacobson et al. 1989). Van de Kraats et al. reported a low $(0.26 \%)$ reflectance at the ILM with lower specular reflections at the foveal location than at extra-foveal locations. This characteristic was more visible in young subjects (Van de Kraats, Berendschot et al. 1996). Because the reflectance is so low, its effect on retinal illuminance can justifiably be ignored. After crossing the photoreceptor layer, light is successively reflected by the RPE, choroid, and finally by the sclera. The latter is responsible for approximately $10 \%$ of the total fundus reflectance (Van Norren and Tiemeijer 1986). Most of the reflected light appears to be the result of multiple scattering by choroidal melanin. 


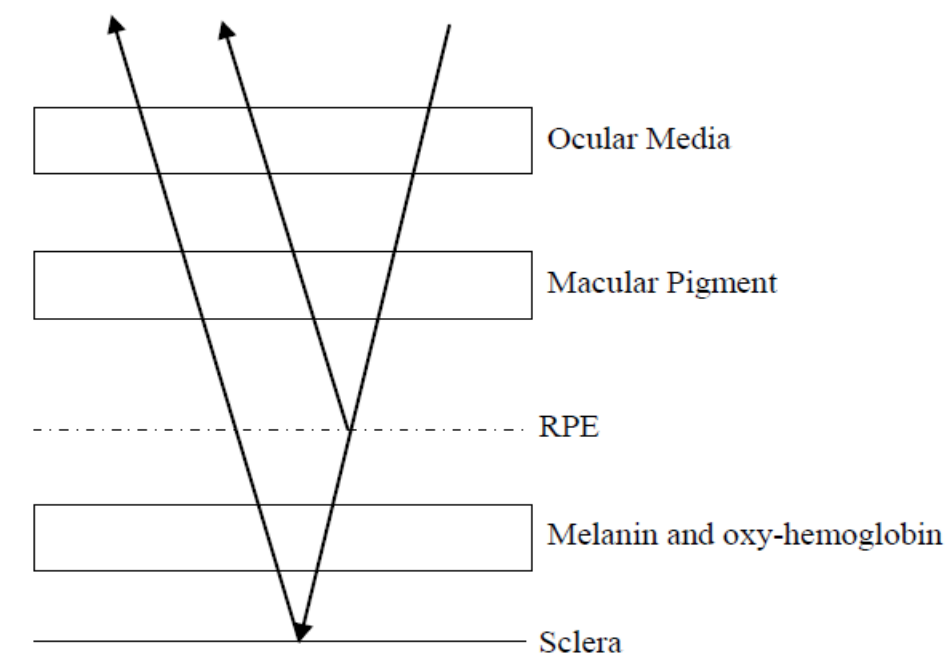

Fig. 1.5. Schema of the model of reflectances and absorptions in the human eye. (Van NorrenTiemeijer, 1986, p. 316).

\subsubsection{Spectral Reflection and Absorption in the Human Eye}

Interpretation of the spectral reflectance of the ocular fundus entails a detailed knowledge of the absorption characteristics of the different pigments in the light path as well as the reflectance of the retinal layers. Brindley and Willmer made the first measurements in 1952. They measured the spectrum of light reflected from the macula and compared this with light reflected from a point $9.5^{\circ}$ away. The difference spectrum between these two locations was similar to that of the macular pigment. In 1986, Van Norren and Tiemeijer (Van Norren and Tiemeijer 1986) proposed a simplified model of reflection and absorption in the human eye with four ocular pigments (lens pigment, melanin, oxy-hemoglobin, and macular pigment) as the primary determinants of the reflectance characteristic of the bleached fundus, and reflectance at two layers (RPE, and sclera). See Fig. 1.5. Bleaching the photopigments of the cones and rods with relatively high intensity light largely eliminates their capacity to absorb light and their contribution to the reflectance properties of the fundus. Later in 1989, Delori and Pflibsen incorporated a light-scattering component in the choroid, and included several constant parameters corresponding to specific anatomical layers of the retina. (Delori and Pflibsen 1989). Although all reflectance spectra were obtained with bleached visual photopigments, it is obvious that any unbleached photopigment still affects the reflectance spectrum 
(Delori, Goger et al. 2001; Bour, Koo et al. 2002). While nearly $100 \%$ of cone photopigments can easily be bleached, it is difficult to bleach more than $\sim 70 \%$ of rod photopigment. Fig. 1.6 shows the density spectra of the relevant absorbing pigments in the human eye.

The ocular media, principally the lens, is the first in absorbing both the incident and the reflected light. In young subjects, the absorption by the ocular media is constant above $650 \mathrm{~nm}$ and increases drastically from 560 to $400 \mathrm{~nm}$. (Van Norren and Vos 1974). The absorption by the media markedly increases with age. (Pokorny, Smith et al. 1987; Delori and Burns 1996). In the present study, only the relative retinal illuminance will be presented, and this will be unaffected by absorption in the lens. (Absolute retinal illuminance would, of course, be affected, and would be wavelength dependent.)

Melanin is located principally in the retinal pigment epithelium (RPE) and choroid. It has a high absorption over the whole visible spectrum, and it is the predominant absorbing pigment for wavelengths between 650 and 750nm (Hunold and Malessa 1974). Van Norren and Tiemeijer found about $30 \%$ more melanin density in foveal locations than in peripheral locations with a marked racial dependence (Van Norren and Tiemeijer 1986).

Absorption of light by blood takes place mainly in the choroid. The absorption spectrum of the blood in the retina is dominated by oxy-hemoglobin, which has a high absorption at blue and green wavelengths. Van Norren and Tiemeijer reported a hemoglobin density of 0.18 density units (DU) at $500 \mathrm{~nm}$ with no significant difference between the fovea and perifovea except for the optic disk, which has a density of 0.28 DU (Van Norren and Tiemeijer 1986). 


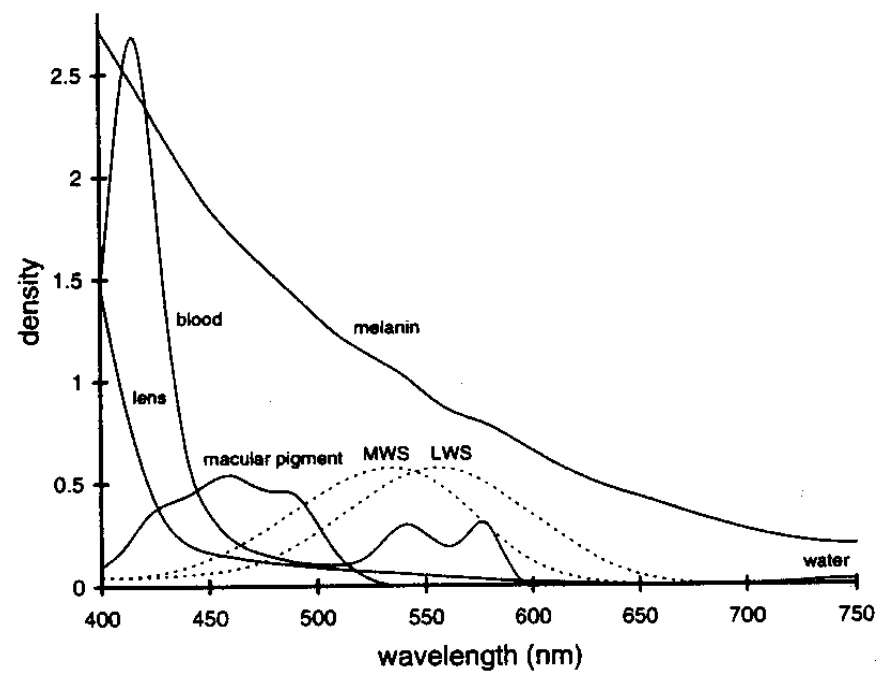

Fig. 1.6. Absorption spectra of blood, melanin, macular pigment, lens, and water in the eye for a single pass. (Van de Kraats, Berendschot et al. 1996)

The macular pigment absorbs the shorter wavelengths, 400 to $500 \mathrm{~nm}$ and is much more highly concentrated in and around the fovea. The yellow macular pigment is located primarily in the photoreceptor axons and inner plexiform layers of the macula. The spatial distribution of the macular pigment is similar to that of the cones. It reaches a peak density within the foveola and declines to a low, approximately constant level within $4^{\circ}$ eccentricity (Snodderly, Auran et al. 1984). In 1945, Wald found that the macular pigment absorbed wavelengths between 430 and $490 \mathrm{~nm}$ with an absorption peak (of about $60 \%$ ) at $465 \mathrm{~nm}$ (Wald 1945). He also noted that the shape of the absorption curve of the macular pigment was consistent with its being a carotenoid, possibly the xanthophyll lutein. (Xanthophylls represent a class of carotenoids characterized by the presence of oxygen in their molecular structure.) By cutting the retina into six annuli concentric with the fovea and performing HPLC analysis of the samples, Bone and Landrum. (Bone, Landrum et al. 1988) were able to show how the distribution of lutein and zeaxanthin varies with eccentricity. See Fig. 1.7. Later, they found that the retinal zeaxanthin is itself composed of all three possible stereoisomers, $3 \mathrm{R}, 3^{\prime} \mathrm{R}-$ zeaxanthin, 3R, 3'S-zeaxanthin (meso-zeaxanthin), and 3S, 3'S-zeaxanthin (Bone, Landrum et al. 1997). 


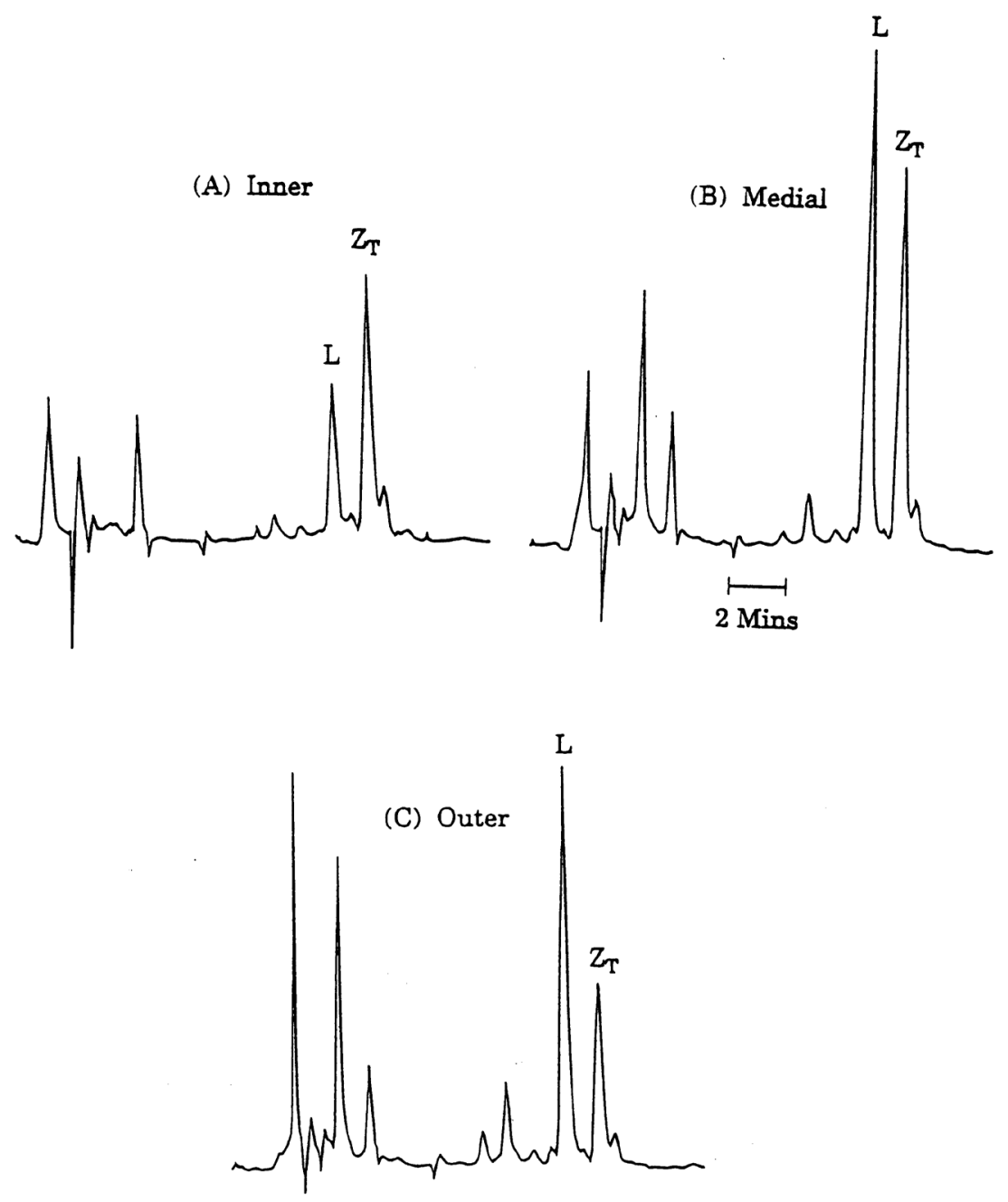

Fig. 1.7 Representative chromatograms of photopigments extracted from three different regions of retinal tissue. (A) $7.1 \mathrm{~mm}^{2}$ disk centered on the fovea. (B) $93-\mathrm{mm}^{2}$ annulus. (C) $343 \mathrm{~mm}^{2}$ annulus. $\mathrm{L}=$ Lutein, $\mathrm{Z}=$ Zeaxanthin (Bone, Landrum et al. 1988, p. 539).

Using a modified retinal camera, Bone et al. (Bone, Brener, Gibert 2007) developed an objective method to determine the optical density distributions of macular pigment, photopigments and melanin in young subjects $(<25$ years). Their results confirmed that the distribution of both cone photopigments and macular pigment peaked at the fovea, whereas rod photopigment dipped. Melanin was found to be more spread over the central retina. See Fig. 1.8. 

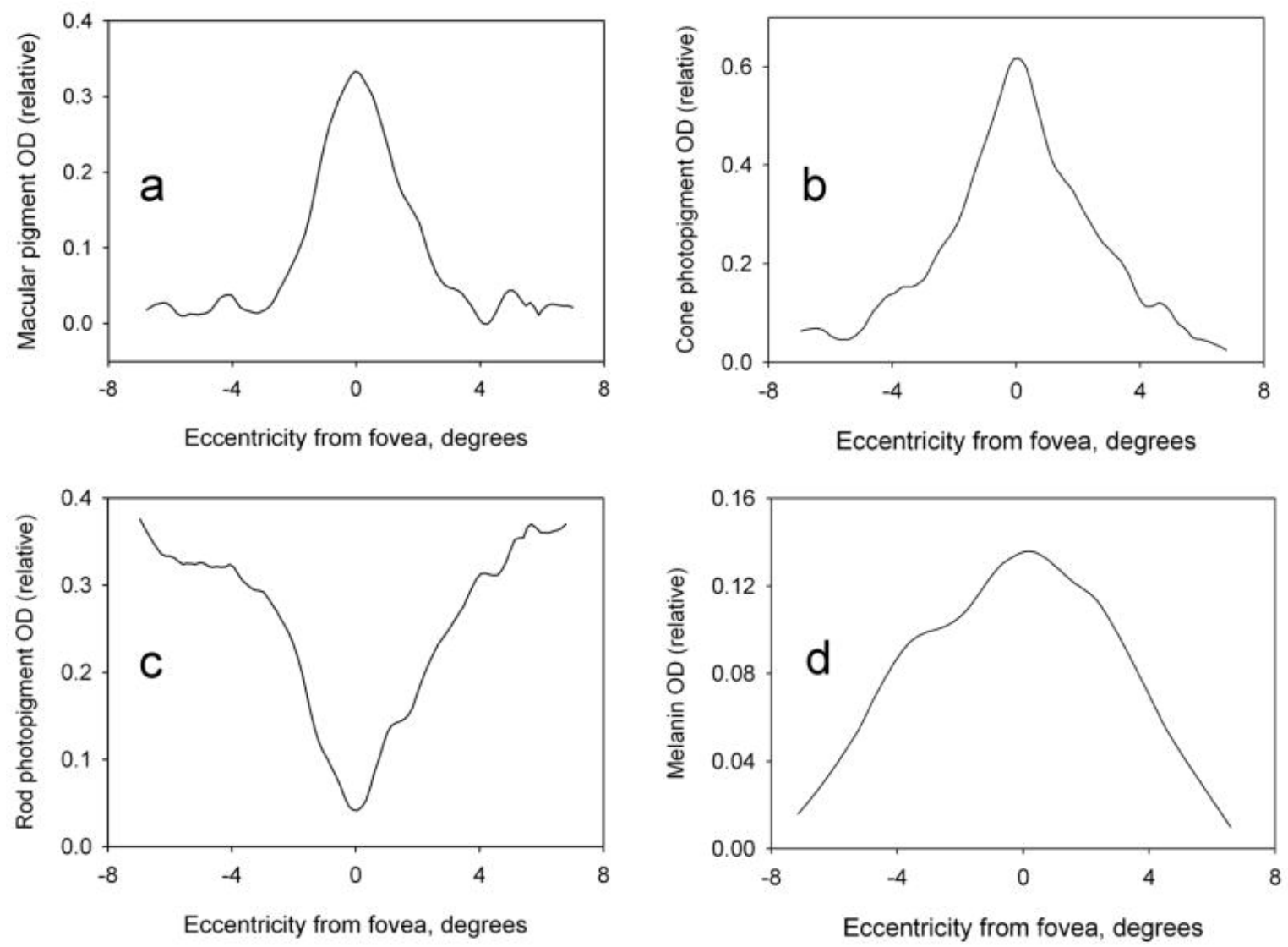

Fig. 1.8. Relative optical density distributions at $460 \mathrm{~nm}$ of (a) macular pigment, (b) cone photopigment, (c) rod photopigment, and d) melanin along a horizontal meridian through the fovea. (Bone, Brener, Gibert., 2007, p. 3275).

Because its absorption peaks at $\sim 460 \mathrm{~nm}$, the macular pigment may improve visual acuity by reducing the effects of chromatic aberration and atmospheric light scatter (Reading and Weale 1974). Recent studies suggest that the macular pigment protects the central retina from the damaging effects of blue light (Kirschfeld 1982; Bone and Landrum 1984; Landrum, Bone et al. 1996). Ultraviolet light ranging from $300 \mathrm{~nm}$ to $400 \mathrm{~nm}$ is almost completely absorbed by the crystalline lens (Weale 1988) and consequently does not represent a threat to the retina. Blue light can induce the formation of reactive radicals, triplet excited states, superoxide, and singlet oxygen in the choriocapillaris, Bruch's membrane, and RPE, which leads to the photo-degradation of the photoreceptors in the macula (Gottsch, Pou et al. 1990). Studies have demonstrated that the development of this mechanism is reduced in individuals with high concentrations of macular pigment (Pokorny, Smith et al. 1987). Li et al used electron paramagnetic resonance spectroscopy to measure light-induced singlet oxygen 
generation in postmortem human macula and RPE, and detected singlet oxygen production in the RPE and choroid but not in the macular tissue. The study also showed that a mixture of meso-zeaxanthin, zeaxanthin, and lutein in a ratio of 1:1:1 has the ability to quench more singlet oxygen than do the individual carotenoids at the same concentration (Li, Ahmed et al. 2010).

With age, the retina fails to fully dispose of waste products, and yellowish focal deposits, called drusen, appear between the RPE and Bruch's membrane. Drusen diameters range from small $(<63 \mu \mathrm{m})$ to large $(>124 \mu \mathrm{m})$ and are categorized as hard or soft depending on the form of their margins. The presence of hard drusen is normal in people over the age of 40 , but the presence of larger and more numerous drusen in the macula is a common early sign of AMD (Curcio, Millican et al. 1993).

While all of the retinal pigments will attenuate the light passing through them in a wavelength-dependent manner, they will have no effect on the distribution of light incident upon the retina, which is the focus of this study.

\subsection{Age-Related Macular Degeneration}

Age-related macular degeneration (AMD) is the leading cause of severe vision loss among the elderly in many Western countries. Currently there are an estimated 25 to 30 million people with AMD, and this number is expected to triple within the next 25 years (Hyman 1992; Rovner and Casten 2002; Friedman, O'Colmain et al. 2004). Studies have shown that AMD substantially reduces the quality of life of affected people and is a risk factor for depression among the elderly (Hayman, Kerse et al. 2007; Casten and Rovner 2008).

\subsubsection{Classification}

The Age-Related Eye Disease Study (AREDS) (Age-Related Eye Disease Research Study Group 2000) classified AMD by the size and extent of drusen, the presence of geographic atrophy, and choroidal neovascularization. Early AMD is characterized by the presence of a few (less than 20) 
medium size drusen, or by RPE hyperpigmentation. At this stage, the visual loss is generally mild or imperceptible. Intermediate AMD is characterized by at least one large druse, numerous medium size drusen, or geographic atrophy in the peripheral region of the macula. Advanced AMD is classified as dry (non-neovascular) and wet (neovascular) (Bird, Bressler et al. 1995). The dry form of advanced AMD results from atrophy of the RPE which extends to the center of the macula and causes impaired vision through loss of photoreceptors. (Dorey, Wu et al. 1989; Curcio, Millican et al. 1993). Advanced neovascular (or wet) AMD is characterized by choroidal neovascularization. The blood vessels developed in this stage leak into the retina, causing distortion or loss of central vision. These abnormal blood vessels eventually scar causing a rapid and permanent loss of central vision (Jager, Mieler et al. 2008). Although wet AMD represents less than $15 \%$ of AMD prevalence in the United States, this form of AMD is responsible for more than $80 \%$ of debilitating visual loss in AMD (Friedman, O'Colmain et al. 2004).

\subsubsection{Risk Factors}

Risk factors for AMD include genetic and environmental factors such as family history, age, gender, race, cigarette smoking, alcohol, and high blood pressure. Among the environmental risk factors, oxidative processes appear to play a common role. Many studies have identified age as the most important risk factor for AMD. Approximately $10 \%$ of patients 65 to 75 years of age show signs of macular degeneration. The prevalence increases to about $30 \%$ in patients over 90 years old (Evans 2001). Smoking is a recognized environmental risk factor. Several studies suggest that the incidence of wet macular degeneration is higher in active smokers compared with nonsmokers. Cigarette smoke contains several oxidants that reduce plasma antioxidant protection and choroidal blood flow. (Klein, Klein et al. 1993; McCarty, Mukesh et al. 2001). Lower levels of macular pigment density have been demonstrated to be associated with AMD, retinal damage, and risk factors for AMD (HaegerstromPortnoy 1988; Snodderly 1995). Studies suggest the possibility of reducing the risk of AMD by increasing the macular pigment optical density (MPOD) (Bone, Landrum et al. 2001). Vitamin C and E are two other antioxidants essential for protection against disease progression and degenerative 
disorders caused by oxidative stress (Eye Disease Case-Control Study Group 1993). In a systematic review and meta-analysis study performed by Chakravarthy and collaborators, smoking, previous cataract surgery, and a family history of AMD showed strong associations with late AMD while higher body mass index, history of cardiovascular disease, hypertension, and higher plasma fibrinogen were identified as moderate risk factors. (Chakravarthy, Wong, et al. 2010). Gender, ethnicity, and iris color has been found to be weak risk factors (Hammond Jr, Curran-Celentano et al. 1996; Thornton, Edwards et al. 2005).

\subsubsection{Light and AMD}

Evidence that light may be implicated in AMD is based partly on epidemiology studies. In one study involving watermen in Chesapeake Bay, ocular exposure to sunlight over a period of 20 years was deemed a probable risk factor for AMD (Taylor, West et al. 1992). A similar association between visible light exposure and AMD was reported in the Beaver Dam Eye Study. (Cruickshanks, Klein et al. 1993). In this study, sunlight exposure was estimated from the amount of time that subjects spent outdoors and the use of sunglasses and hats with brims. In a study carried with 1300 people on the Croatian island of Rab, it was found that there was an $18 \%$ incidence of AMD on fisherman and farmers compared with $2.5 \%$ for town-dwellers who were presumably less exposed to the high levels of solar radiation (Vojniković, Njirić et al. 2007). In contrast, data from three Australian studies have produced mixed results. In a cross-sectional, population-based study, blue iris color (a possible marker of higher retinal light exposure) was significantly associated with increased risk of late AMD (Mitchell, Smith et al. 1998). However, an increased risk of late AMD was associated with both high and low sun sensitivity of the skin, presumably a marker of the time a person spends exposed to intense sunlight. In a second, case-control study, control subjects had a roughly $20 \%$ greater median annual ocular sun exposure than AMD cases (Darzins, Mitchell et al. 1997). In a third study, the mean annual ocular sun exposure over either a lifetime or the previous 20 years was greater for people with AMD than for those without, but not significantly so (McCarty, Mukesh et al. 2001). In a French study, in which residential history was used to estimate individual 
annual sunlight exposure, such exposure was not significantly related to increased risk of AMD (Delcourt, Carriere et al. 2001). However, frequent use of sunglasses was significantly associated with a reduced risk of soft drusen. Therefore the association between AMD and light exposure is not as well established as the association between AMD and smoking though this is likely owing to the difficulty of quantifying a person's retinal exposure to light and the presence of confounding factors. Adding to the difficulty, either total cumulative light exposure, or cumulative exposure to more intense light above some threshold intensity, could conceivably be the most important factor in the incidence of AMD. Margarian et al. suggested that blue light might have a role in the prevalence of AMD (Margrain, Boulton et al. 2004). A comprehensive literature review and meta-analysis of 14 publications performed by Sui and collaborators showed that individuals with more sunlight exposure are at a significantly higher risk of AMD (Sui, Liu et al.). At the moment, the distributions in the retina of light filters (macular pigments), photosensitizers, oxygen and the cells affected by AMD are well known. However, the current knowledge of the distribution of light across the retina is based only on analysis of theoretical models of the eye.

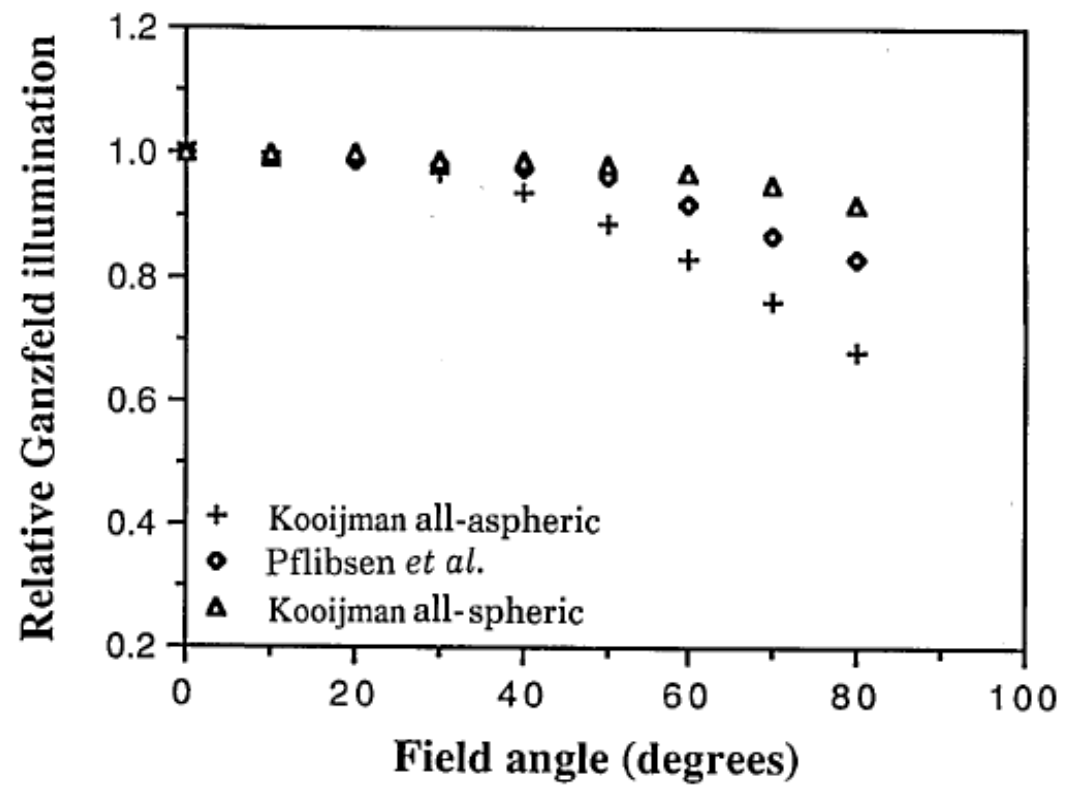

Fig. 1.9. Relative Ganzfeld illumination of the retina as a function of field angle without considering media extinction. Results from Kooijman's all-aspheric, Pflibsen, and Kooijman's all-spherical (Adapted from Pflibsen et al, 1988) 
Fig. 1.9 shows the results obtained from a model developed by Kooijman and later updated by Pflibsen. The relative Ganzfeld illumination (spatially and temporally uniform visual field) of the retina as a function the visual angle for a wide-angle model (WAM) of the eye with spherical surfaces predicted a small decrease in illumination with eccentricity. (Kooijman 1983). A larger decrease in retinal illumination with eccentricity was predicted when aspheric corneal, lens, and retinal surfaces were used, and the extinction of the lens was included. (Kooijman 1983; Pflibsen, Pomerantzeff et al. 1988). However both studies showed a relatively uniform distribution of light on the retina up to $\sim 50^{\circ}$ of eccentricity, a result that failed to establish a relationship between retinal light distribution and the prevalence of AMD in the center of the retina. One of the purposes of the present study was to examine this potential relationship by developing an experimental method in which the light distribution on the retina, up to $\sim 20$ degrees of eccentricity, was assessed under natural viewing conditions. 


\section{METHODOLOGY}

\subsection{Preliminary Studies.}

This research was based on a preliminary pilot study that demonstrated the possibility of measuring the distribution of light in the human retina, and on the observation that bright objects in the environment tend to attract visual attention, thereby increasing the light flux in the macular region. Preliminary studies were conducted with the head-mounted eye-tracker provided by SensoMotoric Instruments (SMI) shown in Fig. 2.1.

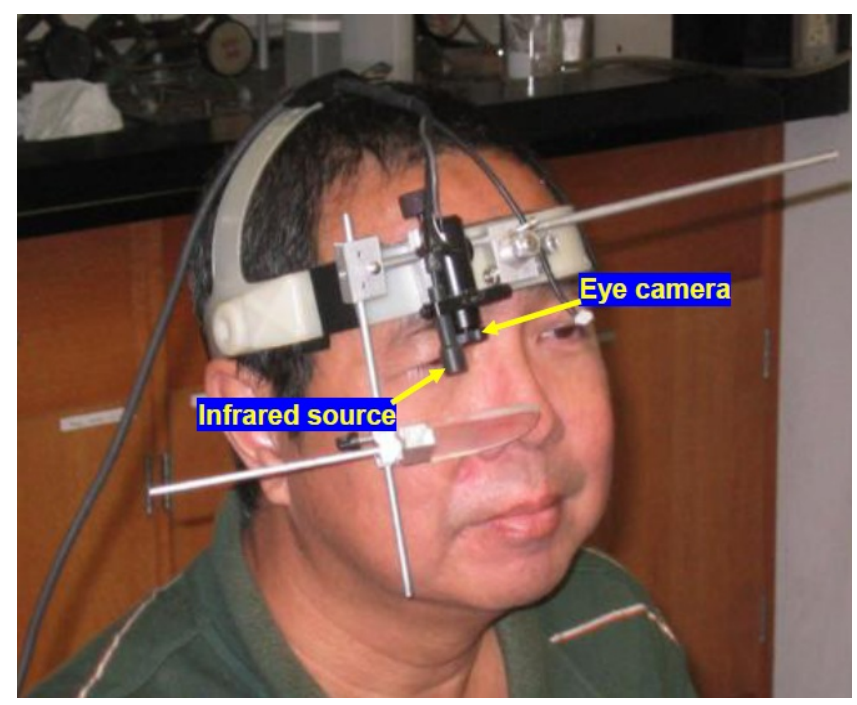

Fig. 2.1. Modified eye-tracker (SensoMotoric Instruments SMI ${ }^{\circledR}$ ) used for preliminary studies.

Briefly stated, a subject, wearing the eye-tracker, viewed an image displayed on a 16 ", computer monitor. The monitor was $\sim 3$ ' from the subject's face. Head position was maintained with a mouth bite that immobilized the subject's upper jaw. Based on the subject's gaze position and the distribution of light on the monitor screen, the corresponding light distribution on the retina in and around the fovea was calculated. This was done for each gaze position, and the cumulative light distribution on the retina was determined by simply adding the individual distributions. Fig. $2.2 \mathrm{~b}$ is a pictorial representation of the relative retinal illuminance when a subject was instructed to inspect a simple image (Fig. 2.2a) displayed on the monitor. The center of the image corresponds to the fovea. 
The data was quantified by running a horizontal line-scan through the point corresponding to the fovea. Fig. 2.2c is such a scan and shows a peak in the retinal light distribution that is centered on the fovea.

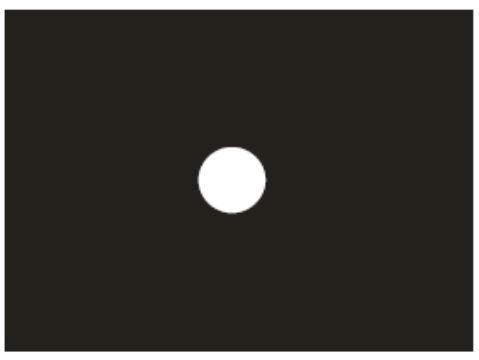

(a)

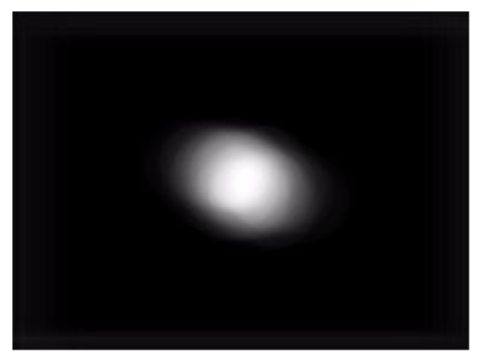

(b)

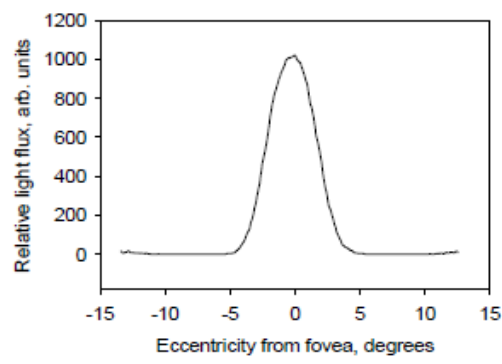

(c)

Fig. 2.2. Simple test used in the preliminary study: (a) visual stimulus. (b) pictorial representation of the retinal illuminance when the subject fixated on the white region in (a) (c) horizontal line-scan through (b) showing a peak illuminance at the center of the retina.

Fig. $2.3 \mathrm{~b}$ is a grayscale pictorial representation of the cumulative light distribution on a $\sim 18^{\circ} \times 12^{\circ}$ area of the retina for a subject who spent 1 minute viewing the image shown in Fig. 2.3a. The bright area again represents the fovea. (It should be at the center but is displaced slightly downwards as a result of imprecise calibration or movements of the head mount after calibration.

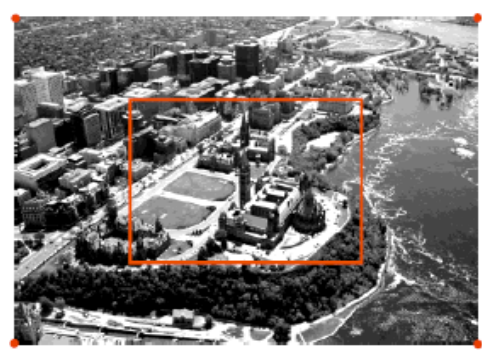

(a)

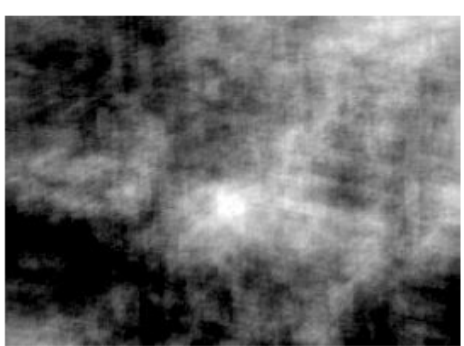

(b)

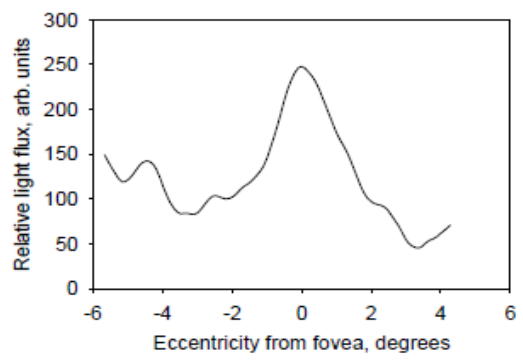

(c)

Fig. 2.3. Test used in preliminary studies. (a) Image freely viewed by subject for 1 minute (b) Resulting retinal light distribution (c) Vertical line scan through center of (b)

A vertical line scan through the foveal region is shown in Fig. 2.3c. The implication is that this subject spent more time fixating on bright objects on the monitor screen compared with darker objects and therefore the retinal illuminance (relative) peaked in the foveal region. These preliminary experiments demonstrated the feasibility of using a modified eye-tracker to assess the distribution of light in the retina. 


\subsection{Eye Tracking System}

The eye tracking system used in the main study (shown in Fig. 2.4) was provided by Arrington Research ${ }^{\circledR}$ and consisted of a light-weight spectacle frame on which were mounted a scene camera, a monocular, infra-red-sensitive eye-tracking camera, and an infrared LED illuminator (IRED). The eye-tracker was connected to a computer by a long cable.

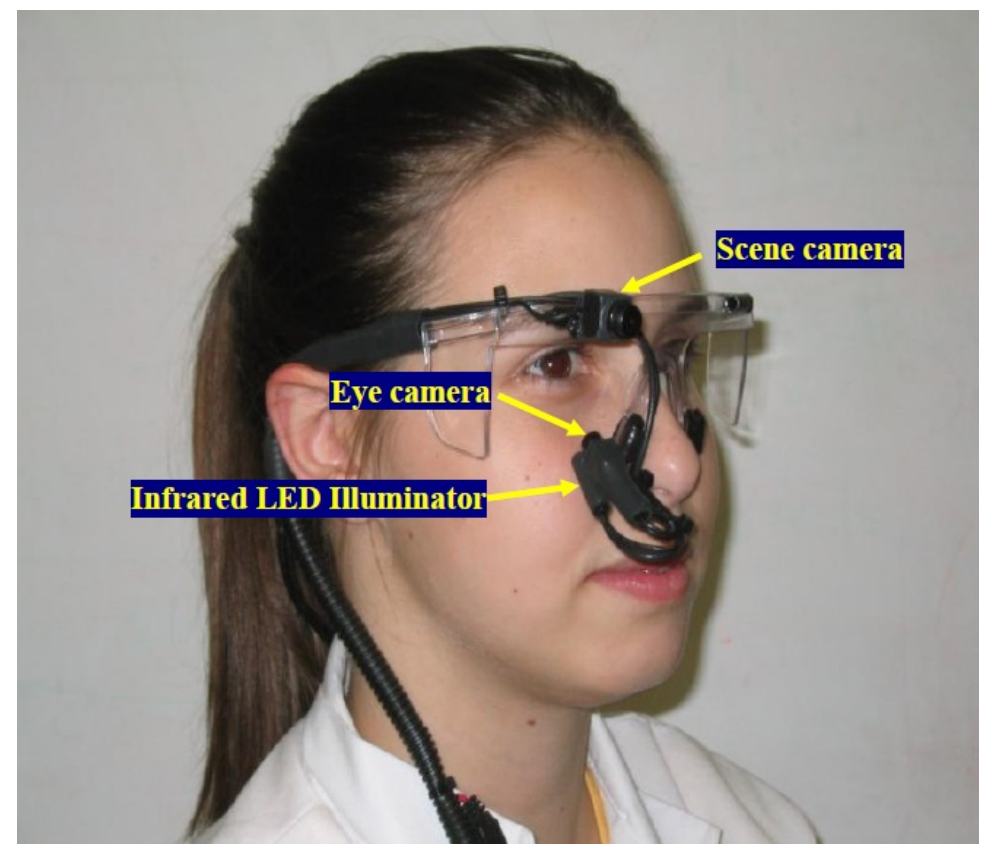

Fig. 2.4. Eye-Tracking system provided by Arrington Research ${ }^{\circledR}$

The system provided simultaneous scene video recording, and eye movement and pupil diameter capture (EyeTracker 2005). The IRED illuminated the eye, and also provided a corneal "glint," (corneal reflection) which aided in determining gaze direction. A potential risk factor that could be associated with this study was the intensity of near-infrared radiation reaching the retina, which is a function of the power emitted by the IRED, the area over which that energy is spread, and the uniformity of the illumination pattern. Since the human retina does not react to wavelengths in the near-infrared zone, the operator could not rely on the subject's reaction to illumination. In this eye tracking-system, the IRED had an intensity below $10 \mathrm{~mW} / \mathrm{cm}^{2}$, which is the limit for corneal exposure over prolonged periods of time (Clarkson 1989; Sliney, Aron-Rosa et al. 2005). 
In "dark pupil mode," which was the method used throughout the study, the pupil acted as an infrared black body. See Fig. 2.5. The video signal from the eye camera was digitized by a video capture card and transferred to the computer for further analysis. The computer took the digitized image and applied image segmentation algorithms to locate the area of the pupil and the location of the corneal glint. A mapping function transformed the eye position signals into the subject's gaze coordinates. To determine the gaze position, the eye-tracker system measures the displacement (magnitude and direction) from the center of the pupil to the center of the corneal glint.

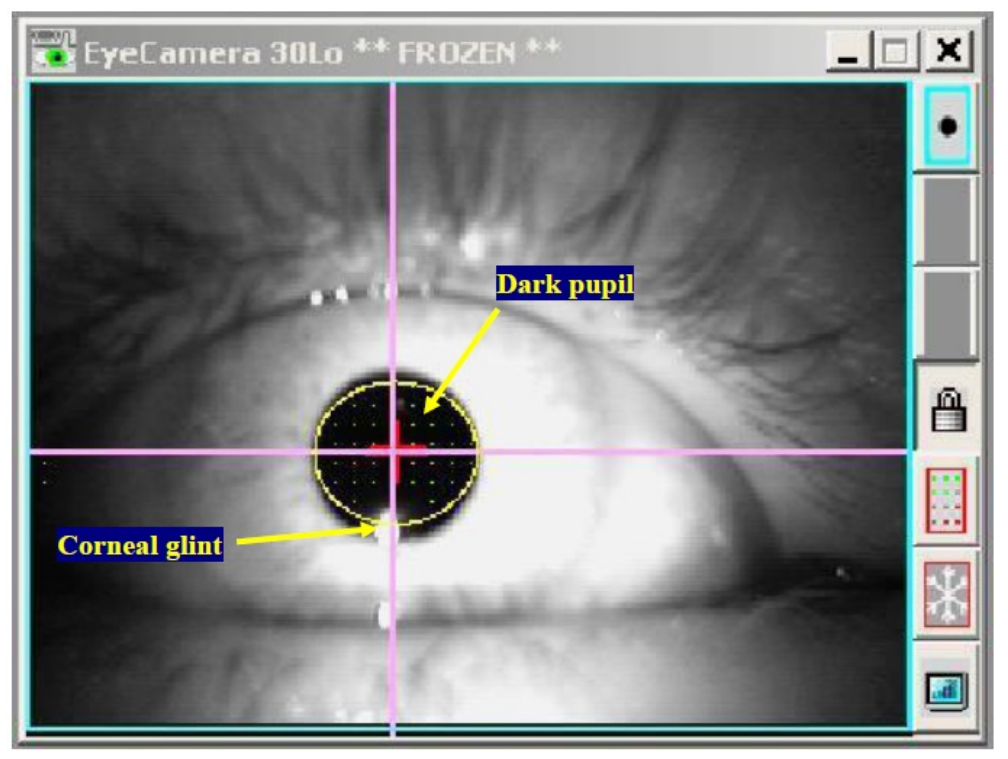

Fig. 2.5. Video frame recorded by the eye-camera showing the dark pupil and the corneal glint. The system measures the vector length between the center of the pupil (shown in red) and the center of the corneal glint.

Additionally, the height and width $( \pm 0.3 \mathrm{~mm})$ of the pupil were recorded, from which the pupil area was subsequently calculated. The system also provided for automatic blink and saccade detection and suppression. The scene camera had an 8-bit CCD element with a built-in gamma correction and electronic aperture that limited the maximum pixel value to 255 . Its resolution was set to 320 by 240 pixels. Its purpose for this study was to act as a semi-quantitative imaging photometer, with the individual pixel values (0-255) providing an estimate of the relative light intensity at the corresponding points in the visual field. For some of the experiments, the subject was seated 1.5 meters from a large projection screen. The subject's head was immobilized by a head restraint system. 
The restraint system consisted of forehead and chin supports and a head strap. The positioning of the subject with respect to the screen followed the guidelines supplied by Arrington Research ${ }^{\circledR}$ (EyeTracker 2005). For example, whenever the projection screen was used, it was positioned so that when the subject was looking straight ahead, the gaze position was centered horizontally, and 2/3 of the way up vertically, measured from the bottom of the screen. The eye camera was placed $45^{\circ}$ below the line of sight of the subject. The IRED was positioned so that it appeared at the 11 o'clock position when the subject was looking at the eye camera lens. The eye camera was then moved sideways, such that the IRED was centered along the optical axis of the eye when looking at the center of the display. The lens of the eye camera was adjusted so that the pupil was centered at the eye camera window (shown on the computer monitor). The system was tightly attached to the subject's head to avoid movement between the head and the eye camera.

\subsection{Calibration}

A standard calibration of the system was performed for each subject prior to every experiment as well as between trials. In Phase 1, the calibration was performed with the automatic (head fixed) feature of the eye tracker system. The calibration mode was set to "Tunneling Motion". In this mode, the subject was instructed to fixate at the center of the screen. After a few seconds a green rectangular frame appeared at the location of one of the 16 calibration points. While the subject looked directly at the center of the stimulus the rectangular frame gradually shrunk capturing the subject's visual attention. This process continued for the remaining 15 calibration points. For Phases 2 and 3 , the computer monitor displayed the live image being captured by the scene camera with an overlaid grid of small, circular calibration targets, one of which (the one to be calibrated) appeared in blue. The monitor also displayed the apparent gaze position as another small green circle. A laser pointer was directed at the projection screen and positioned so that the monitor image of the laser spot was centered on the colored target. The subject was asked to maintain fixation on the spot on the projection screen while the gaze position was captured by depressing the appropriate key on a 
computer keyboard. This caused the next circular target in the "EyeSpace" window to appear white, and the procedure was repeated for a total of 16 targets. For Phases 4 and 5 the calibration was completed by the "snap-and-increment" method provided by the eye tracker system in which the calibration was performed relative to the pixels of the CCD array of the scene camera. The subjects were asked to sit comfortably and to remain still for the duration of the calibration process. A wand with a red tip was positioned in front of the subject so that the tip appeared inside the "GazeSpace" window within the active calibration circle. Keeping their head still, the subjects moved their eyes to fixate on the tip of the wand. When the operator was sure that the subject was looking at the correct point, the operator captured the gaze position by depressing the appropriate key on the computer keyboard. The process was repeated for all 16 calibration points.

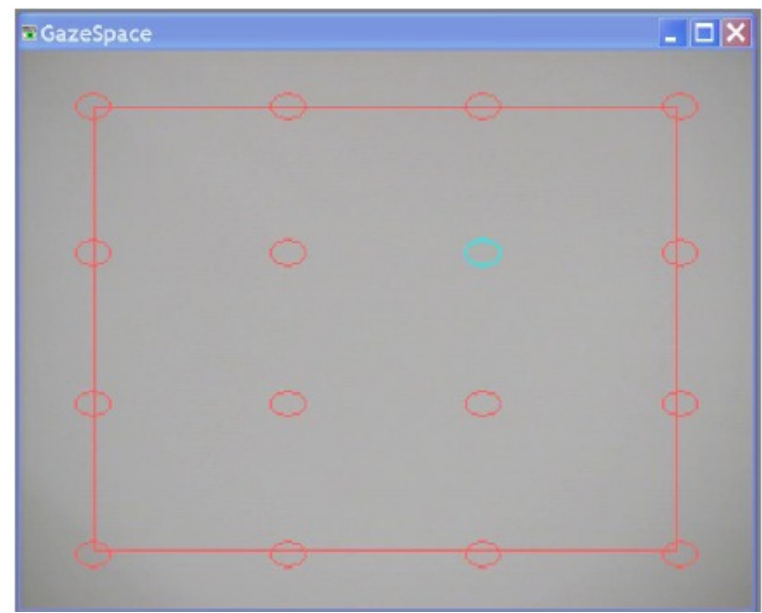

(a)

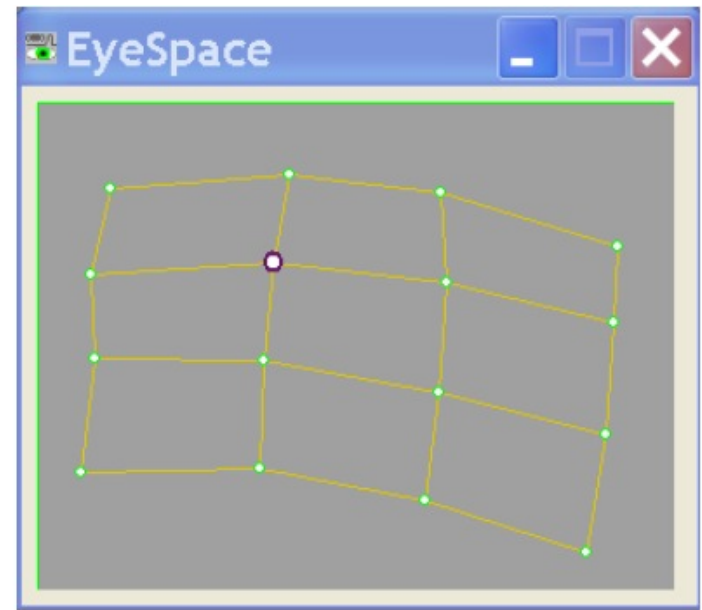

(b)

Fig. 2.6 Step-and-increment calibration mode. (a)Video frame recorded by the scene camera in "GazeSpace" window. The point being calibrated appears in blue. (b) "EyeSpace" window image showing a successful calibration. The point being calibrated appears white.

Fig. 2.6a shows how the "GazeSpace" window appeared in the computer monitor while the calibration was being performed. Fig. 2.6b is an example of the "EyeSpace" window showing 16 rectilinear well-spaced points indicating a successful calibration. The accuracy of the calibration was tested by asking the subject to gaze at the center of the projection screen and then follow the laser spot as it was moved around the screen and noting whether the gaze position was coincident with it. In the event that the subject moved his or her head during the calibration, or the tracking system moved with 
respect to the subject's head during the measurement, the eye-tracking software permitted "slip correction". This feature consisted of relocating the calibration data set so that the gaze position as recorded by the computer once more corresponded to the actual position of the gaze.

\subsection{Subjects}

Subjects consisted of volunteers recruited primarily from among the students and staff at Florida International University. The subjects fell into two categories: naïve and informed. Five informed subjects were familiarized with the study including its goals and hypotheses, and were employed in initial feasibility tests. The rest of the subjects $(n=58)$ were told only that the study was to determine the distribution of light on the retina. In particular they were never prompted to gaze at any specific point in their visual field during the tests. The ages of the naïve subjects ranged from 8 years old to 61 years old ( $29 \pm 15$ years) and were subdivided into three groups: Group A consisted of 24 subjects under 21 years old ( $17 \pm 3$ years). Sixteen subjects between 21 years old and 35 years old ( 25 \pm 3 years) formed Group B. Group C included 18 subjects above 35 years old ( $49 \pm 9$ years). All subjects signed informed consent forms approved by the University's Institutional Review Board and were apprised both verbally and in writing of the nature and potential risks of the experiments. They were provided with a copy of an IRB-approved Informed Consent Form. The form included a section for parental permission in the case of subjects under 18 years of age. Additionally, subjects were informed of their right to discontinue participation in the study at any time. Each subject was identified by his/her initials and five more characters indicating the age group (A, B or C) the Phase number (P1, P2, P3, P4, and P5) and two numbers indicating the order in which the data were taken. For example, identifier P1A05GS, corresponded to the data taken in Phase 1 from the fifth subject who had initials GS, and age $<21$ (A). Data and records obtained from individual subjects were stored on a secured PC, and printed copies were stored in a locked filing cabinet in the lab. All research procedures conformed to the tenets of the Declaration of Helsinki. 


\subsection{Validation of the Method}

Experiments conducted with the informed subjects consisted of approximately 10 minute sessions during which the subjects were seated in front of the projection screen. The uniformity of the image projected was verified with a Minolta Spotmeter. A PowerPoint presentation, consisting of a sequence of 78 photographic images (See Appendix 1), was projected on the screen with each image being visible for 3 seconds followed by a 1 second period during which the screen was blank. These photographic images were taken with a Canon EOS 400D (EF-S18-55mm, f/3.5-5.6 II) digital camera from several areas around campus. Each image was loaded into MATLAB® (Guide 1998), and corrected for vignetting, which is a spatial decrease in illumination from the center of the image introduced by the camera's lens ( $\mathrm{Yu}$ 2004). The correction was performed by subtracting the flat-field shown in Fig. 2.7 from each original image. The flat field was obtained by photographing a blank white screen under sunlight illumination. Fig. 2.8 shows an example of an original image and the corresponding image after correction.

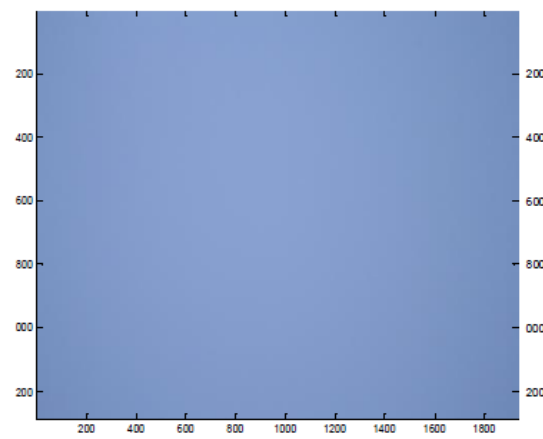

(a)

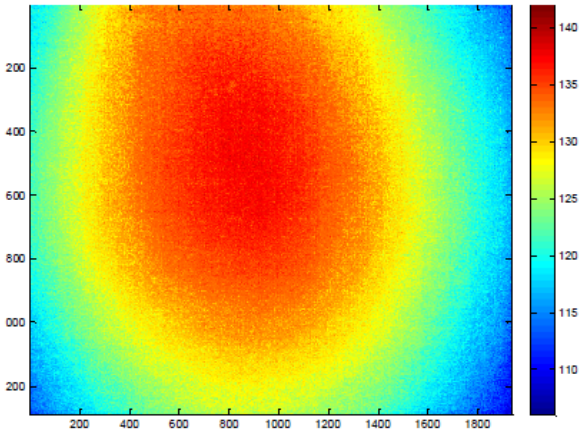

(b)

Fig. 2.7. "Flat-white" image used for vignetting correction. (a) actual image (b) intensity map.

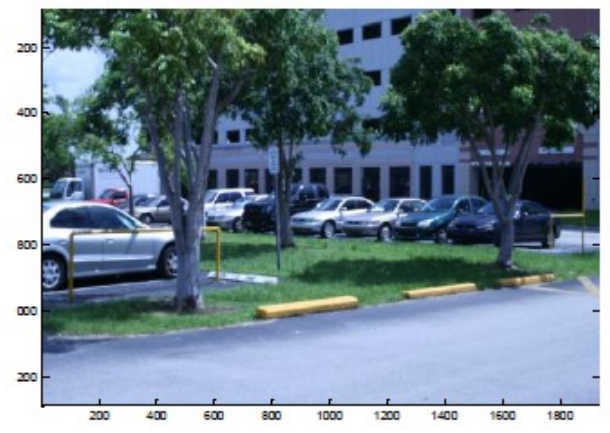

(a)

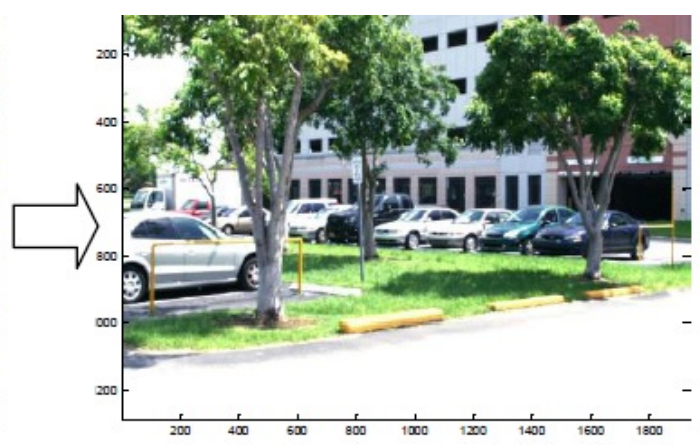

(b)

Fig. 2.8. Example of vignetting correction (a) uncorrected image (b) corrected image. 
Subjects were asked to direct their gaze immediately at the brightest part of each slide when it appeared. In a second series of sessions, the subjects were asked to fixate on the darkest part of each photograph. In an additional test, two of the informed subjects were asked to fixate on bright and dark objects, respectively, while walking freely around the lab.

\subsection{Experiments with Naïve Subjects}

The experiments performed with naïve subjects consisted of 5 different sessions or phases, each session being less restricted than the previous one. In phases 1, 2 and 3 the subject's head was fixed but there was free viewing conditions in phases 4, and 5. In Phase 1 (Static Image) the subjects spent $\sim 3$ minutes viewing the gray-scale image shown in Fig. 2.3a. In Phase 2 (PowerPoint Presentation) the subjects spent 3 to 6 seconds viewing each image in the PowerPoint presentation described in the previous section. In Phase 3 (Movie) the subject watched a video clip for $\sim 8$ minutes. The video clip was composed of short segments taken from several areas around the campus and recorded with a Sony HDR-XR200 high definition video camera $(1920$ x 1080). For all participants, this part of the study was performed immediately after Phase 2 was completed. In Phase 4 (Computer) the subjects were asked to sit in front of a 15 inch laptop computer and perform random tasks such a reading their emails or surfing the internet. The recording times in Phase 4 were 5, 10 and 15 minutes. Phase 5 was subdivided in two sessions. In one session (Lab) the subject was asked to walk freely around the lab and surrounding hallways for a period of 5 to 7 minutes. The last session (Outside) only included young subjects (age $<21$ ) and consisted of 3 to 7 minute walks around the campus. The eye-tracker was connected to the computer by a long cable, and the computer itself, which was powered by a backup battery, was placed on a small cart which was pushed behind the subjects as they proceeded with their walk. All subjects walked around approximately the same areas of the FIU campus. In this experiment, the dark pupil effect required for eye tracking was strongly influenced by the intensity of ambient infrared light in the environment relative to the intensity of the IRED, producing unwanted corneal reflections on the surface of the eye. This issued was minimized 
by incorporating a light-weight, Makrolon ${ }^{\circledR}$ infrared blocking face shield that was used as a sunblocking brim.

\subsection{Data Analysis.}

The data recorded by the eye tracker consisted of MPEG files containing the pixel values of each 360 by 240 frame in the video recorded by the scene camera, and the corresponding Excel files containing the $x$ and $y$ coordinates of the gaze positions (normalized with respect to the horizontal and vertical axes of the movie frame as captured by the scene camera). Also recorded were the pupil dimensions. A MATLAB ${ }^{\circledR}$ program was written to process the data. See Appendix 1.

The gray-scale image used in Phase 1, and each video frame obtained in Phases 2, 3, 4 and 5 were assumed to result in an undistorted image on the retina positioned so that the pixel corresponding to the recorded gaze position was centered on the fovea. See Fig. 2.9 for a hypothetical example.
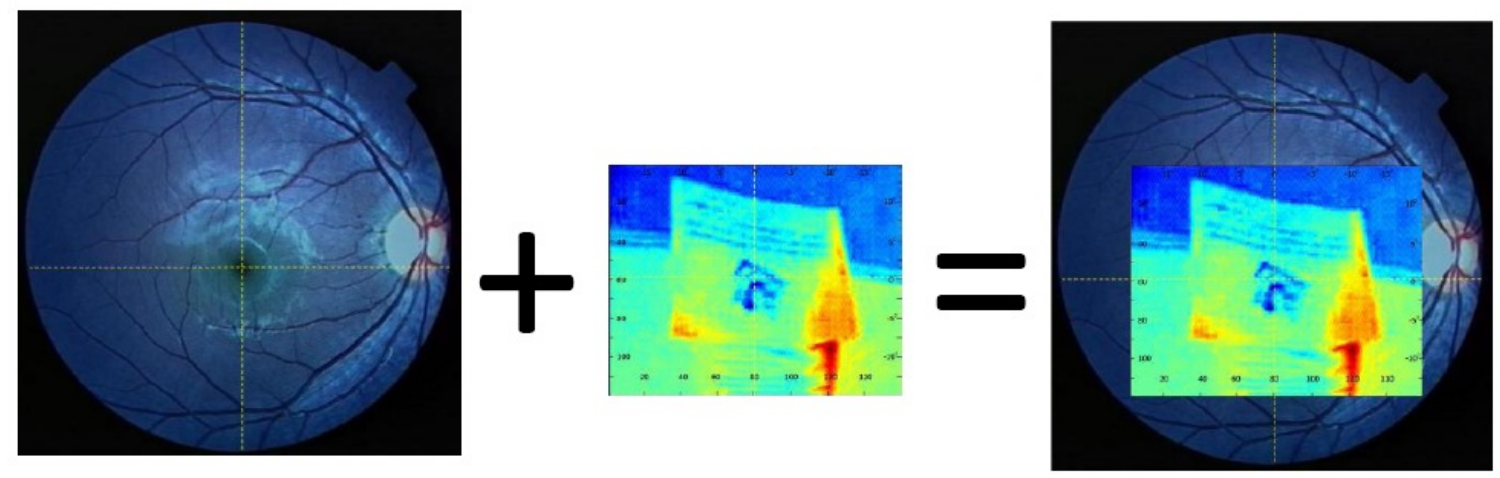

Fig 2.9. Hypothetical example showing a video frame projected on the retina. Dimensions are approximate.

The relative (rather than absolute) illuminance on the retina was assumed to be the same as the relative illuminance recorded in the video frame. This assumption was based on wide-angle models of the eye that show that, for Ganzfeld illumination, the retinal illuminance, is uniform from $0^{\circ}$ to $\sim 25^{\circ}$ of eccentricity. (Kooijman 1983; Pflibsen, Pomerantzeff et al. 1988)This study did not take into account the absorption of short-wavelength light by the lens (Van Norren and Vos 1974) which reduces the amount of blue light that reaches the retina. 
Fig. 2.10a shows four actual frames (Frames 0001, 1000, 2000 and 2500) as recorded by the scene camera. Each frame contains one gaze position indicated by the green dot. If the subject's gaze position fell outside the boundary of a central 160 by 120 pixel rectangular area of a particular frame, the frame was rejected by the program. This step was necessary because part of the retina would be
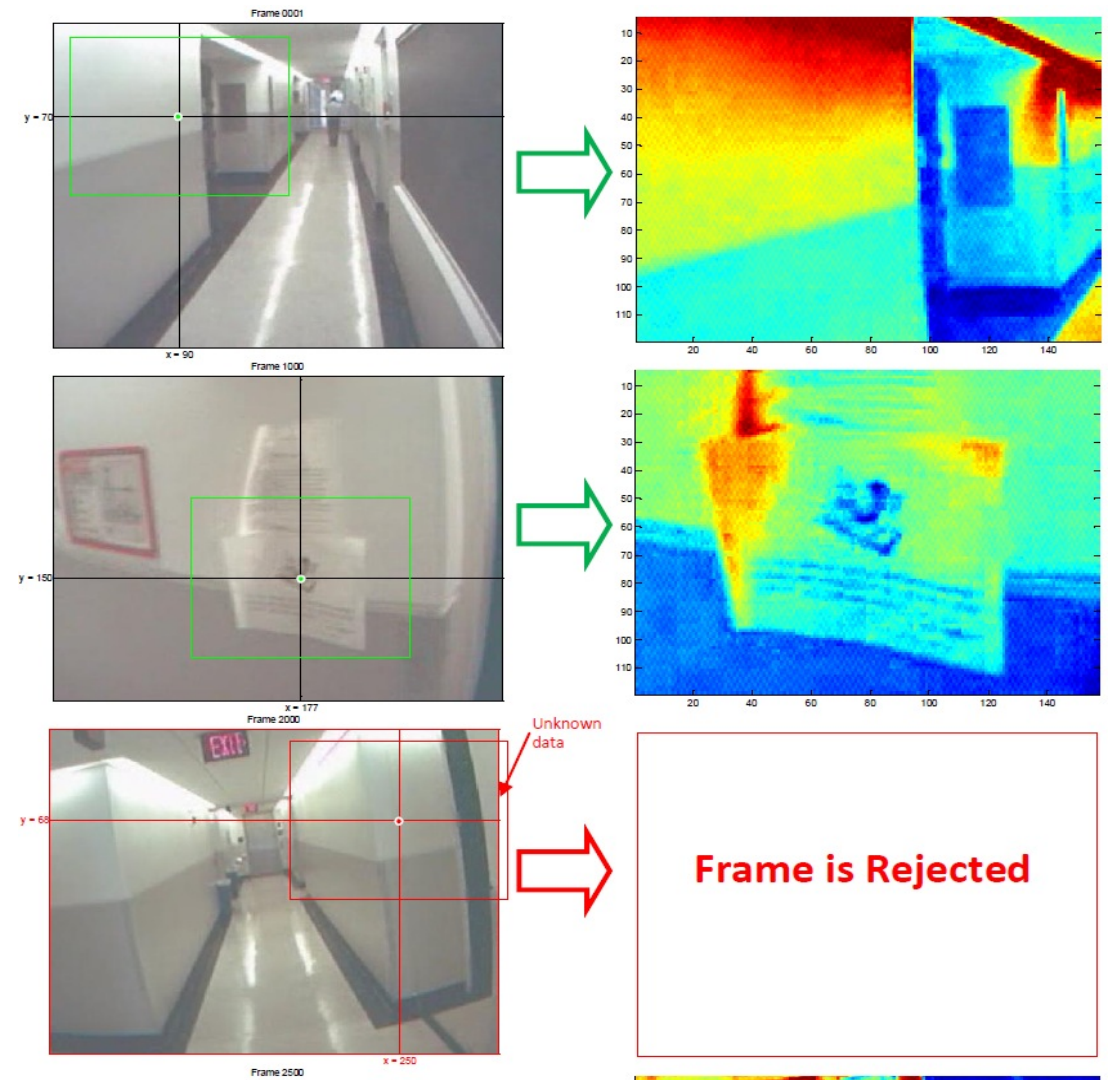

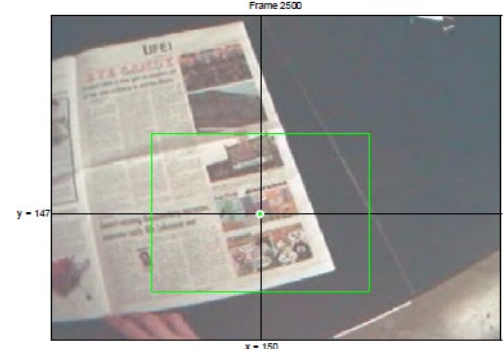

(a)

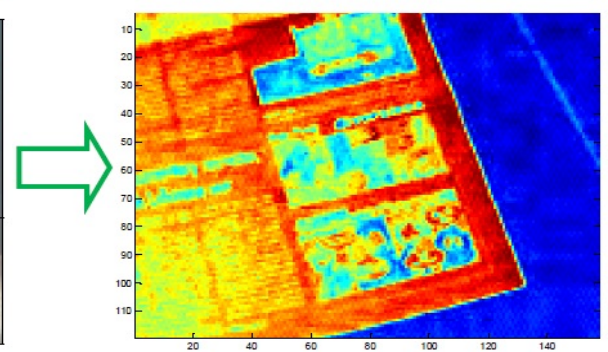

(b)

Fig. 2.10. Data recording and processing. (a) Four actual frames as recorded by the scene camera. Gaze position is indicated by the green circle. (b) Intensity maps produced by the region bounded by the green rectangle. Frame 2000 is rejected.

receiving light not accounted for by the pixel values contained within the video, but rather from the surroundings. Consequently, only those portions of each frame in the coordinate range -80 to +80 pixels in the $\mathrm{x}$ direction and -60 to +60 pixels in the $\mathrm{y}$ direction were included. These regions are 
represented by the green rectangles in Fig. 2.10a. Figure $2.10 \mathrm{~b}$ shows the intensity maps obtained from the regions bounded by the green rectangles shown in Fig 2.10a. Frame 2000 was rejected because the gaze position fell outside the central rectangle therefore containing information not accounted for. The ratio of the total number of frames contained in the video to the number of "useful" frames was used as another criterion to test the calibration of the system. If, for a particular video, the number of useful frames was less than $25 \%$ of the total number of frames, the entire video was visually inspected. If during the inspection the video revealed any sign of miscalibration (for example, the scene camera was not pointing forward for prolonged periods of time) then the video was rejected and the experiment was repeated.

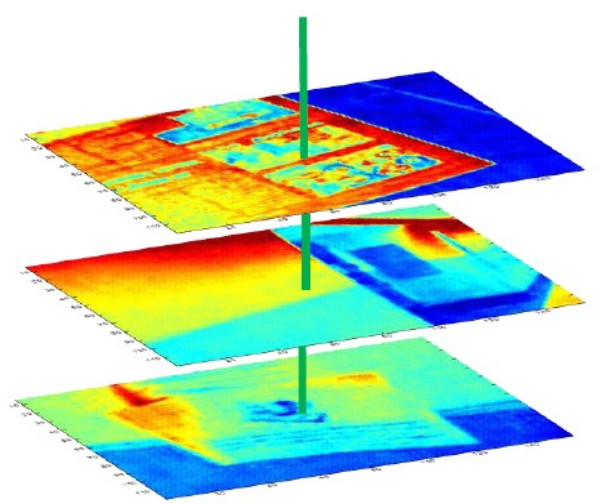

Fig. 2.11. Intensity maps from Fig. 2.10b. The intersection of the green line with each map indicates the subject's gaze position.

To create the retinal intensity map, the program converted each video frame from RGB to grayscale (0-255) and multiplied every pixel value by a factor representing the area of the pupil. In order to obtain the cumulative retinal illuminance, each map was aligned (See Fig. 2.11) based on the gaze position, and for all the useful frames, pixel values sharing the same pair of coordinates were added. The result of these operations was a 160 by 120 map, centered at the fovea, with each pixel value now representing the cumulative illuminance at the corresponding point on the retina. The pixel values in this map were normalized to a maximum value of 1.0 . Finally the dimensions, 160 by 120 , were converted to degrees of visual angle, according to the angular aperture of the scene camera lens. The angular aperture of the scene camera used in Phase 4 was $90^{\circ}$. The angular aperture of the scene 
camera used in all other phases was $67^{0}$. To verify the angular dimensions of the visual field, actual videos of the projection screen (with a ruler across its center) were recorded for different viewing distances. The angular dimensions on the video frames were compared to the angular dimensions on the screen. All the data produced by the MATLAB ${ }^{\circledR}$ program were stored in Excel files for further analysis. The MATLAB program codes are shown in Appendices 2, 3 and 4. Statistical analyses were performed using $\operatorname{IBM}{ }^{\circledR} \operatorname{SPSS}{ }^{\circledR}$ Statistics Version 21. For phases 1 and 4 , the mean retinal illuminance was calculated for a $1^{0}$ disk around the fovea and within a $1^{0}$ wide annulus at $14^{0}$ of radial eccentricity, and the difference in retinal illuminance between these two retinal locations was analyzed by a one-way analysis of variance (ANOVA) followed by Turkey's test. 


\section{RESULTS}

\subsection{Validation of the Method}

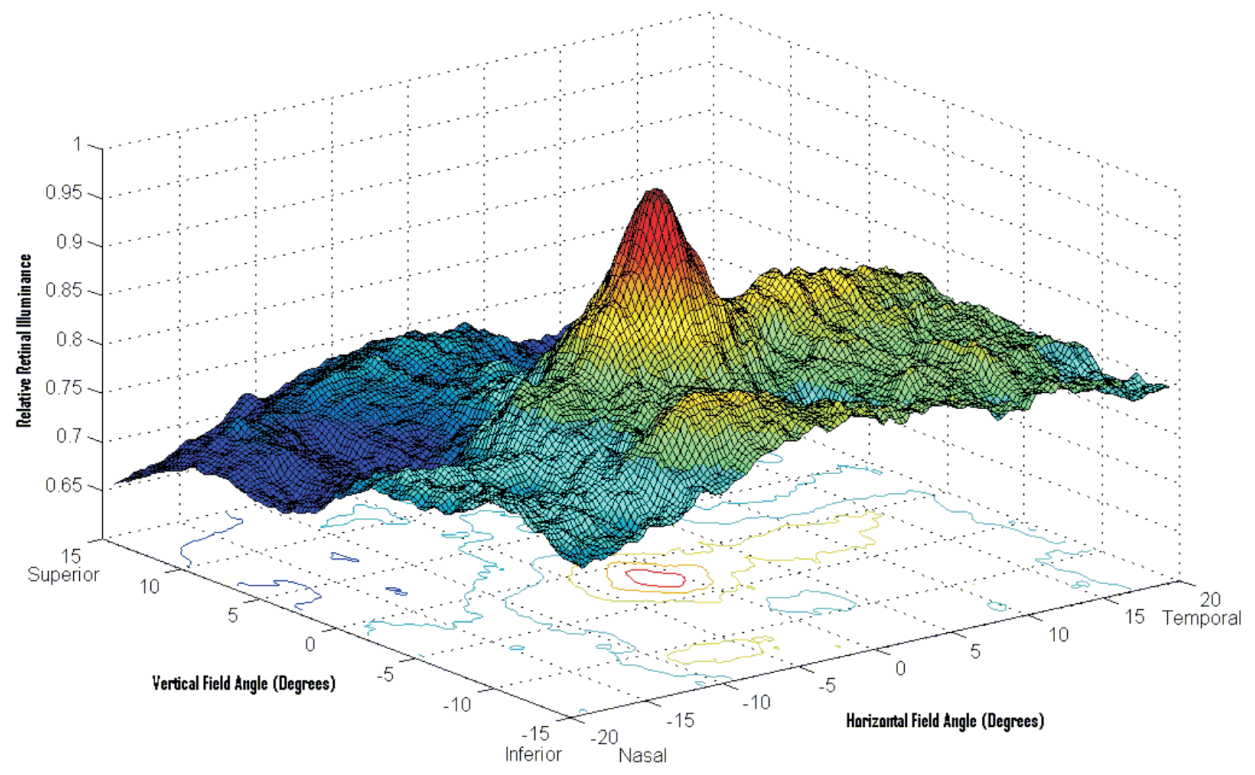

Fig. 3.1. Mesh plot showing the cumulative light distribution (relative) on the retina for an informed subject instructed to look at the brightest objects in the field of view while walking freely around the lab.

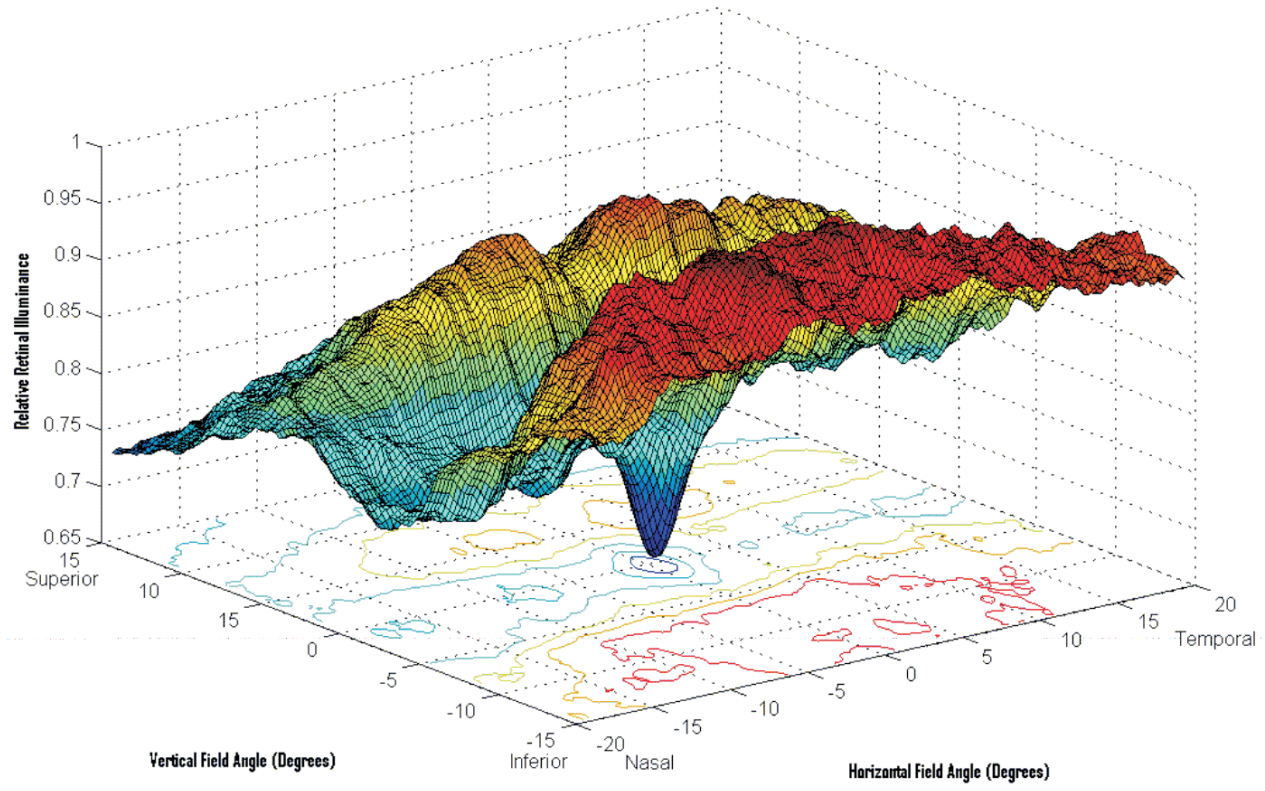

Fig. 3.2. Mesh plot showing the cumulative light distribution (relative) on the retina for an informed subject instructed to look at the darkest objects in the field of view while walking freely around the lab. 
Figs. 3.1 and 3.2 are three-dimensional mesh plots that show the results of the experiment in which an informed subject was asked to fixate on bright and dark objects respectively while walking freely around the lab. In both graphs the vertical axis represents the cumulative light distribution (relative) on the retina while the horizontal axes represent displacement from the foveal center $(0,0)$ in the temporal-nasal and superior-inferior directions. The central peak located at the fovea in Fig. 3.1 is a clear indication that the subject was fixating on the brightest objects. Similarly, the dip in retinal illumination at the foveal location in Fig. 3.2 shows that the subject was fixating on the darkest objects in the field of view. The illumination on the inferior portion of the retina for both graphs is slightly higher than the illumination of the superior portion of the retina because of ceiling lighting. (Recall that the image is inverted.) No significant differences were observed on the horizontal temporal-nasal line crossing the fovea. These results consistently verified that the eye-tracker system, the calibration, the procedures, and the computational processing of the data were functioning as intended. Similar results were obtained for the other four informed subjects.

\subsection{Experiments with naïve subjects.}

Results for each naïve subject included: a) maps showing the cumulative light distribution (relative) on the retina with horizontal and vertical axes representing displacement from the foveal center $(0,0)$ in the nasal-temporal and superior-inferior directions respectively; b) plots of the mean retinal illuminance calculated for 28 radial $\left( \pm 0.25^{\circ}\right)$ eccentricities from the fovea; and c) horizontal line-scans through the fovea, which were obtained to analyze the illumination patterns without taking into consideration the effects of high illumination in the inferior region of the retina (e.g. due to ceiling lighting or skylight). For $b$ ), the mean retinal luminance between radial distances $r$ and $r+\Delta r$ was calculated as the average pixel value in this range.

For the 30 subjects viewing a gray-scale image projected on the screen (Phase 1), 16 subjects had the highest illuminance at the foveal center. See 3.3a. For 11 subjects, the illumination maps often revealed some areas of increased illuminance in the center of the retina, but the illuminance maximum was found in the nasal-superior quadrant. See Fig. 3.3b. For the remaining 3 subjects, the 
maximum retinal illuminance was located away from the central retina in the nasal direction. See Fig.

3.3c. For the overall average retinal illumination map, the maximum was displaced about $1^{0}$ horizontally and $5^{0}$ vertically in the temporal-superior direction. See Fig. 3.4.

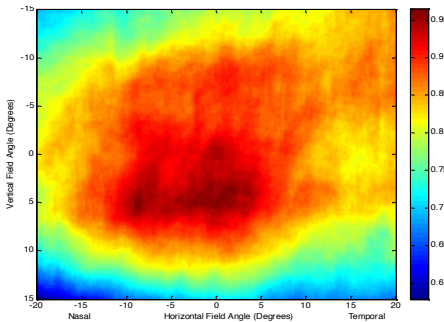

(a)

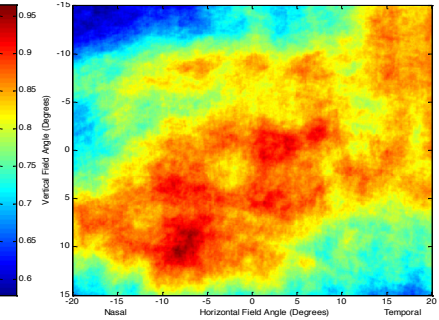

(b)

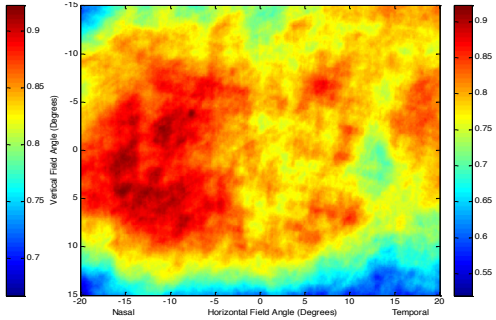

(c)

Fig. 3.3. Intensity maps showing the average cumulative light distribution (relative) on the retina for naïve subjects viewing the gray-scale shown in Fig.3.3a. (a) 16 subjects (b) 11 subjects (c) 3 subjects.

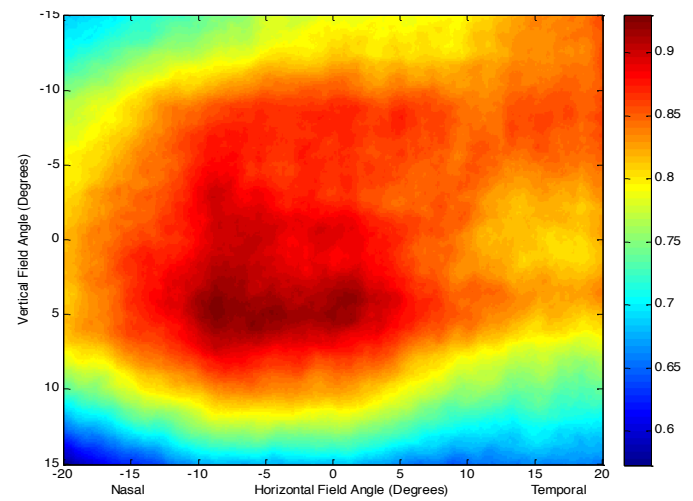

Fig. 3.4. Map showing the average cumulative light distribution (relative) on the retina for all 30 naïve subjects viewing a gray-scale image.

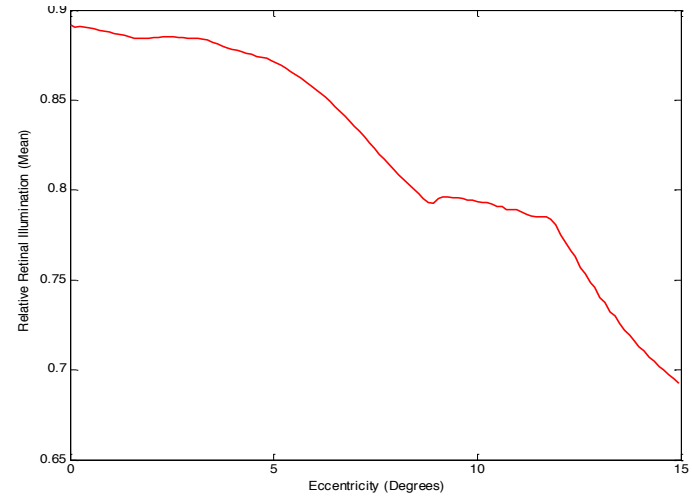

(a)

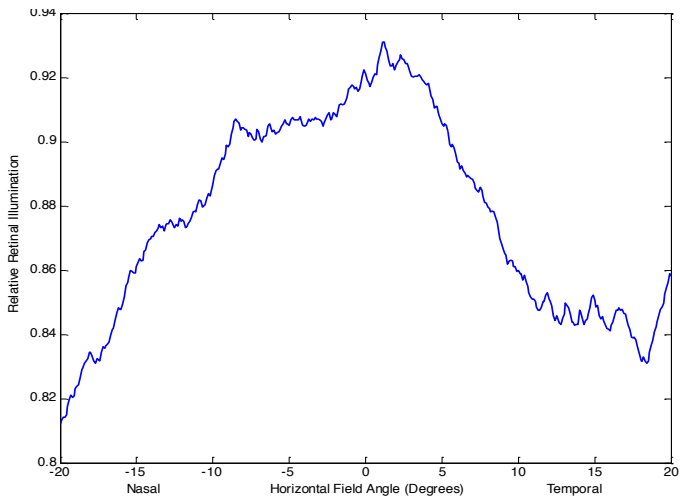

(b)

Fig. 3.5. (a) Mean value of retinal illuminance versus radial eccentricity $\left( \pm 0.25^{\circ}\right)$ derived from Fig. 3.4. (b) Horizontal line-scan through the foveal region in Fig. 3.4. 
Mean values of retinal illuminance versus radial eccentricity $\left( \pm 0.25^{\circ}\right)$ derived from Fig. 3.4 showed a $\sim 20 \%$ decrease in retinal illuminance with eccentricity from the fovea. See Fig. 3.5a. The horizontal line-scan through the foveal region in Fig. 3.4 showed a $\sim 10 \%$ decrease of retinal illuminance in both nasal and temporal directions. Both the mean value versus eccentricity (Fig. 3.5a) as well as the horizontal line profile (Fig. 3.5b) peaked at the foveal center.

For the 20 subjects viewing a PowerPoint presentation of photographic images (Phase 2), 11 subjects had the highest illuminance in the nasal direction (see 3.6a) while 9 subjects showed a small increase in illuminance around the fovea, but in general the highest illumination was found $\sim 15^{\circ}$ in the superior direction. See Fig. 3.6b. When averaged for all subjects, there was a small region of increased illuminance at the fovea but the maximum illuminance was elsewhere. See Fig. 3.7.

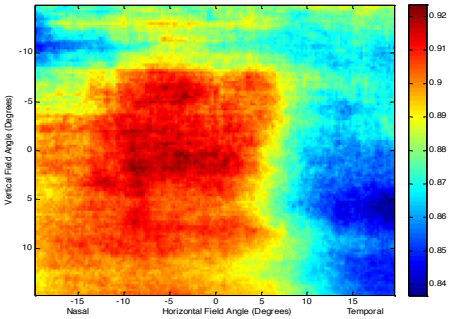

(a)

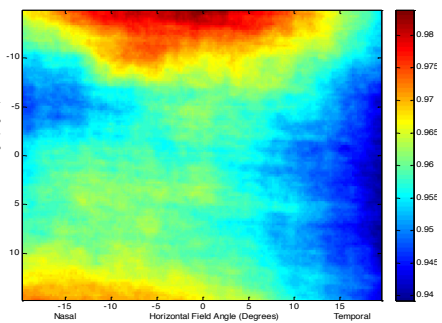

(b)

Fig. 3.6. Intensity maps showing the average cumulative light distribution (relative) on the retina for naïve subjects viewing a PowerPoint presentation of photographic images. (a) 11 subjects (b) 8 subjects.

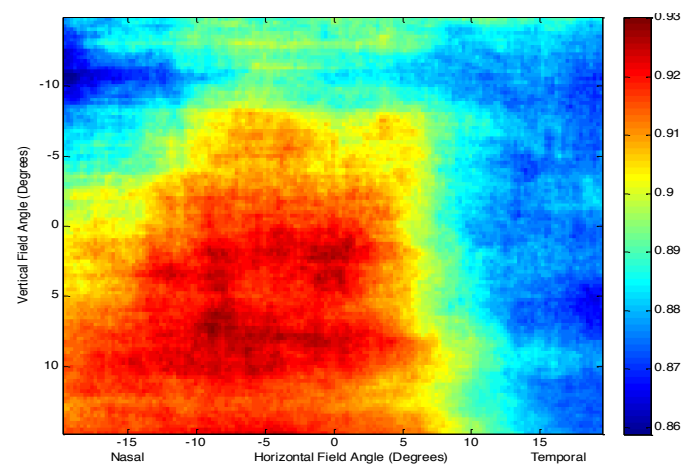

Fig. 3.7. Map showing the average cumulative light distribution (relative) on the retina for naïve subjects viewing a PowerPoint presentation of photographic images.

The mean value of retinal illuminance vs. eccentricity derived from Fig. 3.7 shows a small, relatively uniform decrease in retinal illumination with eccentricity. See Fig. 3.8a. The horizontal line- 
scan through the foveal region in shown in Fig. $3.8 \mathrm{~b}$ and indicates a very small, decrease $(\sim 2 \%)$ in the nasal direction and a small decrease $(\sim 5 \%)$ in the temporal direction from the fovea.

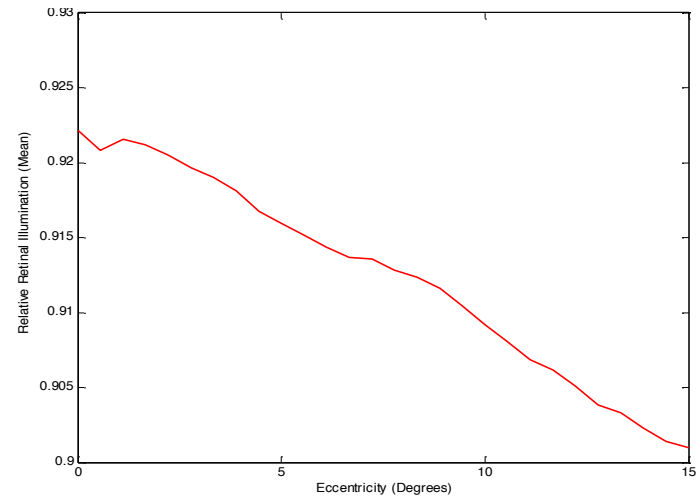

(a)

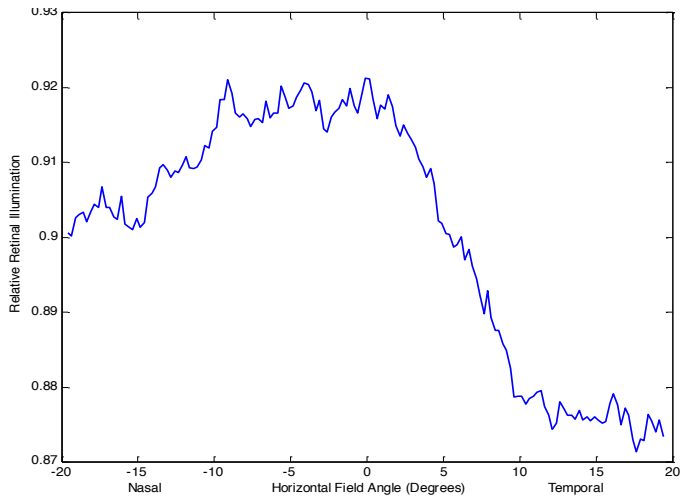

(b)

Fig. 3.8. (a) Mean values versus radial eccentricity $\left( \pm 0.25^{\circ}\right)$ derived from Fig. 3. (b) Horizontal linescan through the foveal region in Fig. 3.7.

For the 15 naïve subjects viewing a video presentation (Phase 3), 4 showed a maximum in the central foveal region (see Fig. 3.9a). For 8 subjects, there was a region of higher illuminance at the fovea but the maximum was found at $\sim 14^{\circ}$ from the fovea in the inferior direction. See Fig. 3.9b. The average illuminance map for the remaining 3 subjects indicated a broad region of higher illuminance in the temporal-inferior quadrant. See Fig. 3.9c.

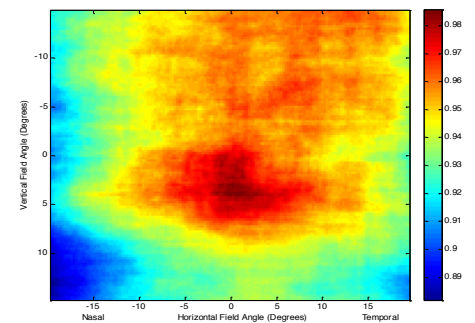

(a)

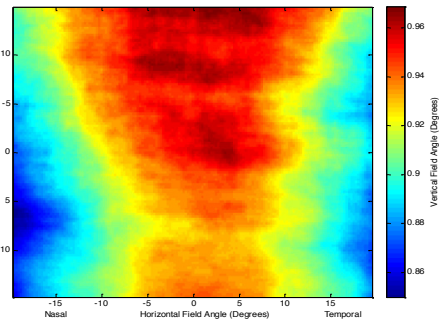

(b)

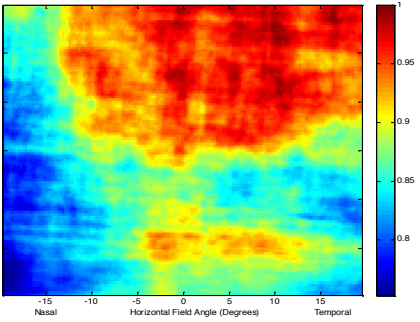

(c)

Fig. 3.9. Intensity maps showing the average cumulative light distribution (relative) on the retina for naïve subjects viewing a video presentation. (a) 4 subjects (b) 8 subjects (c) 3 subjects. 


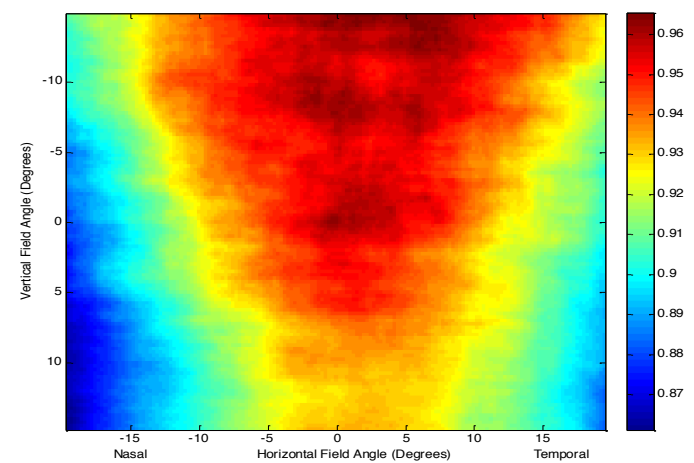

Fig. 3.10. Map showing the average cumulative light distribution (relative) on the retina for naïve subjects viewing a video presentation.

The overall average map for all subjects indicated an increased illuminance in the center of the retina (See Fig. 3.10), but the average peak was not necessarily centered at the fovea but rather towards the inferior direction. The mean value of retinal illumination indicated a very small $(\sim 4 \%)$ decrease with eccentricity from the fovea. See Fig. 3.11a. The horizontal line-scan showed a small $(\sim 8 \%)$ increase in illuminance at the fovea. See Fig. 3.11b.

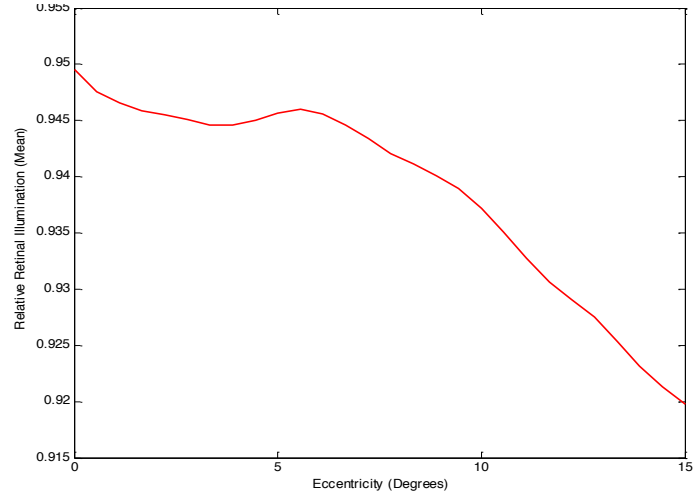

(a)

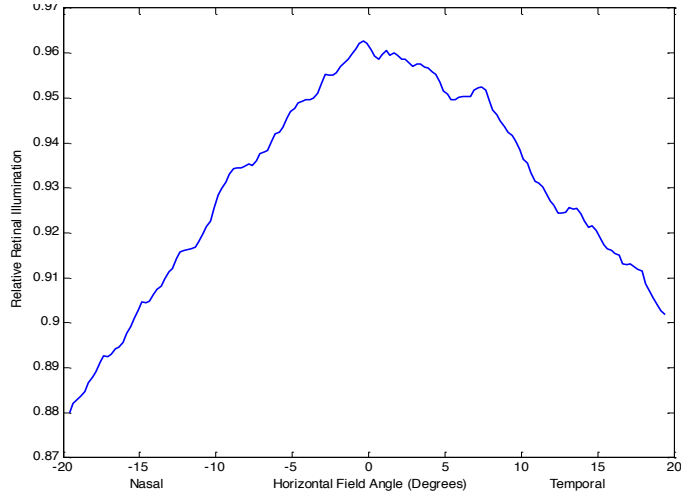

(b)

Fig. 3.11. (a) Mean values versus radial eccentricity $\left( \pm 0.25^{\circ}\right)$ derived from Fig. 3.10. (b) Horizontal line-scan through the foveal region in Fig. 3.10.

The most consistent results were obtained in Phase 4 where the subjects viewed a computer monitor for periods of 5, 10 and 15 minutes. In every case, there was a pronounced maximum in retinal illuminance at the foveal center. See Fig. 3.12. Both the mean value (Fig. 3.13a) and the horizontal line-scan (Fig. 3.13b) also showed a pronounced maximum at the foveal center with a smooth decrease towards the periphery. 


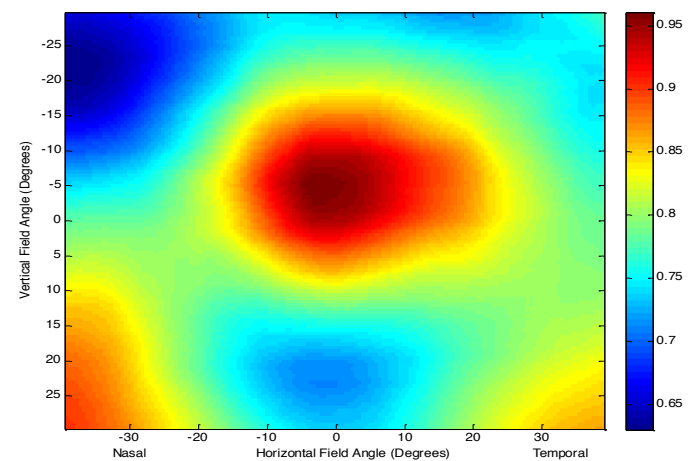

Fig. 3.12. Map showing the average cumulative light distribution (relative) on the retina for naïve subjects viewing a video computer monitor.

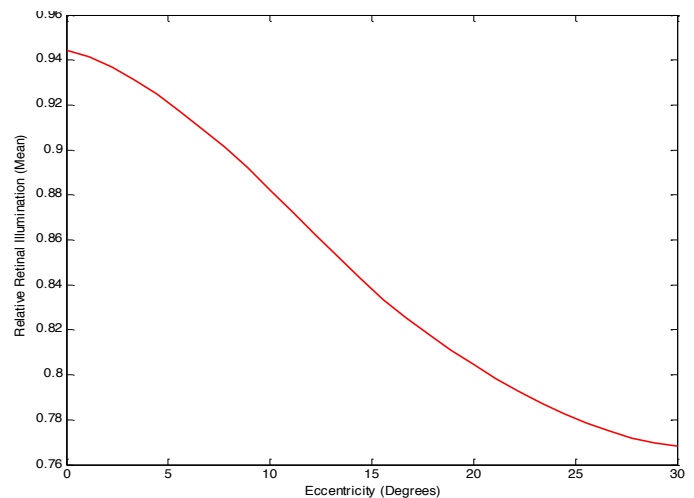

(a)

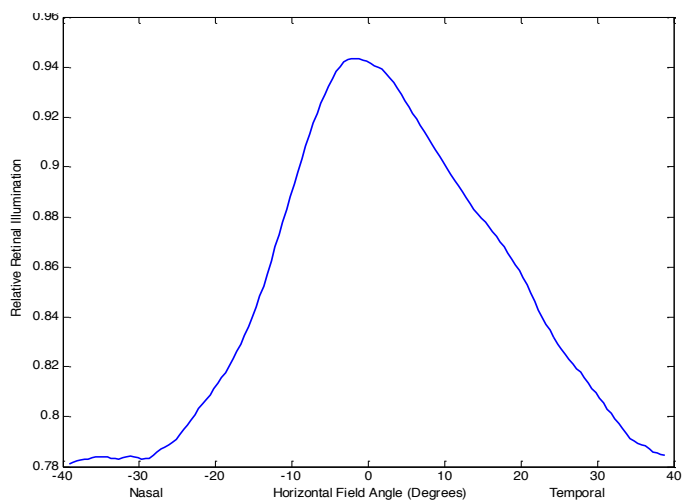

(b)

Fig. 3.13. (a) Mean values versus radial eccentricity $\left( \pm 0.5^{\circ}\right)$ derived from Fig. 3.12. (b) Horizontal line-scan through the foveal region in Fig. 3.12 showing clear maximum at the foveal center.

Table 3.1 shows the analysis of the mean values of the difference in retinal illuminance within $1^{0}$ around the fovea and at $15^{\circ}$ of radial eccentricity for the three measuring times. The results of the one-way ANOVA and the post hoc Turkey's test revealed no statistically significant difference among the three measuring times. See Tables 3.2 and 3.3. 


\begin{tabular}{|c|c|c|c|c|c|c|c|c|}
\hline \multirow[t]{2}{*}{$\begin{array}{l}\text { Measuring } \\
\text { Time }\end{array}$} & \multirow[t]{2}{*}{$\mathrm{N}$} & \multirow[t]{2}{*}{ Mean } & \multirow[t]{2}{*}{$\begin{array}{c}\text { Std. } \\
\text { Deviation }\end{array}$} & \multirow[t]{2}{*}{$\begin{array}{l}\text { Std. } \\
\text { Error }\end{array}$} & \multicolumn{2}{|c|}{$\begin{array}{l}95 \% \text { Confidence } \\
\text { Interval for Mean }\end{array}$} & \multirow[t]{2}{*}{ Minimum } & \multirow[t]{2}{*}{ Maximum } \\
\hline & & & & & $\begin{array}{l}\text { Lower } \\
\text { Bound }\end{array}$ & $\begin{array}{l}\text { Upper } \\
\text { Bound }\end{array}$ & & \\
\hline 5 minutes & 7 & .1978 & .06271 & .02370 & .1398 & .2558 & .10 & .29 \\
\hline 10 minutes & 9 & .1917 & .05140 & .01713 & .1522 & .2312 & .12 & .30 \\
\hline 15 minutes & 9 & .1746 & .05327 & .01776 & .1336 & .2155 & .06 & .23 \\
\hline Total & 25 & .1872 & .05394 & .01079 & .1650 & .2095 & .06 & .30 \\
\hline
\end{tabular}

Table 3.1. Analysis of the mean values of the difference in retinal illuminance within $1^{\circ}$ around the fovea and at $15^{\circ}$ of radial eccentricity for three viewing times.

\begin{tabular}{|l|r|r|r|r|r|}
\hline & Sum of Squares & df & Mean Square & F & Sig. \\
\hline Between Groups & .002 & 2 & .001 & .392 & .680 \\
Within Groups & .067 & 22 & .003 & & \\
Total & .070 & 24 & & & \\
\hline
\end{tabular}

Table 3.2. One-way ANOVA results for three viewing times.

\begin{tabular}{|c|c|c|c|c|c|c|}
\hline \multirow{2}{*}{$\begin{array}{l}\text { (I) Recording } \\
\text { Time }\end{array}$} & \multirow{2}{*}{$\begin{array}{l}\text { (J) Recording } \\
\text { Time }\end{array}$} & \multirow{2}{*}{$\begin{array}{c}\text { Mean } \\
\text { Difference } \\
(\mathrm{I}-\mathrm{J}) \\
\end{array}$} & \multirow{2}{*}{$\begin{array}{l}\text { Std. } \\
\text { Error }\end{array}$} & \multirow[t]{2}{*}{ Sig. } & \multicolumn{2}{|c|}{ 95\% Confidence Interval } \\
\hline & & & & & $\begin{array}{l}\text { Lower } \\
\text { Bound }\end{array}$ & $\begin{array}{l}\text { Upper } \\
\text { Bound }\end{array}$ \\
\hline \multirow{2}{*}{5 minutes } & 10 minutes & .00606 & .02790 & .974 & -.0640 & .0761 \\
\hline & 15 minutes & .02322 & .02790 & .687 & -.0469 & .0933 \\
\hline \multirow{2}{*}{10 minutes } & 5 minutes & -.00606 & .02790 & .974 & -.0761 & .0640 \\
\hline & 15 minutes & .01716 & .02610 & .790 & -.0484 & .0827 \\
\hline \multirow{2}{*}{15 minutes } & 5 minutes & -.02322 & .02790 & .687 & -.0933 & .0469 \\
\hline & 10 minutes & -.01716 & .02610 & .790 & -.0827 & .0484 \\
\hline
\end{tabular}

Table 3.3. Multiple comparison post hoc Turkey's test for three viewing times.

For naïve subjects for whom data were recorded under free viewing conditions as they walked around the lab and hallways (Phase 5), 6 had the highest illuminance at the foveal center. See Fig. 3.14a. For 10 subjects, the illumination maps revealed broad areas of increased illuminance in the center of the retina, but the illuminance maximum was displaced from the center. See Fig. 3.14b. For 
8 subjects, the maximum retinal illuminance was located at the inferior region of the retina. See Fig. $3.14 \mathrm{c}$.

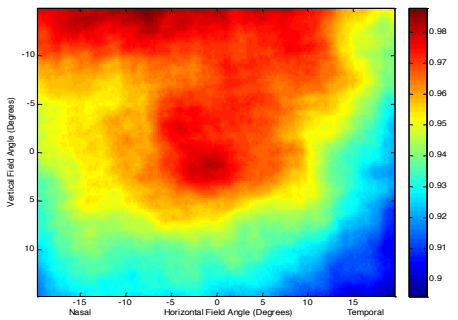

(a)

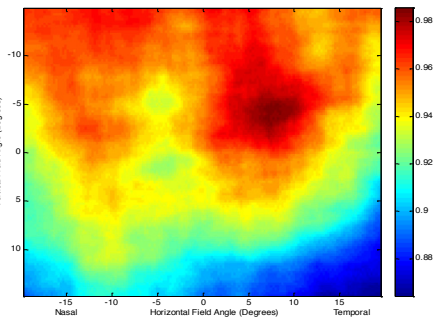

(b)

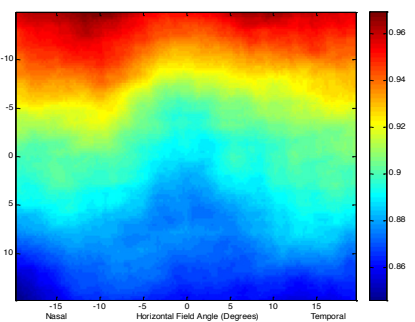

(c)

Fig. 3.14. Intensity maps showing the average cumulative light distribution (relative) on the retina for naïve subjects walking freely around the lab and surrounding hallways (a) 10 subjects (b) 8 subjects (c) 6 subjects.

The overall average map of all the subjects combined is shown in Fig. 3.15, and it exhibits a broad area of maximum retinal illumination towards the inferior direction. The mean value plot is shown in Fig. 3.16a. It shows a very small decrease ( 2\%) with eccentricity. The horizontal line-scan derived from Fig. 3.15 is almost flat but shows a $\sim 2 \%$ decrease with radial eccentricity. See Fig 4.16b.

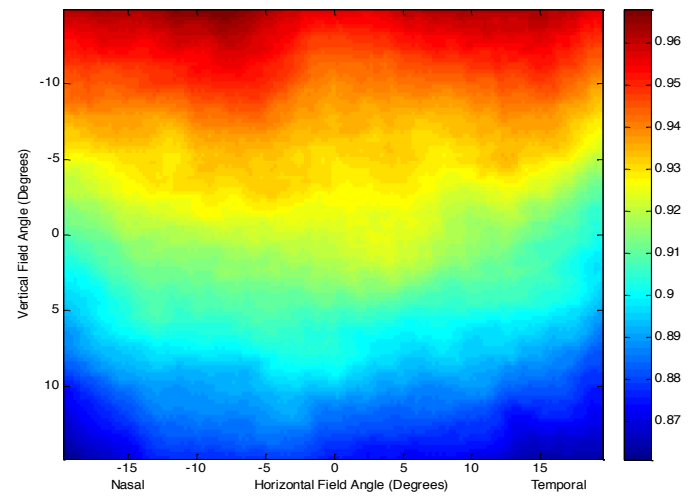

Fig. 3.15. Map showing the average cumulative light distribution (relative) on the retina for naïve subjects walking freely around the lab and surrounding hallways. 


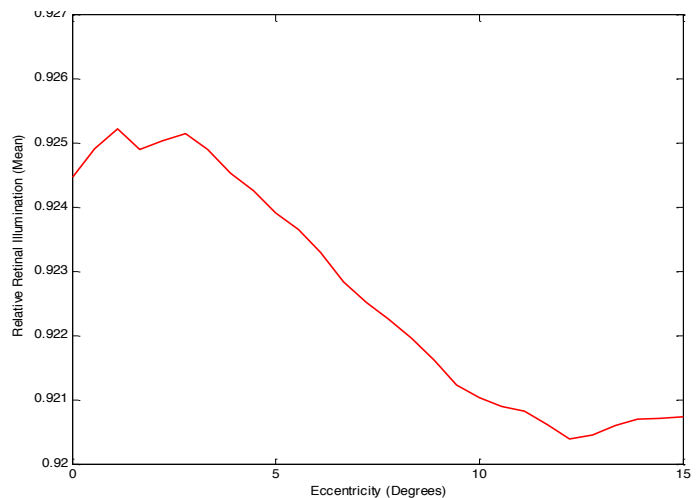

(a)

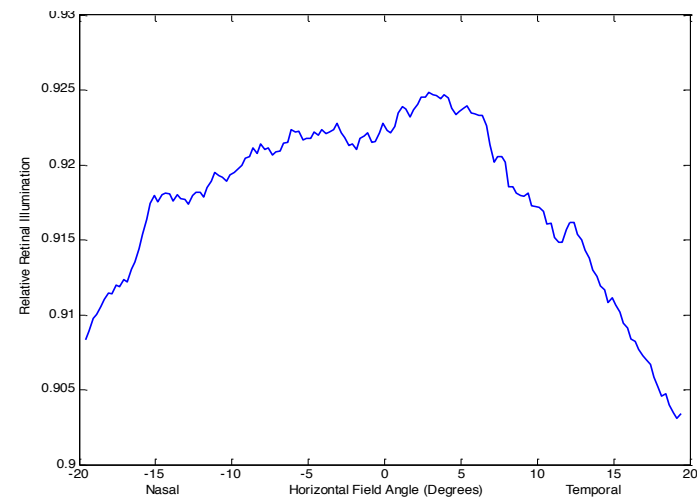

(b)

Fig. 3.16. (a) Mean values versus radial eccentricity $\left( \pm 0.25^{\circ}\right)$ derived from Fig. 3.15. (b) Horizontal line-scan through the foveal region in Fig. 3.15.

Finally, for the 10 young naïve subjects for whom data was recorded under free viewing conditions as they walked around campus (Phase 5), the results were very similar to the subjects that walked around the lab and hallways. Two subjects had the highest illuminance at the foveal center. The average map of these two subjects is shown in Fig. 3.17a. For 3 subjects, the illumination maps revealed some areas of increased illuminance in the center of the retina, but the illuminance maximum was displaced towards the inferior direction. See Fig. 3.17b. For the remaining 5 subjects, the maximum retinal illuminance was located away from the fovea towards the inferior direction. See Fig. 3.17c. The overall average map of all the subjects combined is shown in Fig. 3.18. Although it shows a small increase of retinal illumination around the fovea, the illuminance maximum was found in the superior direction. The mean value versus eccentricity is shown in Fig. 3.19a, and it shows a small decrease $(\sim 2 \%)$ with eccentricity. The horizontal line-scan shows a relatively constant retinal illuminance. See Fig. 3.19b.

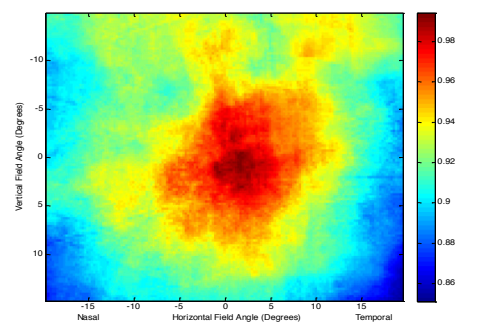

(a)

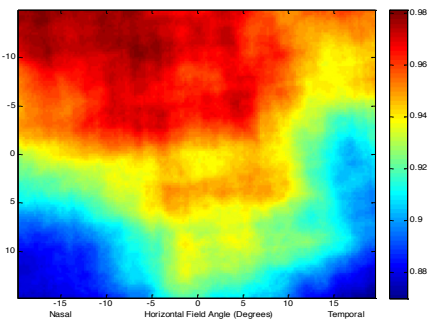

(b)

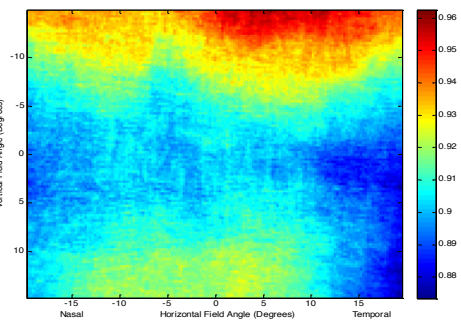

(c) 
Fig. 3.17. Intensity maps showing the average cumulative light distribution (relative) on the retina for naïve subjects walking freely around campus (a) 2 subjects (b) 3 subjects (c) 5 subjects.

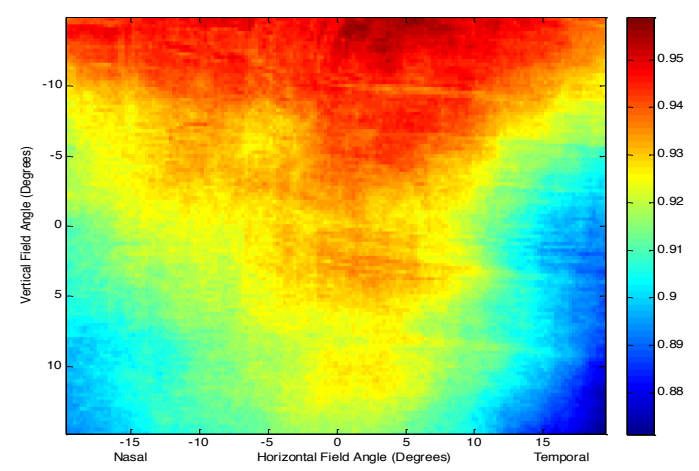

Fig. 3.18. Map showing the average cumulative light distribution (relative) on the retina for naïve subjects walking freely around campus.

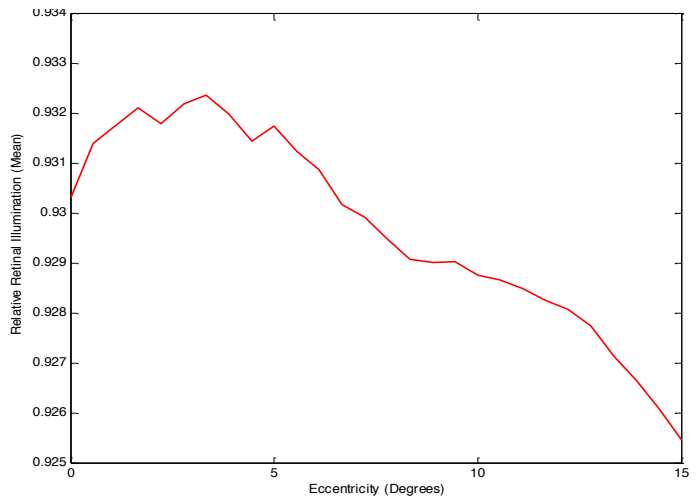

(a)

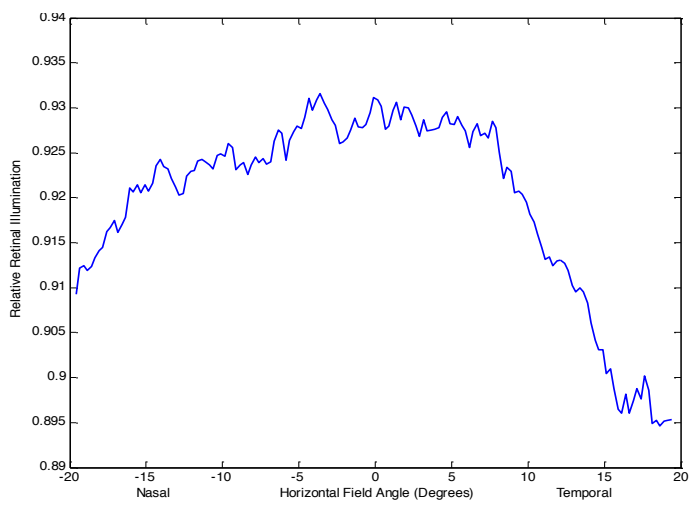

(b)

Fig. 3.19. (a) Mean values versus radial eccentricity $\left( \pm 0.25^{\circ}\right)$ derived from Fig 4.18 (b) horizontal line-scan through the foveal region in Fig. 3.18.

Fig. 3.20 shows the average illumination map for all subjects and all Phases combined. Although there is a small increase in illumination at the foveal center, the retinal illuminance maximum is located $\sim 13^{\circ}$ in the inferior direction. Fig. 3.21a represents the mean values versus eccentricity derived from Fig. 3.20. It shows a small ( 4\%) decrease in retinal illuminance with radial eccentricity. Fig. $3.21 \mathrm{~b}$ is the horizontal line-scan through the fovea in Fig. 3.20 showing a $\sim 4 \%$ increase at the foveal center with a reasonably uniform decrease in the temporal and nasal directions. 


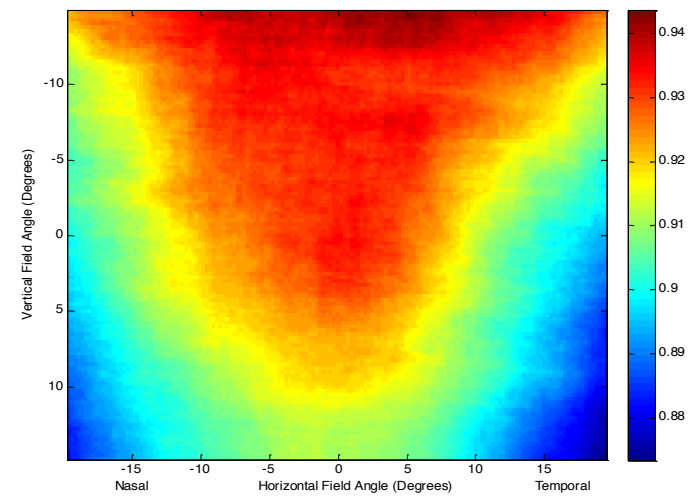

Fig. 3.20. Average retinal illuminance maps of all subjects combined.

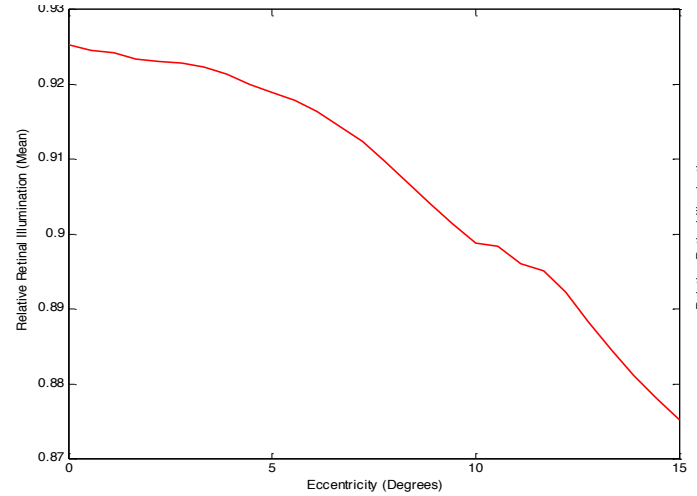

(a)

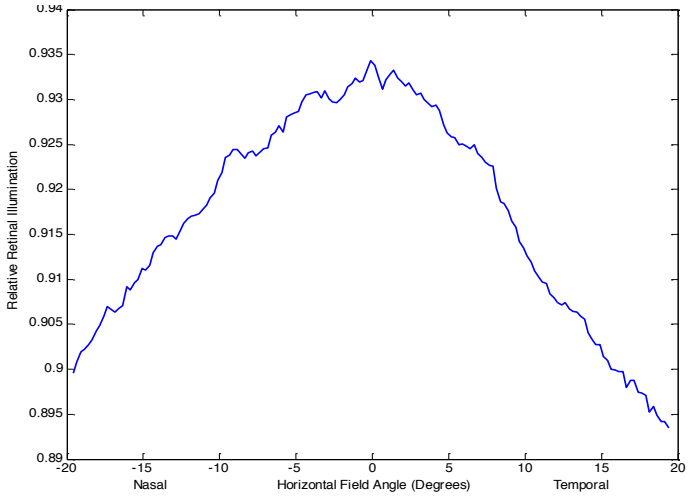

(b)

Fig. 3.21 (a) Mean retinal illumination versus eccentricity from Fig. 3.20. (b) Horizontal line-scan through the foveal region in Fig. 3.20.

Fig. 3.22 shows the average map of the results obtained for two of the experiments where the head of the subjects was immobilized (i.e. Phases 2 and 3). The map shows a central band of higher illumination and a clear maximum at the foveal center. Both the mean values and the horizontal linescan derived from this map showed a maximum at the center with a roughly uniform decrease in retinal illuminance with radial eccentricity from the fovea. 


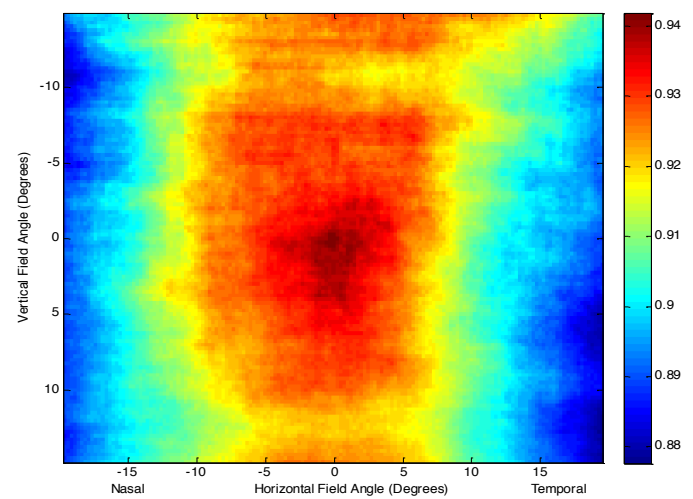

Fig. 3.22. Average map of the results obtained from two of the experiments in which the head of the subjects was immobilized (i.e. Phases 2 and 3).

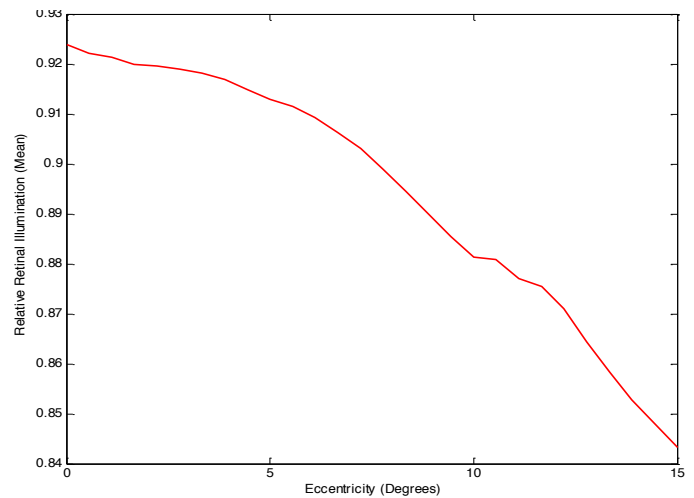

(a)

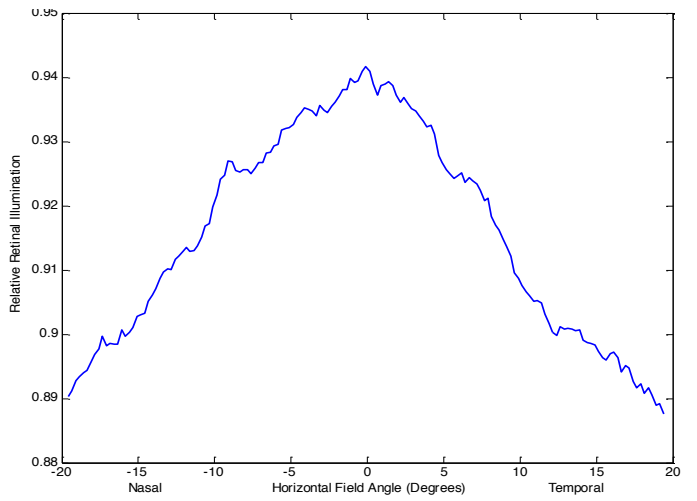

(b)

Fig. 3.23 (a) Mean retinal illumination versus eccentricity derived from Fig. 3.22. (b) Horizontal linescan through the foveal region derived from Fig. 3.22.

The average retinal illumination map obtained from subjects under free viewing conditions as they walked around the lab, hallways and campus is shown in Fig. 3.24. The map reveals an increase in illumination around the fovea but the maximum illumination is found away from the center. Both mean values and the horizontal line-scan derived from this map showed an almost constant retinal illumination. 


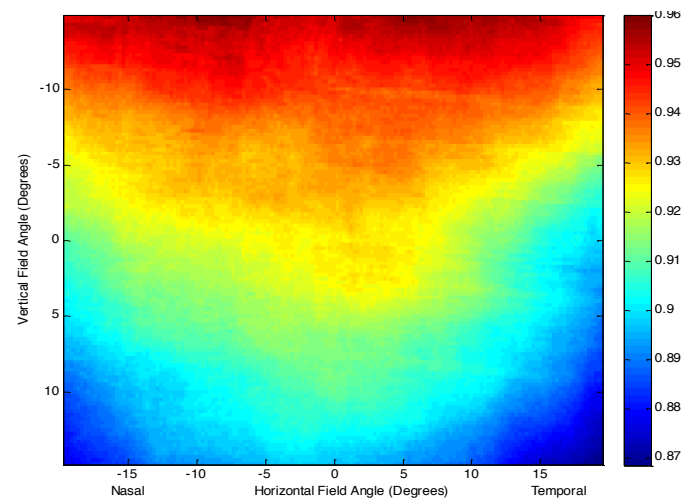

Fig. 3.24. Intensity maps (average) showing the cumulative light distribution (relative) on the retina for naïve subjects free viewing while walking around the lab, hallways and outside. (Phase 5).

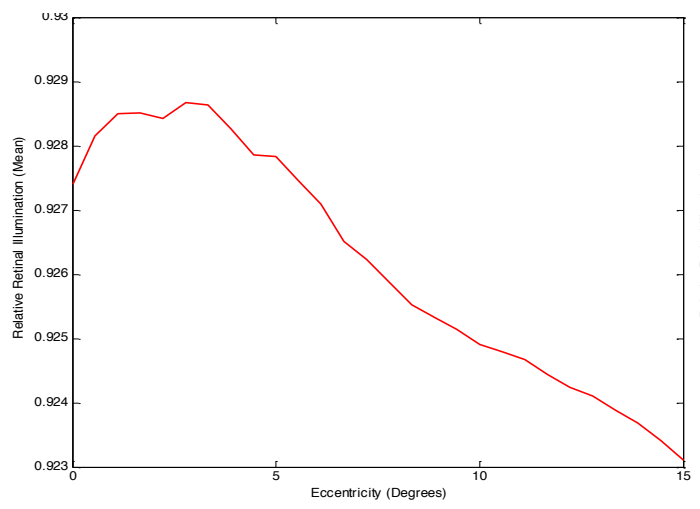

(a)

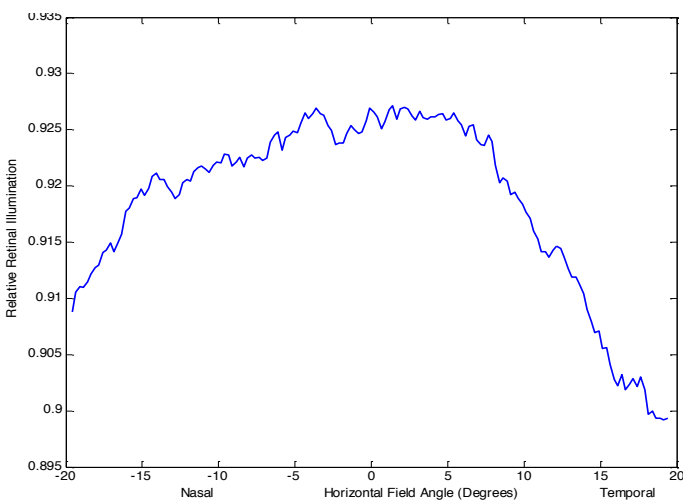

(b)

Fig. 3.25 (a) Mean retinal illumination versus eccentricity derived from Fig. 3.24. (b) Horizontal linescan through the foveal region derived from Fig. 3.24.

The results of Phase 1 were used to analyze the effect of age on the distribution of light in the retina. This phase was selected because there were 10 subjects under 21 years old $(17 \pm 2), 10$ subjects between the ages of 21 and $35(24 \pm 2)$, and 10 subjects over 35 years old $(45 \pm 7)$ and because Phase 1 did not include the scene camera as a photometer.

Fig. 3.26 shows the average map for young subjects $(<21$ years old $)$ viewing a gray-scale image (Phase 1). The map shows a broad area of higher illumination around the fovea with a maximum $4^{\circ}$ in the superior direction. The mean value versus eccentricity shows a maximum at the fovea and a relatively uniform decrease towards the periphery. The horizontal line-scan also peaks at the fovea and decreases in both the nasal and temporal directions. 


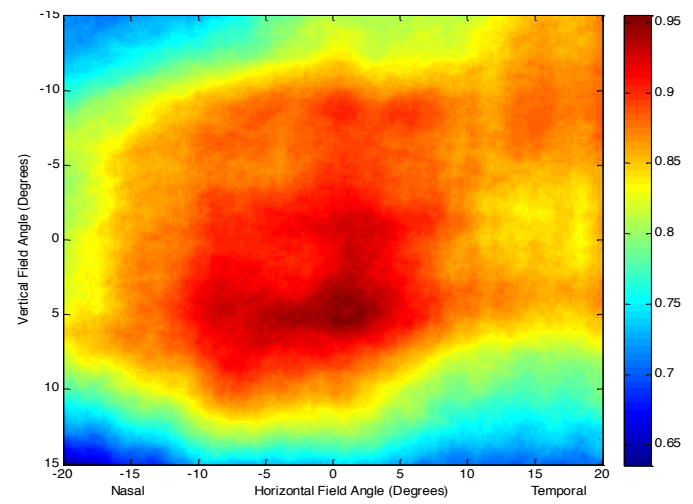

Fig. 3.26. Intensity maps (average) showing the cumulative light distribution (relative) on the retina for young subjects $(<21$ years old) viewing a gray scale image.

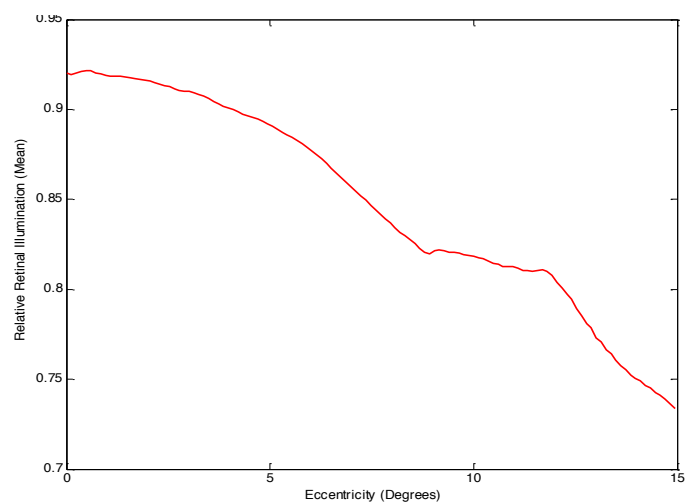

(a)

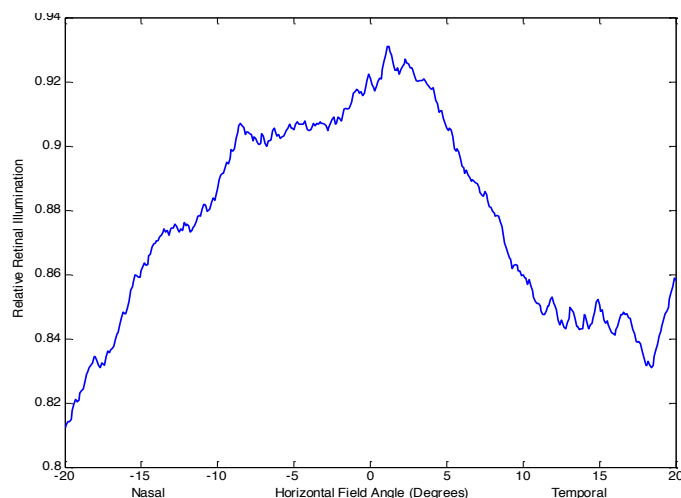

(b)

Fig. 3.27 (a) Mean retinal illumination versus eccentricity derived from Fig. 3.26. (b) Horizontal linescan through the foveal region derived from Fig. 3.26.

Fig. 3.28 shows the average map for subjects between the ages of 21 and 35 years old viewing a gray-scale image (Phase 1). The map shows a wide area of higher illumination in the nasal direction.

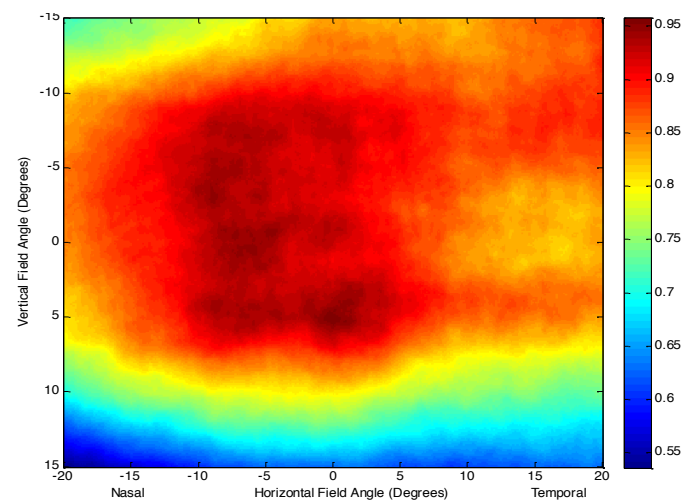

Fig. 3.28. Intensity maps (average) showing the cumulative light distribution (relative) on the retina for subjects between the ages of 21 and 35 years old viewing a gray scale image. 
The mean value versus eccentricity shows a maximum plateau around the fovea followed by a relatively uniform decrease with eccentricity. The horizontal line-scan shows a maximum $\sim 5^{\circ}$ in the nasal direction.

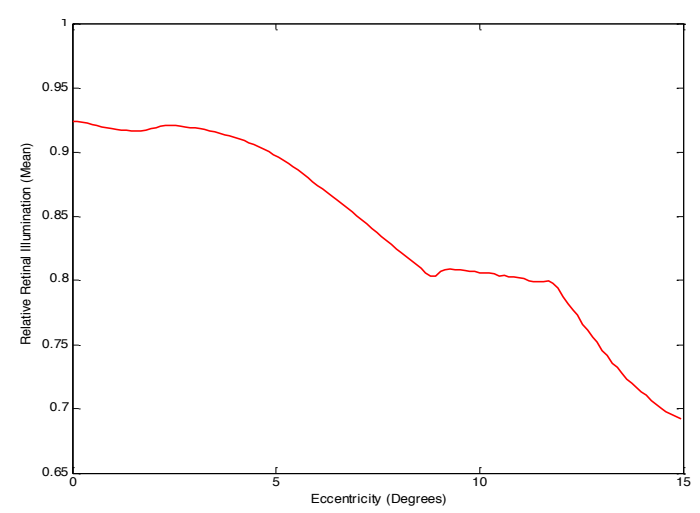

(a)

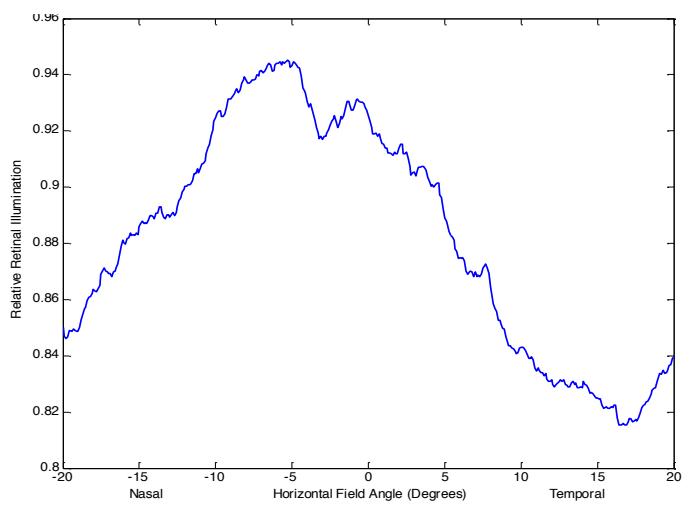

(b)

Fig. 3.29 (a) Mean retinal illumination versus eccentricity derived from Fig. 3.28. (b) Horizontal linescan through the foveal region derived from Fig. 3.28.

Fig. 3.30 shows the average map for subjects older than 35 viewing a gray-scale image. It shows a broad area of higher illumination with a well-defined peak in the nasal-superior direction. The mean value versus eccentricity plot was flat up to $\sim 5^{\circ}$ and then a $\sim 17 \%$ decrease with retinal eccentricity. The horizontal line-scan shows a maximum $\sim 6^{\circ}$ in the nasal direction.

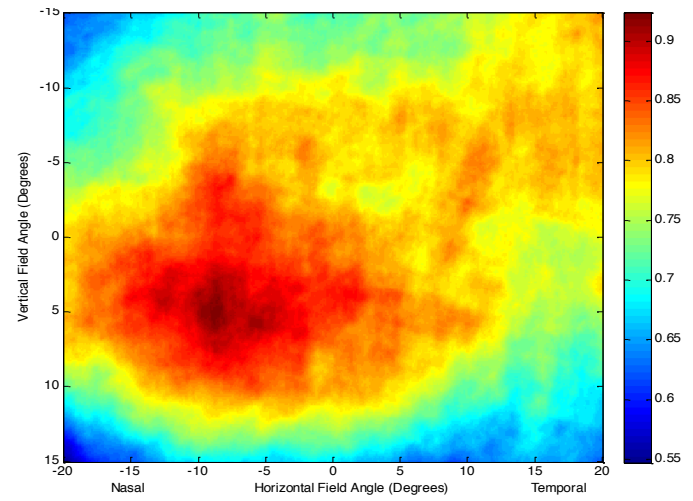

Fig. 3.30. Intensity maps (average) showing the cumulative light distribution (relative) on the retina for subjects older than 35 viewing a gray scale image. 


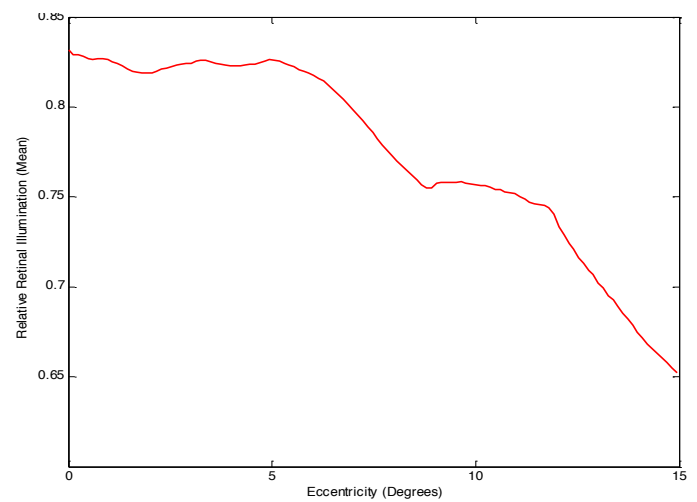

(a)

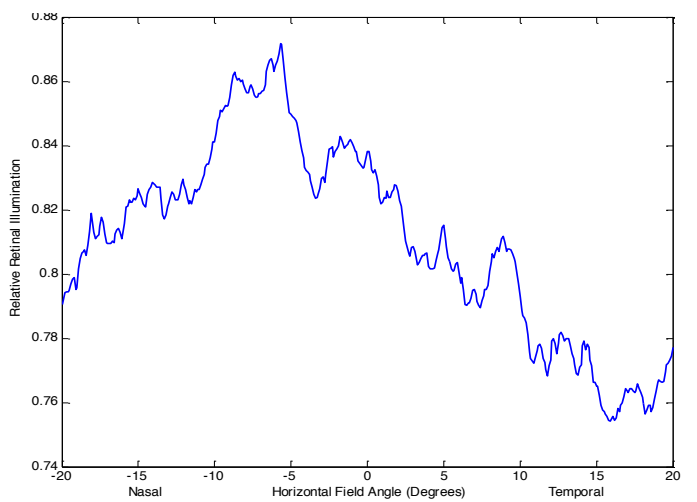

(b)

Fig. 3.31. Intensity maps (average) showing the cumulative light distribution (relative) on the retina for subjects older than 35 viewing a gray scale image.

Table 3.4 shows the analysis of the mean values of the difference in retinal illuminance within $1^{\circ}$ around the fovea and at $15^{\circ}$ of radial eccentricity. The highest difference was obtained for subjects between the ages of 21 and 35 years old. The results of the one-way ANOVA and the post hoc Turkey's test revealed no statistically significant difference among the age groups. See Tables 3.5 and 3.6.

\begin{tabular}{|c|c|c|c|c|c|c|c|c|}
\hline \multirow[t]{2}{*}{$\begin{array}{l}\text { Age } \\
\text { Group }\end{array}$} & \multirow[t]{2}{*}{$\mathrm{N}$} & \multirow[t]{2}{*}{ Mean } & \multirow[t]{2}{*}{$\begin{array}{c}\text { Std. } \\
\text { Deviation }\end{array}$} & \multirow[t]{2}{*}{$\begin{array}{l}\text { Std. } \\
\text { Error }\end{array}$} & \multicolumn{2}{|c|}{$\begin{array}{l}\text { 95\% Confidence Interval for } \\
\text { Mean } \\
\end{array}$} & \multirow[t]{2}{*}{ Minimum } & \multirow[t]{2}{*}{ Maximum } \\
\hline & & & & & Lower Bound & Upper Bound & & \\
\hline Under 21 & 10 & .1788 & .05982 & .01892 & .1360 & .2216 & .08 & .26 \\
\hline $21-35$ & 10 & .2215 & .01969 & .00623 & .2074 & .2356 & .19 & .26 \\
\hline Over 35 & 10 & .1664 & .06954 & .02199 & .1166 & .2161 & .08 & .27 \\
\hline Total & 30 & .1889 & .05752 & .01050 & .1674 & .2104 & .08 & .27 \\
\hline
\end{tabular}

Table 3.4. Analysis of the mean values of the difference in retinal illuminance within $1^{\circ}$ around the fovea and at $15^{\circ}$ of radial eccentricity for three age groups.

\begin{tabular}{|l|r|r|r|r|r|}
\hline & Sum of Squares & Df & Mean Square & F & Sig. \\
\hline Between Groups & .017 & 2 & .008 & 2.851 & .075 \\
Within Groups & .079 & 27 & .003 & & \\
Total & .096 & 29 & & & \\
\hline
\end{tabular}

Table 3.5. One-way ANOVA results for three age groups. 


\begin{tabular}{|ll|r|r|r|r|r|}
\hline $\begin{array}{l}\text { (I) Age } \\
\text { Group }\end{array}$ & (J) Age Group & $\begin{array}{r}\text { Mean Difference } \\
(\mathrm{I}-\mathrm{J})\end{array}$ & Std. Error & \multicolumn{1}{c|}{ Sig. } & \multicolumn{2}{|c|}{$95 \%$ Confidence Interval } \\
\cline { 6 - 7 } & & & & & Lower Bound & $\begin{array}{r}\text { Upper } \\
\text { Bound }\end{array}$ \\
\hline \multirow{2}{*}{ Under 21 } & $21-35$ & -.04272 & .02422 & .201 & -.1028 & .0173 \\
& Over 35 & .01242 & .02422 & .866 & -.0476 & .0725 \\
$21-35$ & Under 21 & .04272 & .02422 & .201 & -.0173 & .1028 \\
& Over 35 & .05514 & .02422 & .077 & -.0049 & .1152 \\
Over 35 & Under 21 & -.01242 & .02422 & .866 & -.0725 & .0476 \\
& $21-35$ & -.05514 & .02422 & .077 & -.1152 & .0049 \\
\hline
\end{tabular}

Table 3.6. Multiple comparison post hoc Turkey's test for three age groups. 


\section{DISCUSSION}

The results of this study demonstrate the feasibility of using an eye-tracker system to measure, at least semi-quantitatively, the distribution of light in the retina under natural viewing conditions, thus helping to understand the role played by ambient light exposure in the etiology of AMD. The most important limitation to this study lies in the fact that a CCD camera was used as a photometer to estimate the amount of light that reached the eye. Because of the noise and limited dynamic range of digital CCD cameras, nonlinear mapping algorithms have to be applied to the electrical response of the $\mathrm{CCD}$ before the image is transferred to a storage device such as a computer. At any given moment, a nonlinear relationship exists between the pixel value, I, in a frame recorded by the $\mathrm{CCD}$ camera and the actual intensity, $\mathrm{I}_{0}$, at the corresponding point in the scene being recorded. The simplest model relating I to $\mathrm{I}_{0}$ only takes into consideration the gamma correction of the camera (i.e. $\quad I=I_{0}^{\gamma}$ ) (Farid 2001) where $\gamma$ is a constant that represents the nonlinearity between $I$ and $I_{0}$ (Poynton 1996). However, the pixel values of the output image (or frame) depend on many other factors. Eq. 4.1 below describes the experimental model developed by Healey. (Healey and Kondepudy 1992). This model includes contributions from the dark current $\left(N_{D C}\right)$ (noise associate with the electrons created in the CCD by thermal energy), the shot noise $\left(N_{S}\right)$ (resulting from the discrete nature of the charge created in the CCD), the read-out noise $\left(N_{R}\right)$ (zero mean read noise created in the amplification of the CCD signal), the quantization noise $\left(N_{Q}\right)$ (resulting from the digitization of the analog signal coming from the camera), and the camera gain $(g)$.

$$
I=g\left(I_{0}+N_{D C}+N_{S}+N_{R}\right)+N_{Q} \quad \text { Eq. } 4.1
$$

If a gamma correction is applied to the CCD signal before digitalization (Withagen, Groen et al. 2005) Eq. 4.1 becomes

$$
I=g^{\gamma}\left(I_{0}+N_{D C}+N_{S}+N_{R}\right)^{\gamma}+N_{Q} \quad \text { Eq. } 4.2
$$


The most significant factor affecting the output of any CCD camera, and most importantly in a CCD camera with a small dynamic range, is the saturation point, where any pixel with intensity above a certain threshold is replaced by the same maximum value or saturation intensity. As a consequence, a pixel value in an image taken indoors under artificial illumination could the same as the pixel value of an image taken outside on a sunny day. This creates a problem that, in most cases, cannot be resolved, and the calibration of the radiometric response has to be done by comparative methods (Lin and Zhang 2005; Debevec and Malik 2008; Duan, Yan et al. 2013) or by statistical approximations (Tsin, Ramesh et al. 2001; Aguerrebere, Delon et al. 2012).

In this study, the visual fields provided illuminances that varied over several orders of magnitude (Sliney 1999; Bone, Gibert et al. 2012). However, the small dynamic rage of the scene camera, combined with the built-in gamma correction and automatic aperture, resulted in a compression of the upper ranges of illumination, and a limitation of the maximum pixel value to 255 (i.e. any scene luminance above the saturation point of the camera was assigned the same value of 255). For example, the pixel values measured by the scene camera of two different objects in the field of view under sunlight illumination were in a ratio of 5.5:1. However, when a calibrated, hand-held Minolta Spotmeter was used to measure the luminances of the same two objects under the same ambient light, they were in a ratio of $84: 1$. Therefore, any attempt to remove the gamma correction from the scene camera would not be accurate for pixel values close to, and including, 255. As a consequence, the results obtained for the variation in the spatial distribution of light on the retina for Phases 2, 3, 4 and 5, phases in which the scene camera was used as a photometer, are likely to be under-represented. Where these distributions indicate a small percentage increase in light intensity in the fovea compared with the periphery, the actual percentage increase is almost certainly considerably higher. For Phase 1, in which the subjects viewed a gray-scale image, gamma correction could not be removed because the original calibration function of the image was not available. 
Although the results of the comparison between the three viewing times showed no significant difference (See Tables 3.4, 3.5 and 3.6.), the accumulation and spatial distribution of light in the retina is known to be highly influenced by the exposure duration and by the intensity of the source (Delori, Webb et al. 2007). For example, for the same pupil diameter, fixating on the solar disk for $\sim 10^{-10} \mathrm{~s}$ results in the same cumulative foveal illuminance as that obtained by working on a computer for a day or two. In this test the subjects spent either 5, 10 or 15 minutes viewing a computer monitor and in each case, the relative light intensity distribution on the retina was essentially the same. In future studies, it would be interesting to repeat this type of comparison in situations where the visual scene is only available for a fleeting glance versus more prolonged viewing periods. In the former case, there might be a higher likelihood of the subject fixating on a bright object.

Considering the fact that in this study the spatial distributions of retinal illuminance are greatly under-represented, the results, at least partially, confirmed the hypothesis that, integrated over time, the distribution of light in the retina is greater at foveal locations. Excluding the results of Phase 4 (subjects viewing a computer monitor), $74 \%$ of the subjects showed an overall increase in retinal illuminance at or around the fovea (33\% with a sharp increase at the fovea, and $41 \%$ with a broader distribution around the fovea) and only $26 \%$ of the subjects showed a peak illuminance elsewhere in the retina. See Fig. 3.20. If the results of Phase 4 are included, then $77 \%$ of the subjects spent more time viewing brighter objects in the field of view (39\% showed an sharp increase at the fovea, $38 \%$ showed a broader increase in retinal illuminance around the fovea). Under restricted viewing conditions (i.e. the head of the subject was fixed), in general subjects spent more time fixating on bright features in the field of view. See Fig. 3.22. Under unrestricted conditions (i.e. subjects walking freely around the lab, hallways or around campus), the distribution of light on the retina showed maximum illumination in the inferior retina (see Fig. 3.24). These results are consistent with those of the Beaver Dam Study which found a much larger incidence of lens' opacity in the inferior nasal quadrant. (Klein, Klein et al. 1992). However, the findings of the current study differ from other geometrical assessments of retinal exposure that, based on restrictions imposed on the ambient light 
by the upper eye-lid, predict a higher illuminance in the superior retina. (Sliney 1999). With the eyetracker, it would be very difficult to mimic the effects of the upper eye-lid, or indeed the shielding of bright skylight in the upper visual field by an overhanging eyebrow.

Contrary to expectations, the one-way ANOVA analysis did not show a significant difference in the retinal illuminance between the three age groups. Because of the exponential increase of lens scattering with age (Michael and Bron 2011), one might think that people over 40 years old would be more comfortable fixating on bright objects as opposed to people younger than 21 . 


\section{CONCLUSIONS}

The main purpose of this study was to develop a method, based in an eye-tracking system, to quantify the distribution of light in on the retina under normal viewing conditions. The results of this study demonstrated the feasibility of developing such a method. These studies are innovative in that the cumulative spatial distribution of light on the retina is unknown, except under unnatural Ganzfeld illumination, and the use of an eye-tracking system for this purpose is completely novel. These results provide additional evidence for the role that light might play in the development of AMD. For the conditions under which the study was performed, the results partially support the original hypothesis that, integrated over time, the flux of light falling on the retina is greater in the central macular region than in the peripheral region. In particular there are some activities, such as using a computer, which consistently result in a peak in cumulative illuminance at the center of the retina. At the same time, this study has shown that some individuals spend more time fixating on bright objects in the field of view. For people for whom this is the case, or for those engaged for extended periods in those specific activities, there is the potential for an increased risk of AMD.

Further research is warranted in a number of areas. Extended study of those individuals who exhibited clear peaking of retinal illuminance at the fovea would indicate whether this was a consistent trend. (Funding restrictions in the current study imposed limits on subject involvement.) It would also be interesting to analyze the distribution of light on the retina in terms of different regions of the spectrum. This could easily be achieved if the color scene camera and image processing software permitted analysis of the camera's individual color channels, as was the case in an earlier study on the measurement of macular pigment using a retinal camera. (Bone, Brener, Gibert 2007). The rationale for such a study would be that the shorter wavelengths are more damaging to the retina than the longer, less energetic wavelengths. Iris color may also be a factor since light irises are less able to block ambient light, and might result in an aversion to bright light in those individuals. Likewise, those with incipient cataract or nuclear sclerosis might be more prone to glare disability resulting in a tendency to avoid looking directly at bright lights. It would also be interesting to 
compare retinal illuminance in right eye with the left eye. This could be attained by incorporating a binocular eye tracking system.

It would also be recommended to replace the scene camera by another with a fixed gain control, a larger dynamic range and a known gamma function. With such a system, absolute photometric measurements should be possible. Improvement of the head-to-eye tracker attachment would reduce the potential problem of slip allowing for better calibration and more reliable measurements. The slip problem with the current system was more likely to occur in Phase 5 when the subjects were walking freely.

While the work described in this dissertation represents an initial feasibility study, it paves the way for exploring retinal light distributions during a variety of viewing activities such as driving, shopping, or what appears to be fast becoming the most prolific human activity, using a smart phone. 


\section{REFERENCES}

Age-Related, Eye Disease Study Research Group (2000). Risk factors associated with age-related macular degeneration. A case-control study in the age-related eye disease study. Age-Related Eye Disease Study Report Number 3. Ophthalmology, 107(12), 2224-2232.

Aguerrebere, C., Delon, J., Gousseau, Y., \& Musé, P. (2012). Study of the digital camera acquisition process and statistical modeling of the sensor raw data. At http://hal.archives-ouvertes.fr/hal00733538 .

Alió, J. L., Schimchak, P., Negri, H. P., \& Montés-Micó, R. (2005). Crystalline lens optical dysfunction through aging. Ophthalmology, 112(11), 2022-2029.

Eye Disease Case-Control Study Group (1993) Antioxidant status and neovascular age-related macular degeneration. Archives of ophthalmology 111: 104-9.

Bird, A. C., Bressler, N. M., Bressler, S. B., Chisholm, I. H., Coscas, G., Davis, M. D., Vingerling, J. R. (1995). An international classification and grading system for age-related maculopathy and age-related macular degeneration. Survey of ophthalmology, 39(5), 367374.

Bone, R. A., \& Landrum, J. T. (1984). Macular pigment in Henle fiber membranes: a model for Haidinger's brushes. Vision research, 24(2), 103-108.

Bone, R. A., Brener, B., \& Gibert, J. C. (2007). Macular pigment, photopigments, and melanin: distributions in young subjects determined by four-wavelength reflectometry. Vision research, 47(26), 3259-3268.

Bone, R. A., Gibert, J. C., \& Mukherjee, A. (2012). Light distributions on the retina: relevance to macular pigment photoprotection. Acta Biochimica Polonica, 59(1), 91-96.

Bone, R. A., Landrum, J. T., Fernandez, L., \& Tarsis, S. L. (1988). Analysis of the macular pigment by HPLC: retinal distribution and age study. Investigative ophthalmology \& visual science, 29(6), 843-849.

Bone, R. A., Landrum, J. T., Friedes, L. M., Gomez C. M., Kilburn, M. D., Menendez, E.,\& Wang, W. (1997). Distribution of lutein and zeaxanthin stereoisomers in the human retina. Experimental eye research, 64(2), 211-218.

Bone, R. A., Landrum, J. T., Mayne, S. T., Gomez, C. M., Tibor, S. E., \& Twaroska, E. E. (2001). Macular pigment in donor eyes with and without AMD: a case-control study. Investigative ophthalmology \& visual science, 42(1), 235-240.

Borja, D., Manns, F., Ho, A., Ziebarth, N., Rosen, A. M., Jain, R., ... \& Parel, J. M. (2008). Optical power of the isolated human crystalline lens. Investigative ophthalmology \& visual science, 49(6), 2541-2548.

Bour, L. J., Koo, L., Delori, F. C., Apkarian, P., \& Fulton, A. B. (2002). Fundus photography for measurement of macular pigment density distribution in children. Investigative ophthalmology \& visual science, 43(5), 1450-1455. 
Campbell, F. W., \& Gubisch, R. W. (1966). Optical quality of the human eye. The Journal of Physiology, 186(3), 558-578.

Casten, R., \& Rovner, B. (2008). Depression in age-related macular degeneration. Journal of visual impairment \& blindness, 102(10), 591.

Chakravarthy, U., Wong, T., Fletcher, A., Piault, E., Evans, C., Zlateva, G., Mitchell, P. (2010). Clinical risk factors for age-related macular degeneration: a systematic review and metaanalysis. BMC ophthalmology, 10(1), 31.

Clarkson, T. G. (1989). "Safety aspects in the use of infrared detection systems". I. J. Electronics, 66, 6 929-934.

Cruickshanks KJ, Klein R, Klein BEK (1993). Sunlight and age-related macular degeneration. Archives of ophthalmology 111: 514-518.

Curcio, C. A., Millican, C. L., Allen, K. A., \& Kalina, R. E. (1993). Aging of the human photoreceptor mosaic: evidence for selective vulnerability of rods in central retina. Investigative ophthalmology \& visual science, 34(12), 3278-3296.

Darzins P, Mitchell P, Heller RF (1997). Sun exposure and age-related macular degeneration: An Australian case-control study. Ophthalmology 104: 770-776.

Debevec, P. E., \& Malik, J. (2008, August). Recovering high dynamic range radiance maps from photographs. In ACM SIGGRAPH 2008 classes (p. 31). ACM.

Delcourt C, Carrière I, Ponton-Sanchez A, Fourrey S, Lacroux A, Papoz L (2001). Light exposure and the risk of age-related macular degeneration. The Pathologies Oculaires Liées à l'Age (POLA) Study. Archives of ophthalmology 119(10): 1463-1468.

Delori, F. C., \& Burns, S. A. (1996). Fundus reflectance and the measurement of crystalline lens density. JOSA A, 13(2), 215-226.

Delori, F. C., \& Pflibsen, K. P. (1989). Spectral reflectance of the human ocular fundus. Applied Optics, 28(6), 1061-1077.

Delori, F. C., Goger, D. G., Hammond, B. R., Snodderly, D. M., \& Burns, S. A. (2001). Macular pigment density measured by autofluorescence spectrometry: comparison with reflectometry and heterochromatic flicker photometry. JOSA, 18(6), 1212-1230.

Delori, F. C., Webb, R. H., \& Sliney, D. H. (2007). Maximum permissible exposures for ocular safety (ANSI 2000), with emphasis on ophthalmic devices. JOSA A, 24(5), 1250-1265.

Donnelly III, W. J., \& Roorda, A. (2003). Optimal pupil size in the human eye for axial resolution. JOSA A, 20(11), 2010-2015.

Dorey, C. K., Wu, G., Ebenstein, D., Garsd, A., \& Weiter, J. J. (1989). Cell loss in the aging retina. Relationship to lipofuscin accumulation and macular degeneration. Investigative ophthalmology \& visual science, 30(8), 1691-1699.

Duan, Y., Yan, L., Yang, B., Jing, X., \& Chen, W. (2013). Outdoor relative radiometric calibration method using gray scale targets. Science China Technological Sciences, 1-10. 
Evans JR (2001) Risk factors for age-related macular degeneration Prog Retin Eye Res 20: 227-53.

Eye Disease Case-Control Study Group (1992). Risk factors for age-related macular degeneration. Archives of ophthalmology 110: 1701-1708.

EyeTracker, V. (2005). PC-60 Scene Camera Option Users Guide. Scottsdale, AZ.

Farid, H. (2001). Blind inverse gamma correction. Image Processing, IEEE Transactions on, 10(10), 1428-1433.

Forrester, J. V., Dick, A. D., McMenamin, P. G., \& Lee, W. R. (2002). The Eye: Basic Sciences in Practice (WB Saunders).

Friedman DS, BJ O'Colmain, B Munoz, SC Tomany, C McCarty, et al. (2004) Prevalence of agerelated macular degeneration in the United States Archives of ophthalmology 122: 564-72.

Garner, L. F., Ooi, C. S., \& Smith, G. (1998). Refractive index of the crystalline lens in young and aged eyes. Clinical and Experimental Optometry, 81(4), 145-150.

Gosbell, A. D., Stefanovic, N., Scurr, L. L., Pete, J., Kola, I., Favilla, I., \& de Haan, J. B. (2006). Retinal light damage: structural and functional effects of the antioxidant glutathione peroxidase-1. Investigative ophthalmology \& visual science, 47(6), 2613-2622.

Gottsch, J. D., Pou, S., Bynoe, L. A., \& Rosen, G. M. (1990). Hematogenous photosensitization. A mechanism for the development of age-related macular degeneration. Investigative ophthalmology \& visual science, 31(9), 1674-1682.

Gowdy, P. D., \& Cicerone, C. M. (1998). The spatial arrangement of the L and M cones in the central fovea of the living human eye. Vision research, 38(17), 2575-2589.

Guide, M. U. S. (1998). The mathworks. Inc., Natick, MA, 5.

Haegerstrom-Portnoy, G. (1988). Short wavelength-sensitive-cone sensitivity loss with aging: a protective role for macular pigment?. JOSA A, 5(12), 2140-2144.

Hammond Jr, B. R., Curran-Celentano, J., Judd, S., Fuld, K., Krinsky, N. I., Wooten, B. R., \& Snodderly, D. (1996). Sex Differences in Macular Pigment Optical Density:: Relation to Plasma Carotenoid Concentrations and Dietary Patterns. Vision research, 36(13), 2001-2012.

Hayman, K. J., Kerse, N. M., La Grow, S. J., Wouldes, T., Robertson, M. C., \& Campbell, A. J. (2007). Depression in older people: visual impairment and subjective ratings of health. Optometry \& Vision Science, 84(11), 1024-1030.

Healey, G., \& Kondepudy, R. (1992, June). CCD camera calibration and noise estimation. In Computer Vision and Pattern Recognition, 1992. Proceedings CVPR'92., 1992 IEEE Computer Society Conference on (pp. 90-95). IEEE.

Hemenger, R. P., Garner, L. F., \& Ooi, C. S. (1995). Change with age of the refractive index gradient of the human ocular lens. Investigative ophthalmology \& visual science, 36(3), 703-707.

Howland, H. C., \& Howland, B. (1977). A subjective method for the measurement of monochromatic aberrations of the eye. JOSA, 67(11), 1508-1518. 
Hunold, W., \& Malessa, P. (1974). Spectrophotometric determination of the melanin pigmentation of the human ocular fundus in vivo. Ophthalmic Research, 6(5-6), 355-362.

Hyman L. (1992) Epidemiology of AMD. In Age-Related Macular Degeneration: Principles and Practice. Eds Hampton GR, Nelson PT. Raven Press, New York.

Jager RD, WF Mieler, JW Miller (2008) Age-related macular degeneration. N Engl J Med 358: 260617.

Kasthurirangan, S., Markwell, E. L., Atchison, D. A., \& Pope, J. M. (2008). In vivo study of changes in refractive index distribution in the human crystalline lens with age and accommodation. Investigative ophthalmology \& visual science, 49(6), 2531-2540.

Keeley, F. W., Morin, J. D., \& Vesely, S. (1984). Characterization of collagen from normal human sclera. Experimental eye research, 39(5), 533-542.

Kirkness, C. M. (1986). Do ophthalmic instruments pose a hazard of light-induced damage to the eye. Hazards of Light, Myths and Realities, Eye and Skin. Oxford: Pergamon.

Kirschfeld, K. (1982). Carotenoid pigments: their possible role in protecting against photooxidation in eyes and photoreceptor cells. Proceedings of the Royal Society of London. Series B. Biological Sciences, 216(1202), 71-85.

Klein, B. E., Klein, R., \& Linton, K. L. (1992). Prevalence of age-related lens opacities in a population. The Beaver Dam Eye Study. Ophthalmology, 99(4), 546-552.

Klein, R., Klein, B. E., Linton, K. L., \& DeMets, D. L. (1993). The Beaver Dam Eye Study: the relation of age-related maculopathy to smoking. American journal of epidemiology, 137(2), 190-200.

Knighton, R. W., Jacobson, S. G., \& Roman, M. I. (1989, September). Specular reflection from the surface of the retina. In OE/LASE'89, 15-20 Jan., Los Angeles. CA (pp. 10-17). International Society for Optics and Photonics.

Kooijman, A. C. (1983). Light distribution on the retina of a wide-angle theoretical eye. JOSA, 73(11), 1544-1550.

Landrum, J. T., Bone, R. A., \& Kilburn, M. D. (1996). The macular pigment: a possible role in protection from age-related macular degeneration. Advances in pharmacology, 38, 537-556.

Lefohn, A., Budge, B., Shirley, P., Caruso, R., \& Reinhard, E. (2003). An ocularist's approach to human iris synthesis. Computer Graphics and Applications, IEEE, 23(6), 70-75.

Li, B., Ahmed, F., \& Bernstein, P. S. (2010). Studies on the singlet oxygen scavenging mechanism of human macular pigment. Archives of biochemistry and biophysics, 504(1), 56-60.

Lin, S., \& Zhang, L. (2005, June). Determining the radiometric response function from a single grayscale image. In Computer Vision and Pattern Recognition, 2005. CVPR 2005. IEEE Computer Society Conference on (Vol. 2, pp. 66-73). IEEE. 
Margrain, T. H., Boulton, M., Marshall, J., \& Sliney, D. H. (2004). Do blue light filters confer protection against age-related macular degeneration?. Progress in retinal and eye research, 23(5), 523-531.

McCarty, C. A., Mukesh, B. N., Fu, C. L., Mitchell, P., Wang, J. J., \& Taylor, H. R. (2001). Risk factors for age-related maculopathy: the Visual Impairment Project. Archives of ophthalmology, 119(10), 1455.

Meral, İ., \& Bilgili, Y. (2011). Diffusion Changes in the Vitreous Humor of the Eye during Aging. American Journal of Neuroradiology, 32(8), 1563-1566.

Michael, R., \& Bron, A. J. (2011). The ageing lens and cataract: a model of normal and pathological ageing. Philosophical Transactions of the Royal Society B: Biological Sciences, 366(1568), 1278-1292.

Mitchell P, Smith W, Wang JJ (1998). Iris color, skin sun sensitivity, and age-related maculopathy: The Blue Mountains eye study. Ophthalmology 105: 1359-1363.

Nickerson, C. S. (2006). Engineering the mechanical properties of ocular tissues (Doctoral dissertation, California Institute of Technology).

Norren, D. V., \& Vos, J. J. (1974). Spectral transmission of the human ocular media. Vision Research, 14(11), 1237-1244.

Osterberg, G. (1935). Topography of the layer of rods and cones in the human retina. Acta Ophthalmol., Suppl. 13:6, pp. 1-102

Pajoohesh-Ganji, A., \& Stepp, M. A. (2005). In search of markers for the stem cells of the corneal epithelium. Biology of the Cell, 97(4), 265-276.

Pflibsen, K. P., Pomerantzeff, O., \& Ross, R. N. (1988). Retinal illuminance using a wide-angle model of the eye. JOSA A, 5(1), 146-150.

Pokorny, J., Smith, V. C., \& Lutze, M. (1987). Aging of the human lens. Applied Optics, 26(8), 14371440 .

Polyak, S. L. (1941). The retina: the anatomy and the histology of the retina in man, ape, and monkey, including the consideration of visual functions, the history of physiological optics, and the histological laboratory technique. Chicago: University of Chicago Press.

Poynton, C. A. (1996). A technical introduction to digital video. John Wiley \& Sons, Inc..

Reading, V. M., \& Weale, R. A. (1974). Macular pigment and chromatic aberration. JOSA, 64(2), 231-234.

Resnikoff, S., Pascolini, D., Etya'ale, D., Kocur, I., Pararajasegaram, R., Pokharel, G. P., \& Mariotti, S. P. (2004). Global data on visual impairment in the year 2002. Bulletin of the World Health Organization, 82(11), 844-851.

Roorda, A., Metha, A. B., Lennie, P., \& Williams, D. R. (2001). Packing arrangement of the three cone classes in primate retina. Vision research, 41(10), 1291-1306. 
Rovner BW, RJ Casten (2002) Activity loss and depression in age-related macular degeneration Am J Geriatr Psychiatry 10: 305-10

Sebag, J., Buckingham, B., Charles, M. A., \& Reiser, K. (1992). Biochemical abnormalities in vitreous of humans with proliferative diabetic retinopathy. Archives of ophthalmology, 110(10), 1472.

Sliney, D. H. (1999). Geometrical assessment of ocular exposure to environmental UV radiation-implications for ophthalmic epidemiology. Journal of epidemiology/Japan Epidemiological Association, 9(6 Suppl), S22.

Sliney, D., Aron-Rosa, D., DeLori, F., Fankhauser, F., Landry, R., Mainster, M., ... \& Wolffe, M. (2005). Adjustment of guidelines for exposure of the eye to optical radiation from ocular instruments: statement from a task group of the International Commission on Non-Ionizing Radiation Protection (ICNIRP). Applied optics, 44(11), 2162-2176.

Snodderly, D. M. (1995). Evidence for protection against age-related macular degeneration by carotenoids and antioxidant vitamins. The American journal of clinical nutrition, 62(6), 1448S-1461S.

Snodderly, D. M., Auran, J. D., \& Delori, F. C. (1984). The macular pigment. II. Spatial distribution in primate retinas. Investigative ophthalmology \& visual science, 25(6), 674-685.

Sui, G. Y., Liu, G. C., Liu, G. Y., Gao, Y. Y., Deng, Y., Wang, W. Y., ... \& Wang, L. (2013). Is sunlight exposure a risk factor for age-related macular degeneration? A systematic review and meta- analysis. British Journal of Ophthalmology, 97(4), 389-394.

Taylor H, West S, Munoz B, Rosenthal F, Bressler S, Bressler N (1992). The long-term effects of visible light on the eye. Archives of ophthalmology 110: 99-104.

The Age-Related Eye Disease Study Group (2000) Risk factors associated with age-related macular degeneration. A case-control study in the age-related eye disease study: Age-Related Eye Disease Study Report Number 3 Ophthalmology 107: 2224-32.

Thornton, J., Edwards, R., Mitchell, P., Harrison, R. A., Buchan, I., \& Kelly, S. P. (2005). Smoking and age-related macular degeneration: a review of association. Eye, 19(9), 935-944.

Tsin, Y., Ramesh, V., \& Kanade, T. (2001). Statistical calibration of CCD imaging process. In Computer Vision, 2001. ICCV 2001. Proceedings. Eighth IEEE International Conference on (Vol. 1, pp. 480-487). IEEE.

Van de Kraats, J., Berendschot, T. T., \& Van Norren, D. (1996). The pathways of light measured in fundus reflectometry. Vision research, 36(15), 2229-2247.

Van Norren, D., \& Tiemeijer, L. F. (1986). Spectral reflectance of the human eye. Vision research, 26(2), 313-320.

Vojniković B, Njirić S, Coklo M, Spanjol J (2007). Ultraviolet sun radiation and incidence of agerelated macular degeneration on Croatian Island of Rab. Coll Antropol 31 (Suppl 1): 43-44.

Wald, G. (1945). Human vision and the spectrum. Science. 101:653-58 
Weale, R. A. (1988). Age and the transmittance of the human crystalline lens. The Journal of Physiology, 395(1), 577-587.

Withagen, P. J., Groen, F. C., \& Schutte, K. (2005, May). CCD characterization for a range of color cameras. In Instrumentation and Measurement Technology Conference, 2005. IMTC 2005. Proceedings of the IEEE, Vol. 3, pp. 2232-2235

Yu, W. (2004). Practical anti-vignetting methods for digital cameras. Consumer Electronics, IEEE Transactions on, 50(4), 975-983. 


\section{APPENDIX 1}

This appendix contains the series of photographic images presented to the subjects in Phase 2. A blank screen (not shown) was inserted in between each image.
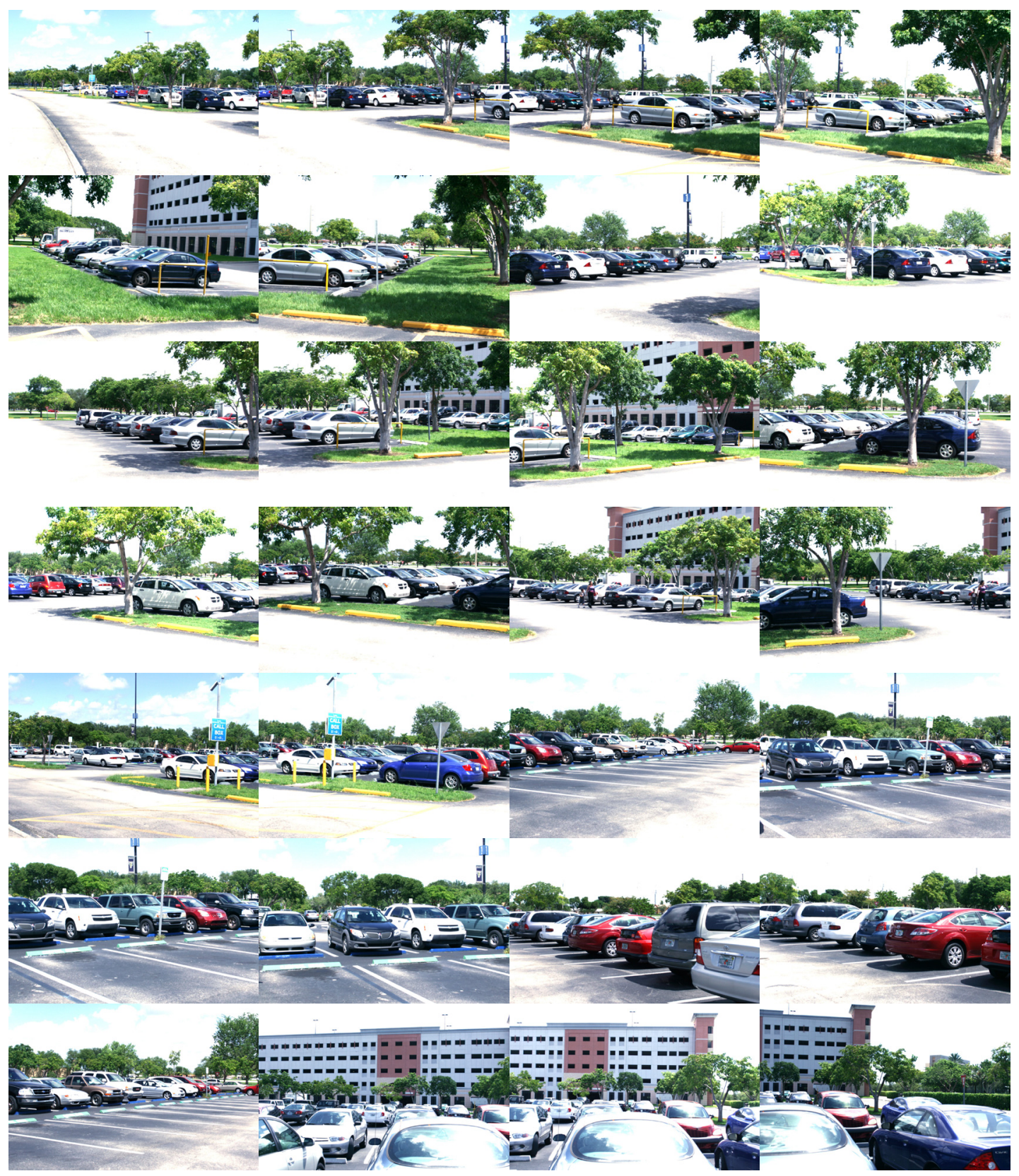


\section{APPENDIX 2}

\section{This appendix contains the MATLAB program used to process the data from Phase 1}

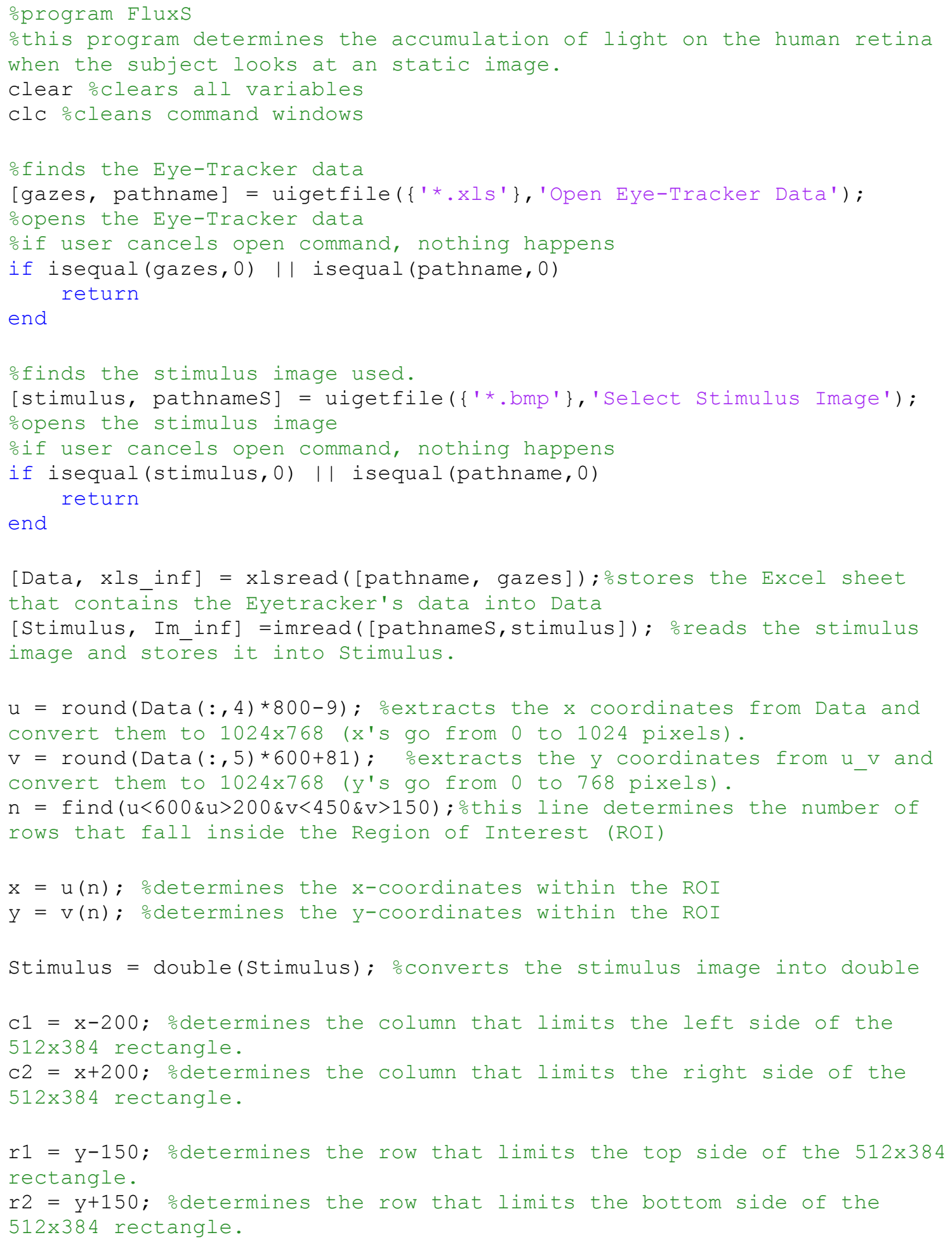




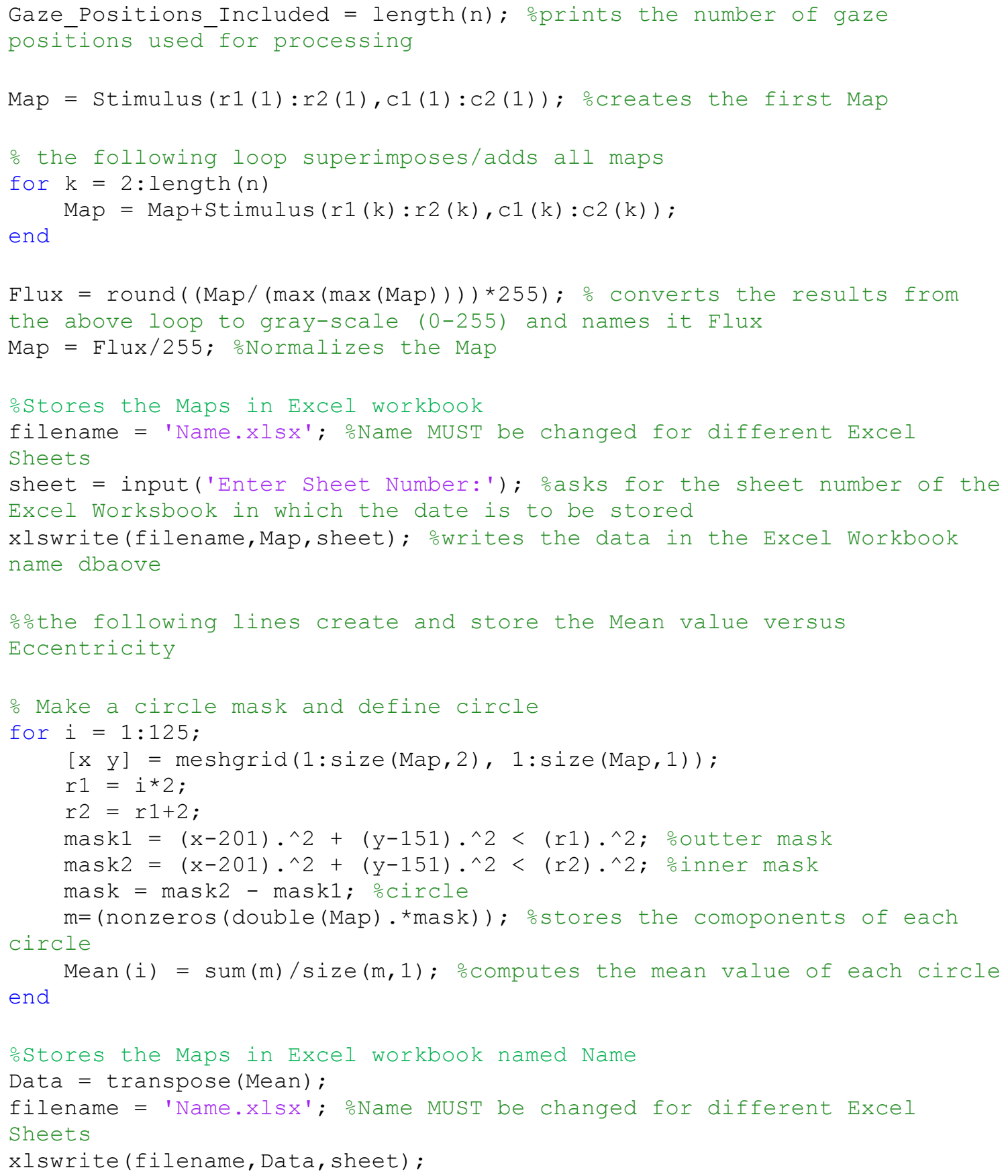




\section{APPENDIX 3}

This appendix contains the MATLAB program used to process the data from Phases 2, 3, 4, and 5

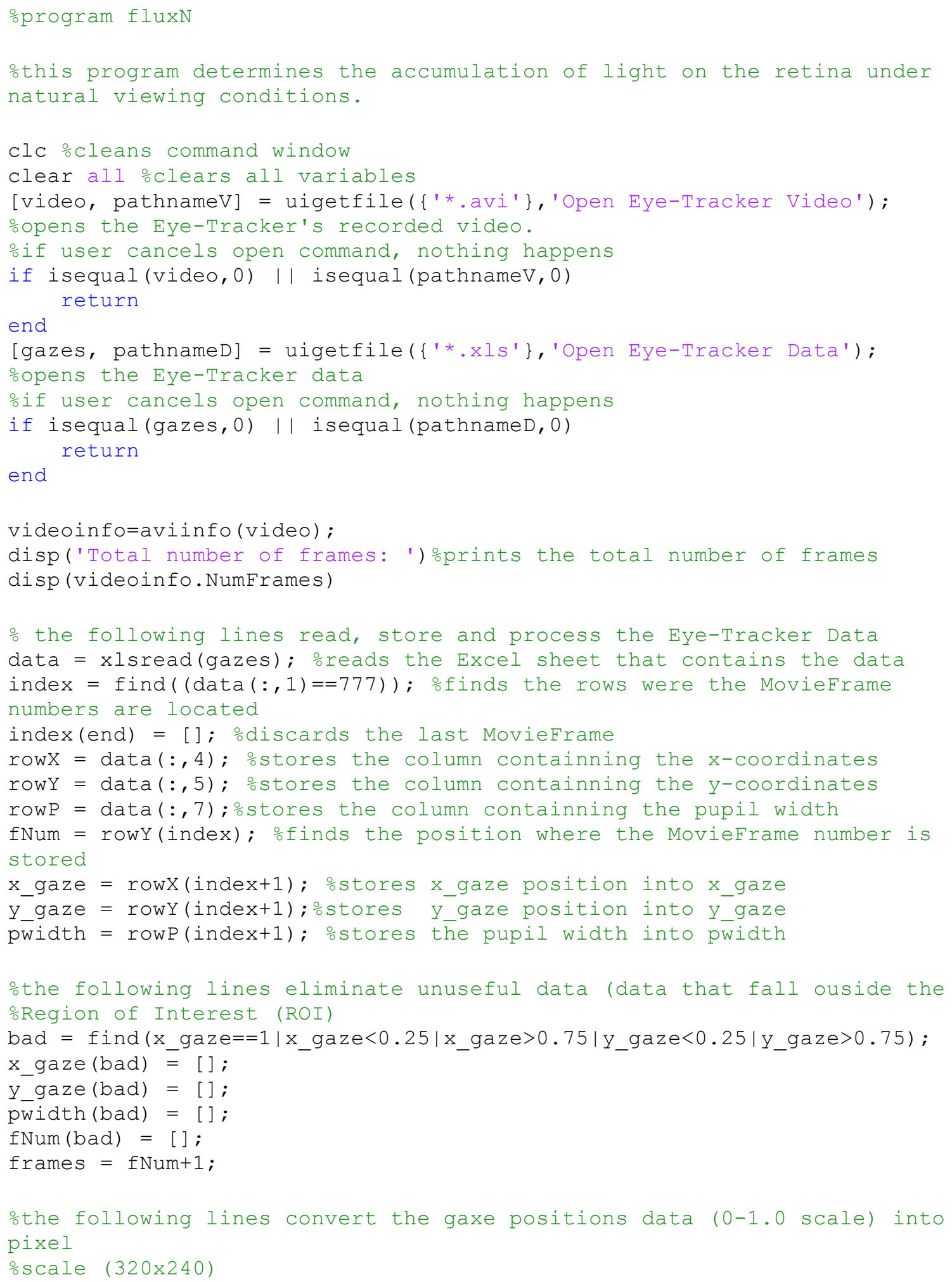




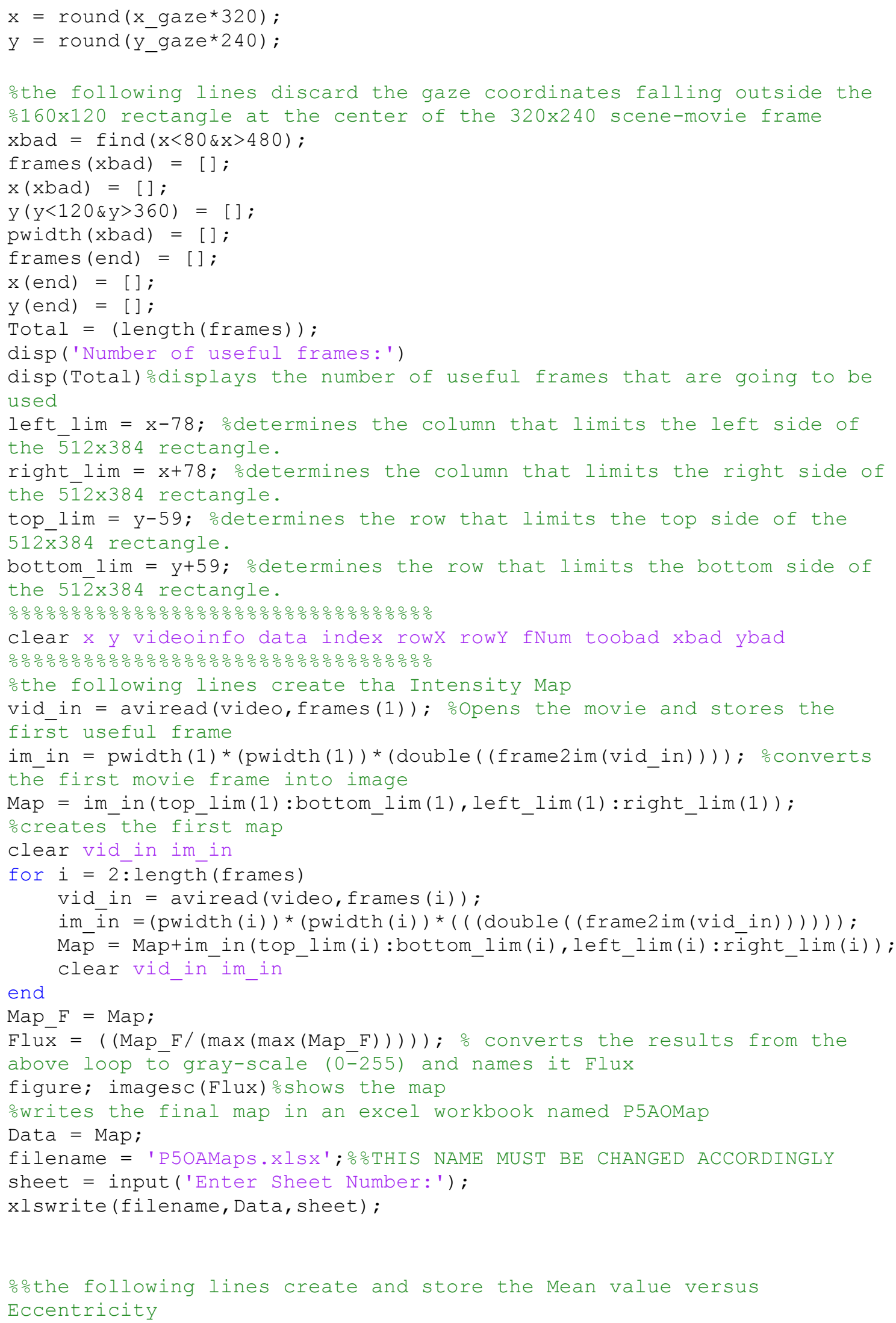




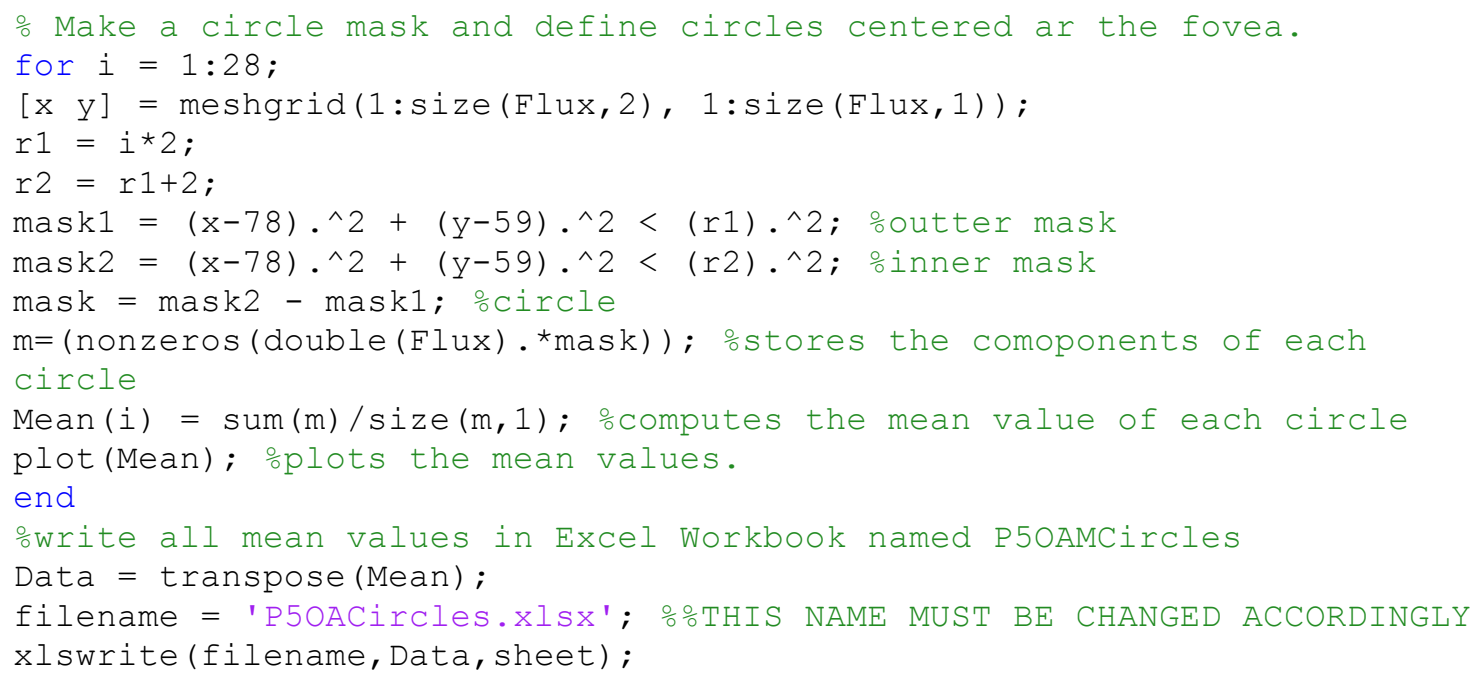




\section{APPENDIX 4}

This appendix contains the MATLAB program used to plot the illumination maps, the horizontal and vertical line-scans across the fovea, and the retinal illumination versus radial eccentricity from the fovea.

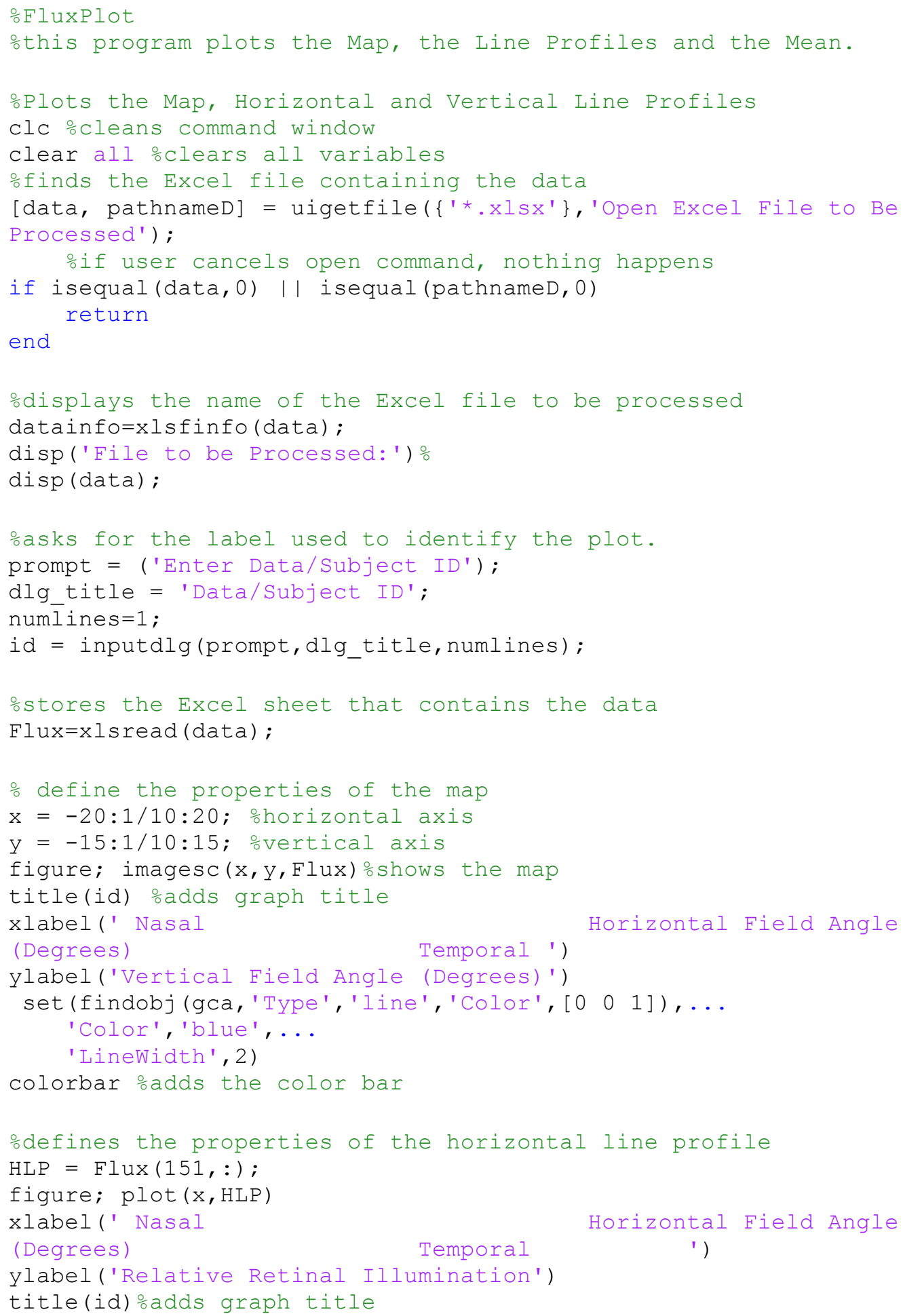




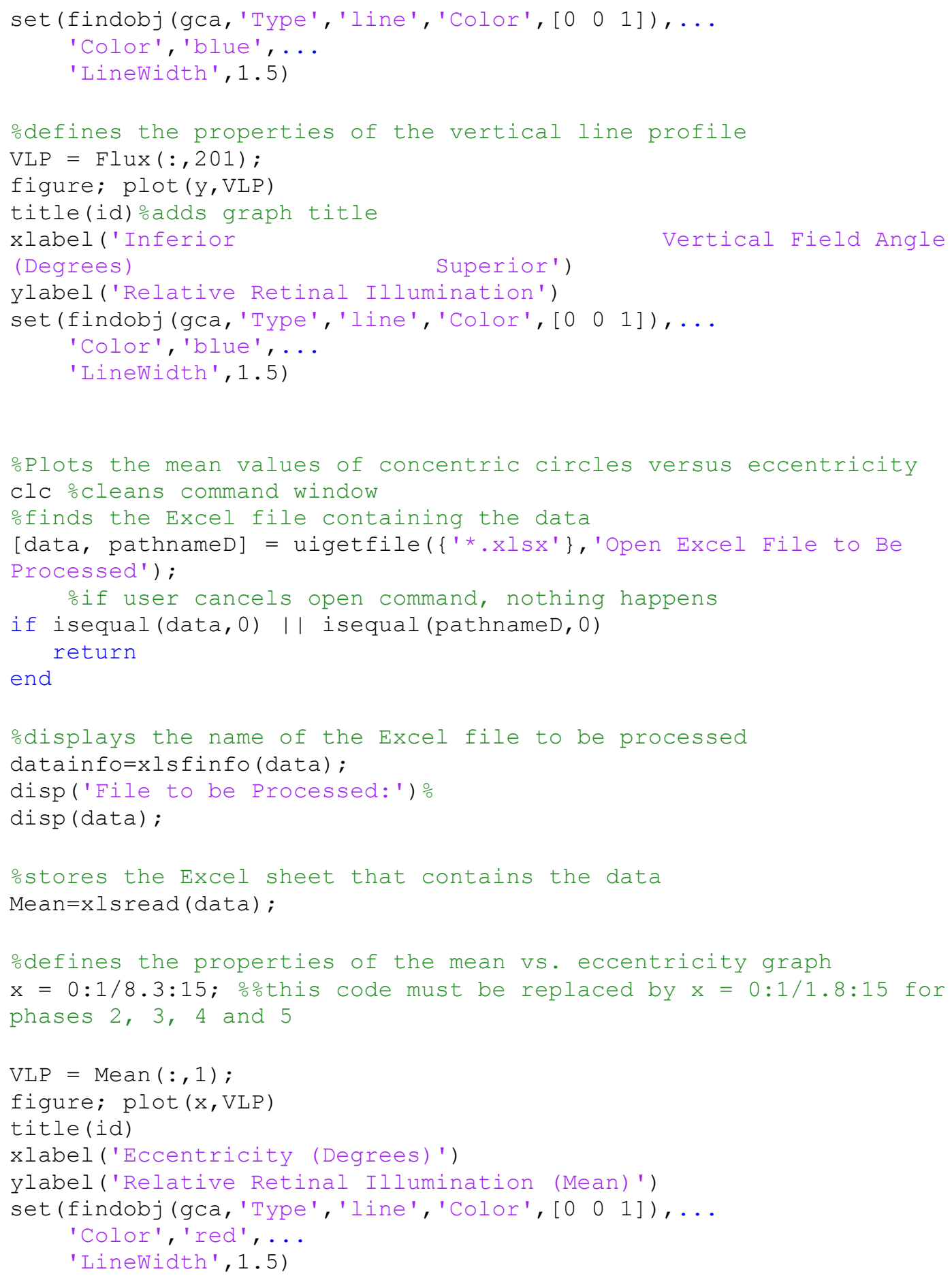




\section{APPENDIX 5}

This appendix contains the individual retinal illumination maps, the horizontal and vertical line-scans across the fovea, and the retinal illumination versus radial eccentricity from the fovea for the subjects under 21 years old that participated in Phase 1. (P1A)

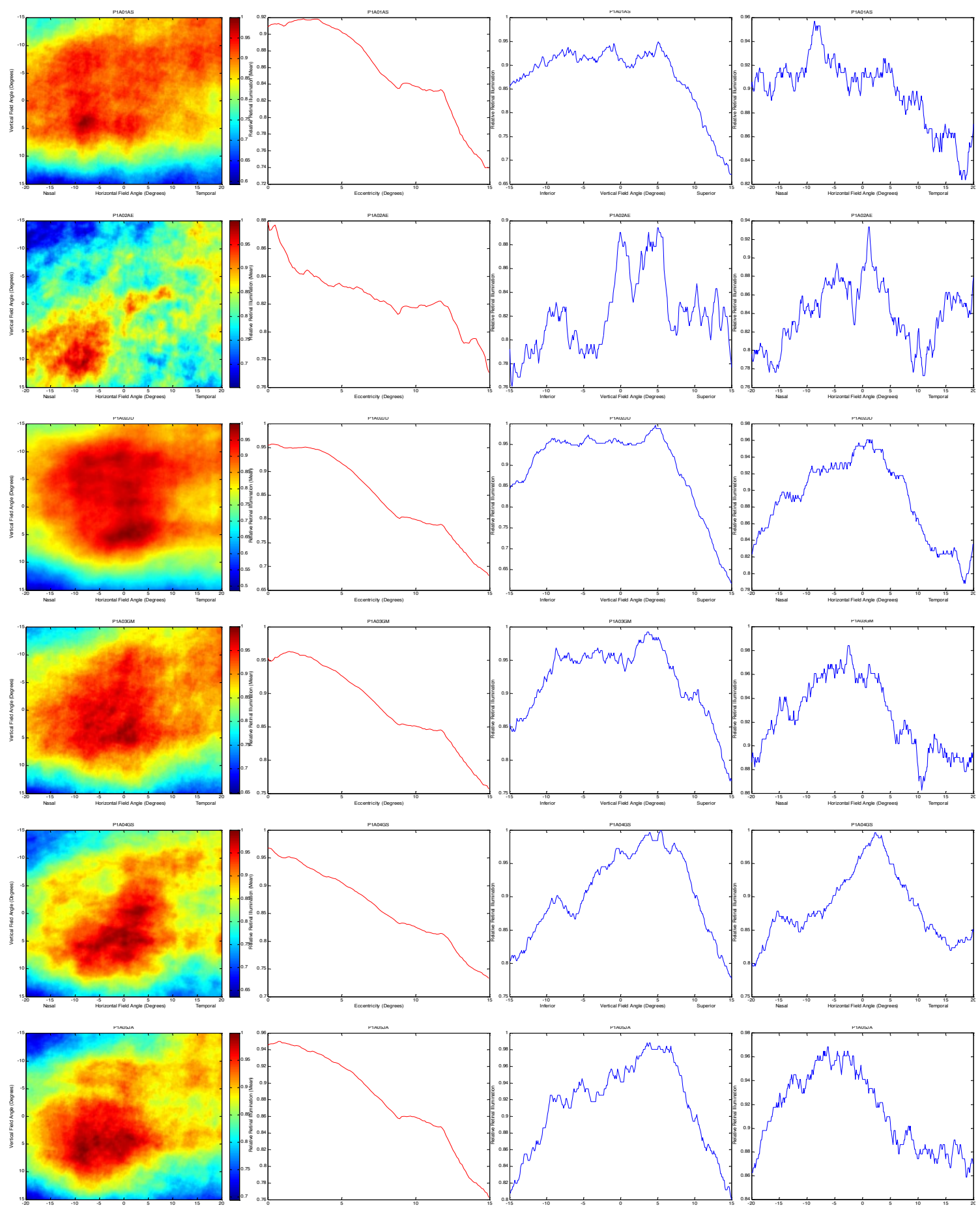



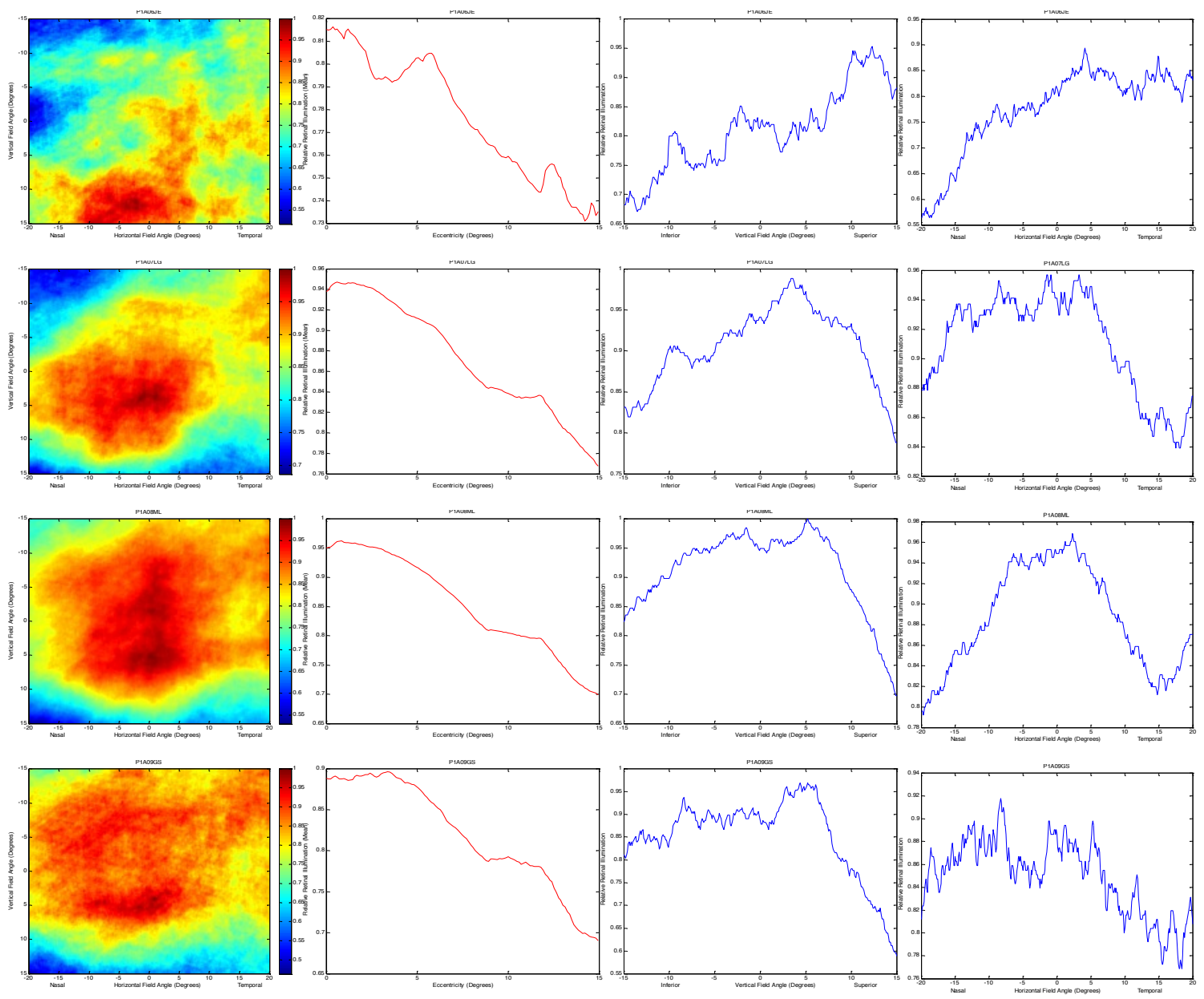


\section{APPENDIX 6}

This appendix contains the individual retinal illumination maps, the horizontal and vertical line-scans across the fovea, and the retinal illumination versus radial eccentricity from the fovea for the subjects between 21 and 35 years old that participated in Phase 1. (P1B)

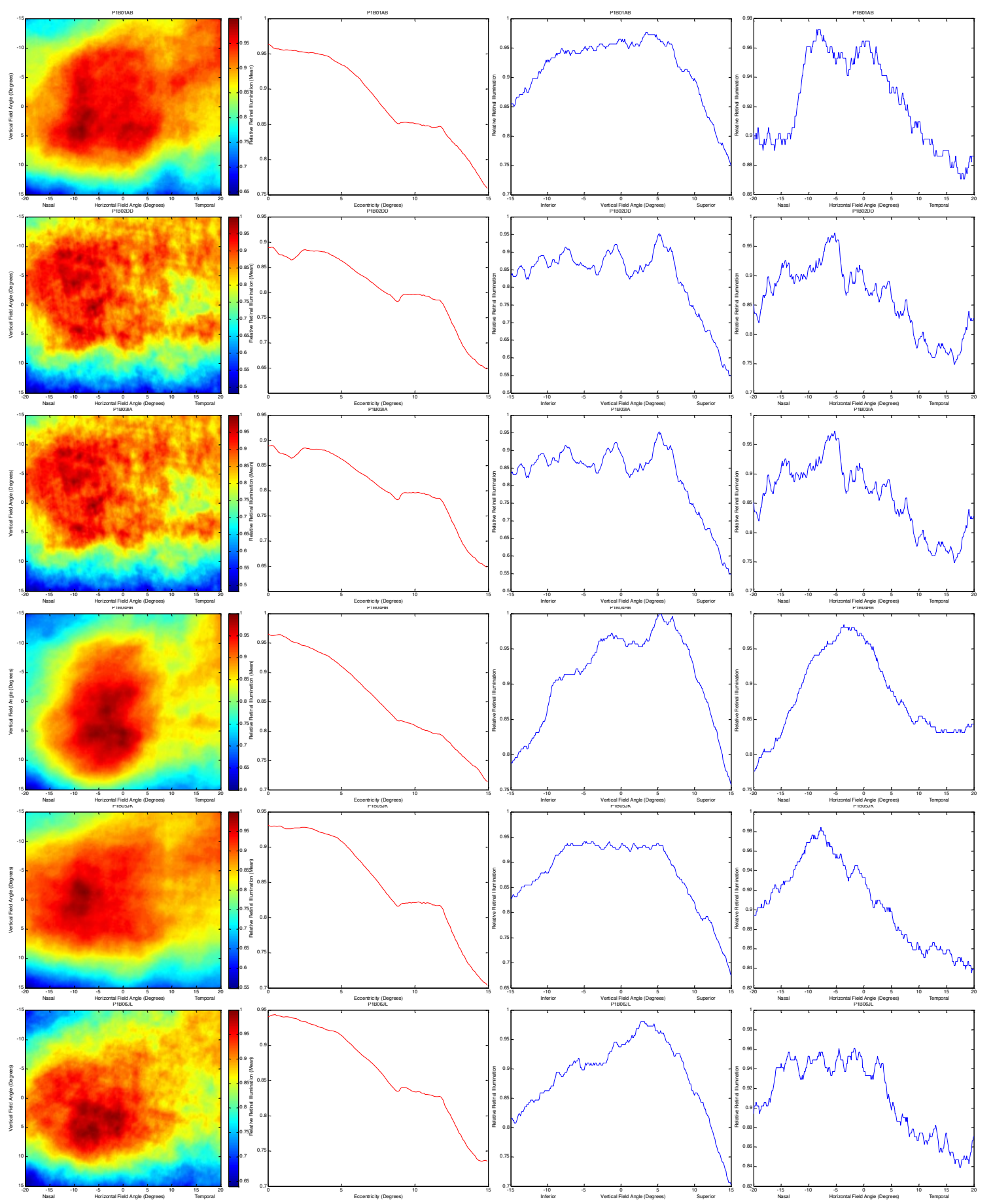



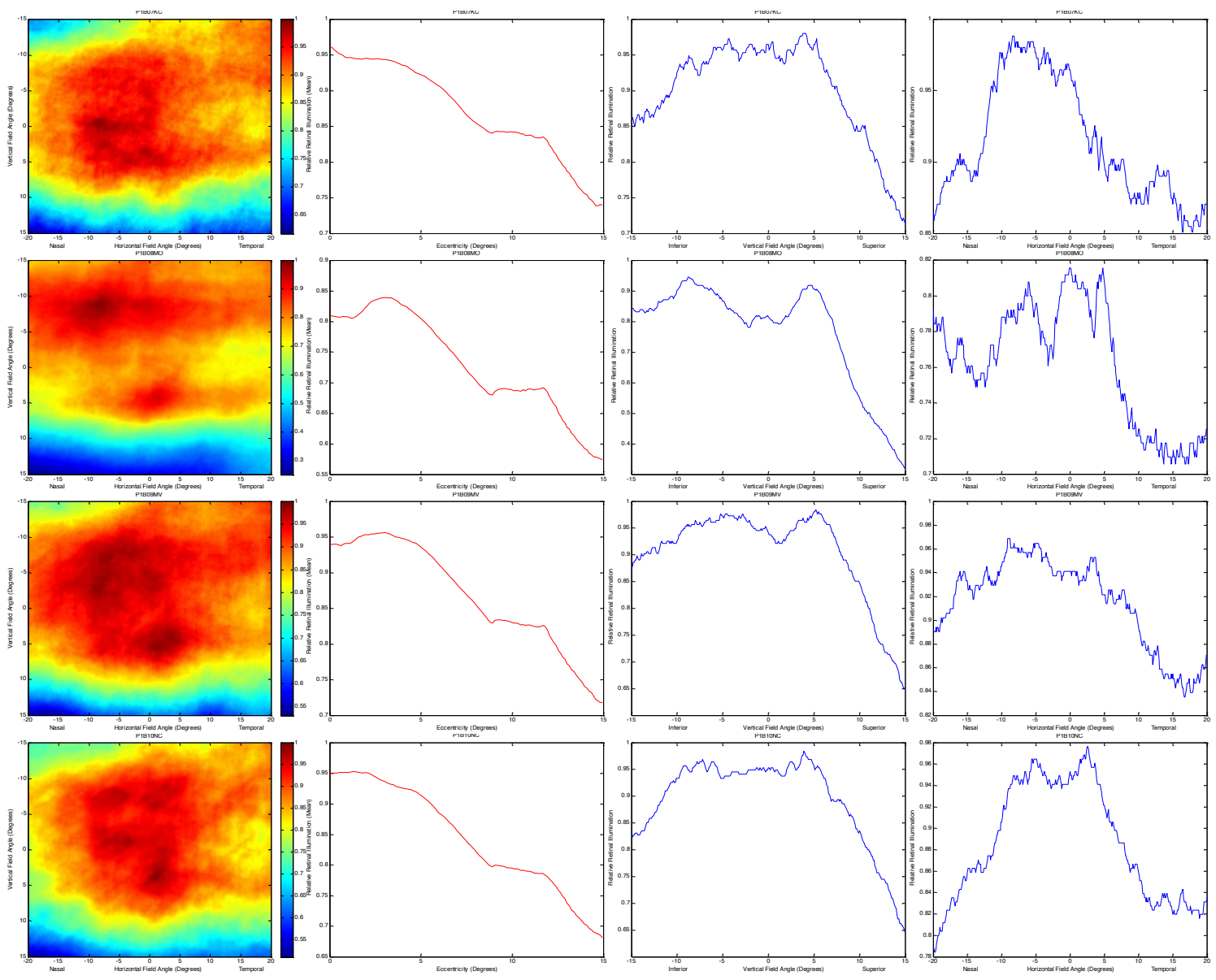


\section{APPENDIX 7}

This appendix contains the individual retinal illumination maps, the horizontal and vertical line-scans across the fovea, and the retinal illumination versus radial eccentricity from the fovea for the subjects over 35 years old that participated in Phase 1. (P1C)
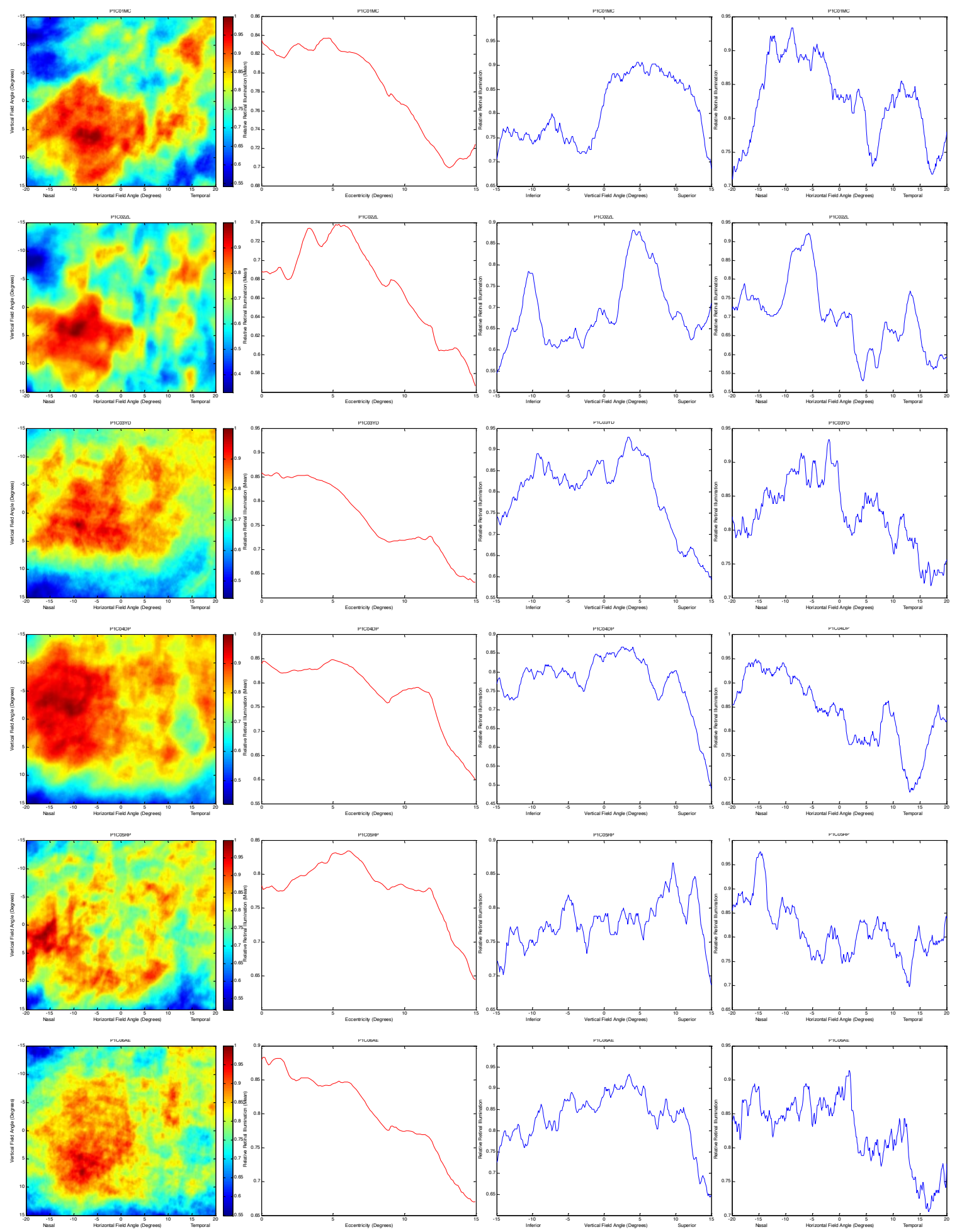

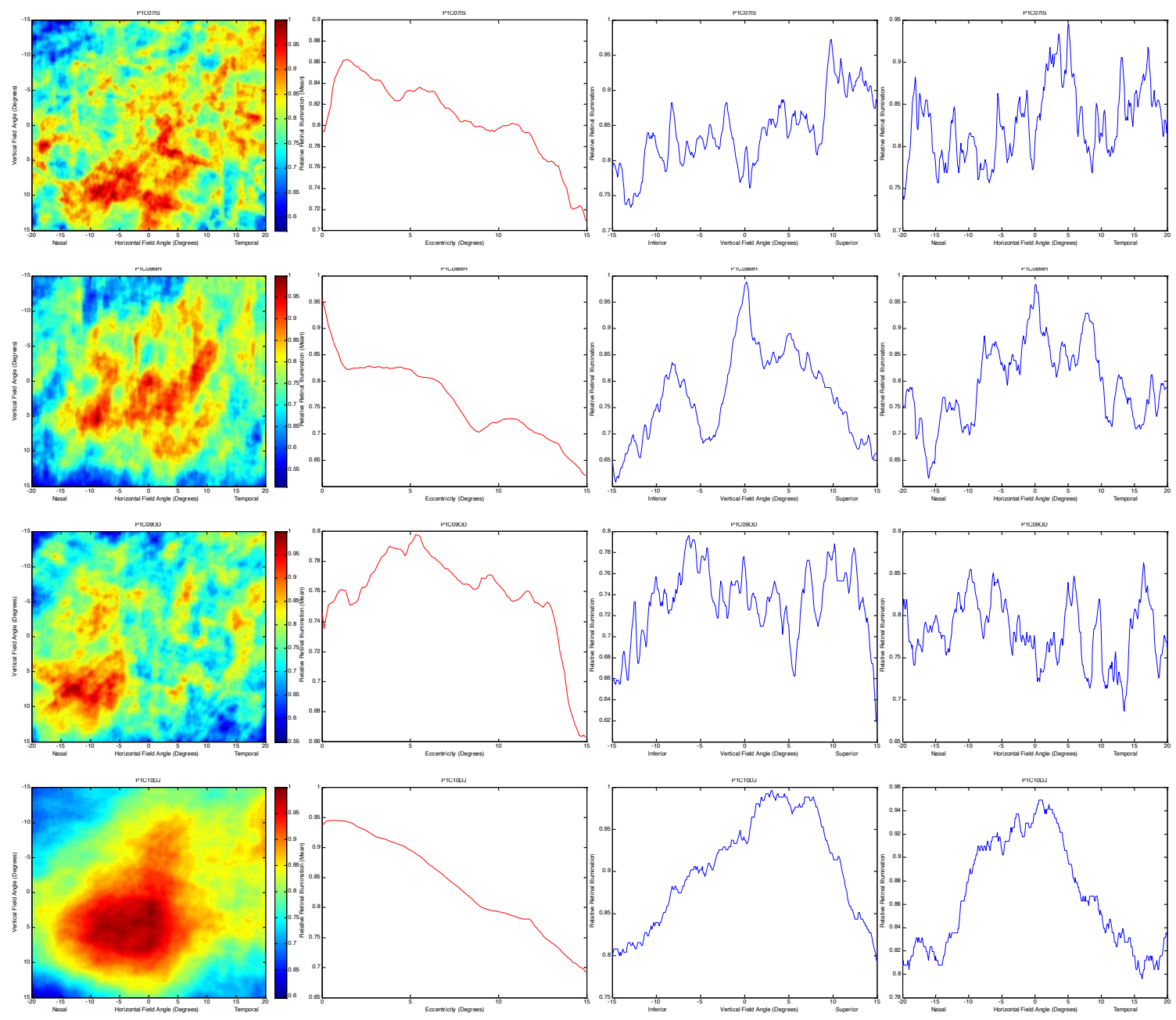


\section{APPENDIX 8}

This appendix contains the individual retinal illumination maps, the horizontal and vertical line-scans across the fovea, and the retinal illumination versus radial eccentricity from the fovea for the subjects under 21 years old that participated in Phase 2. (P2A)
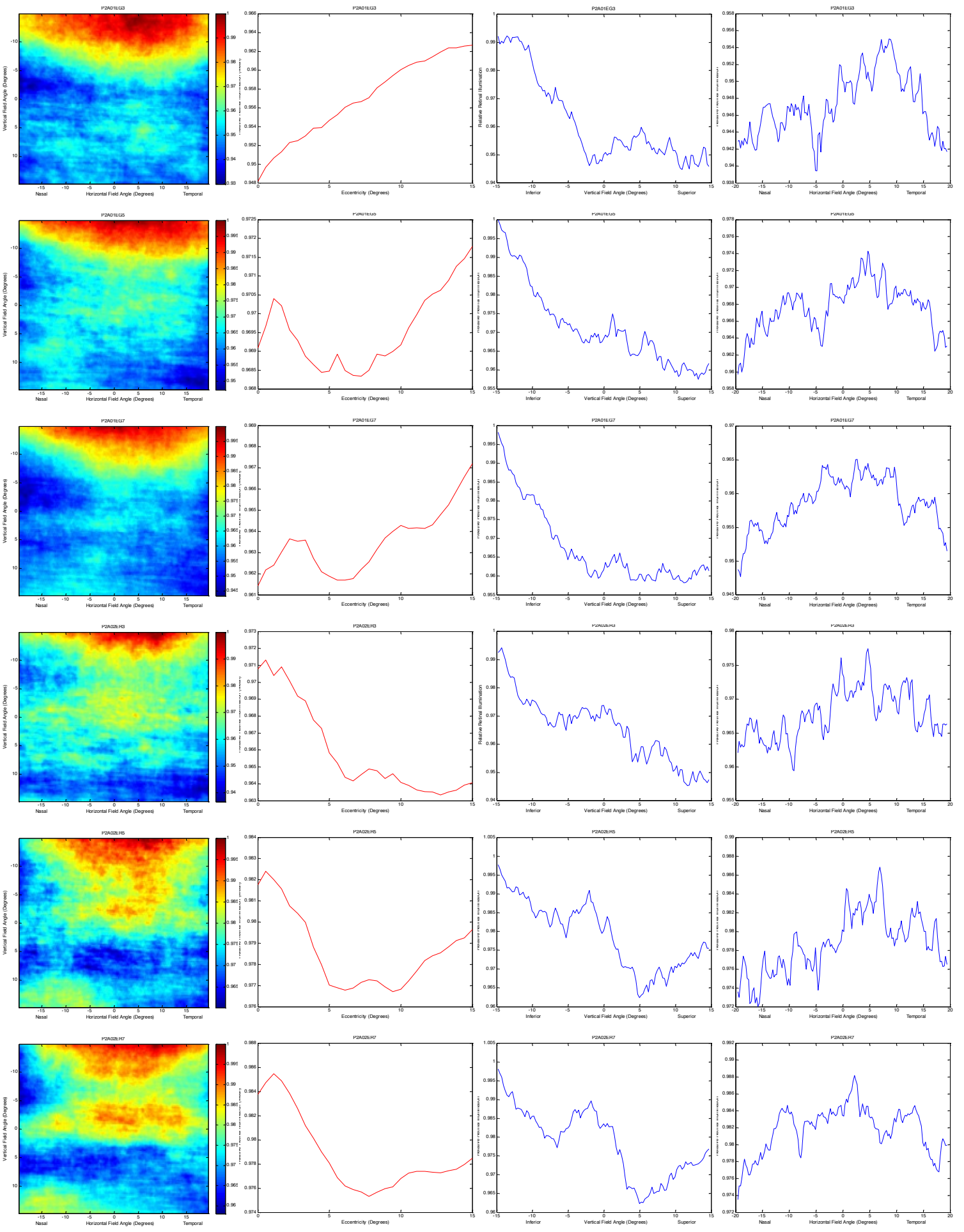

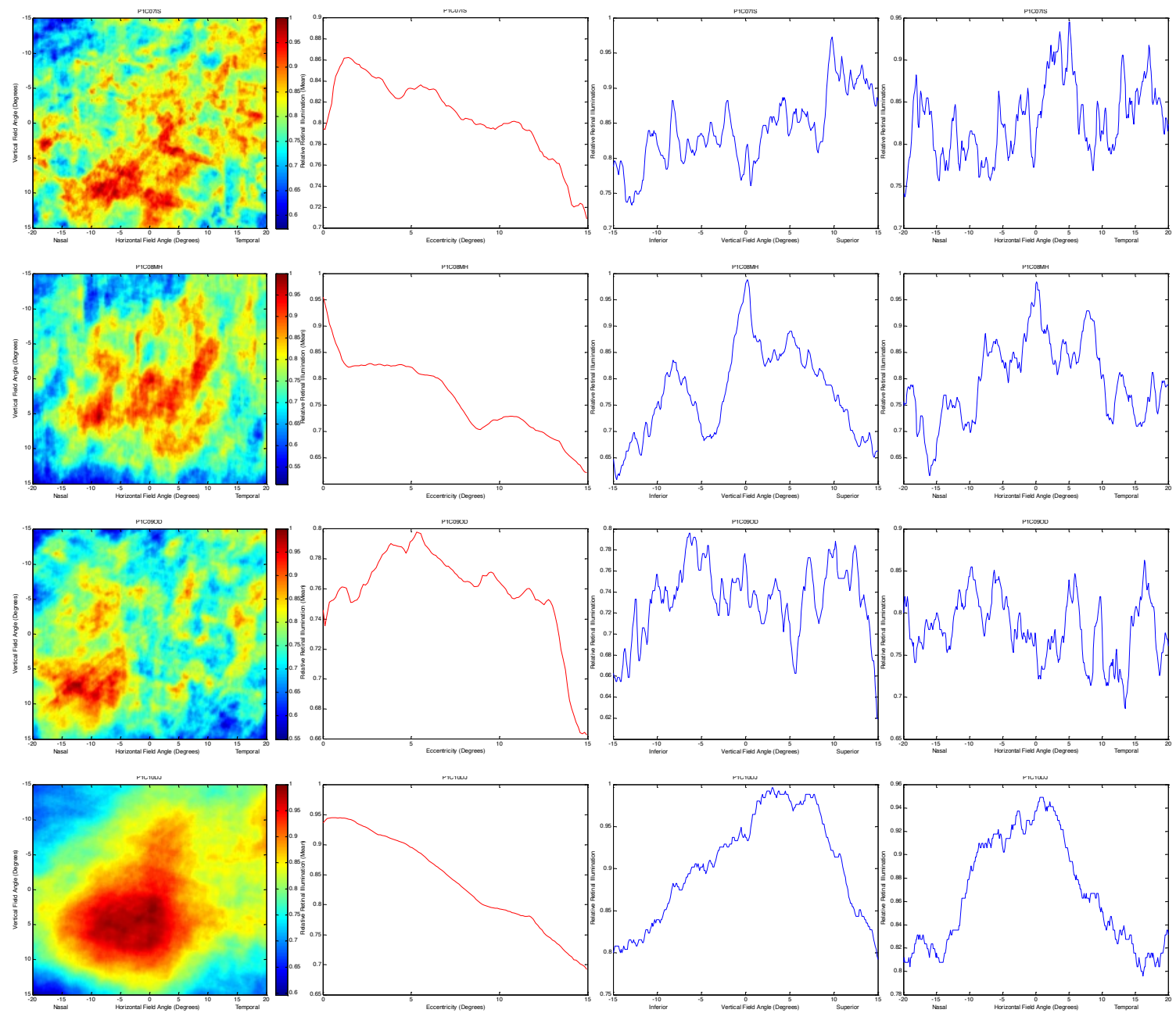


\section{APPENDIX 9}

This appendix contains the individual retinal illumination maps, the horizontal and vertical line-scans across the fovea, and the retinal illumination versus radial eccentricity from the fovea for the subjects between 21 and 35 years old that participated in Phase 2. (P2B)
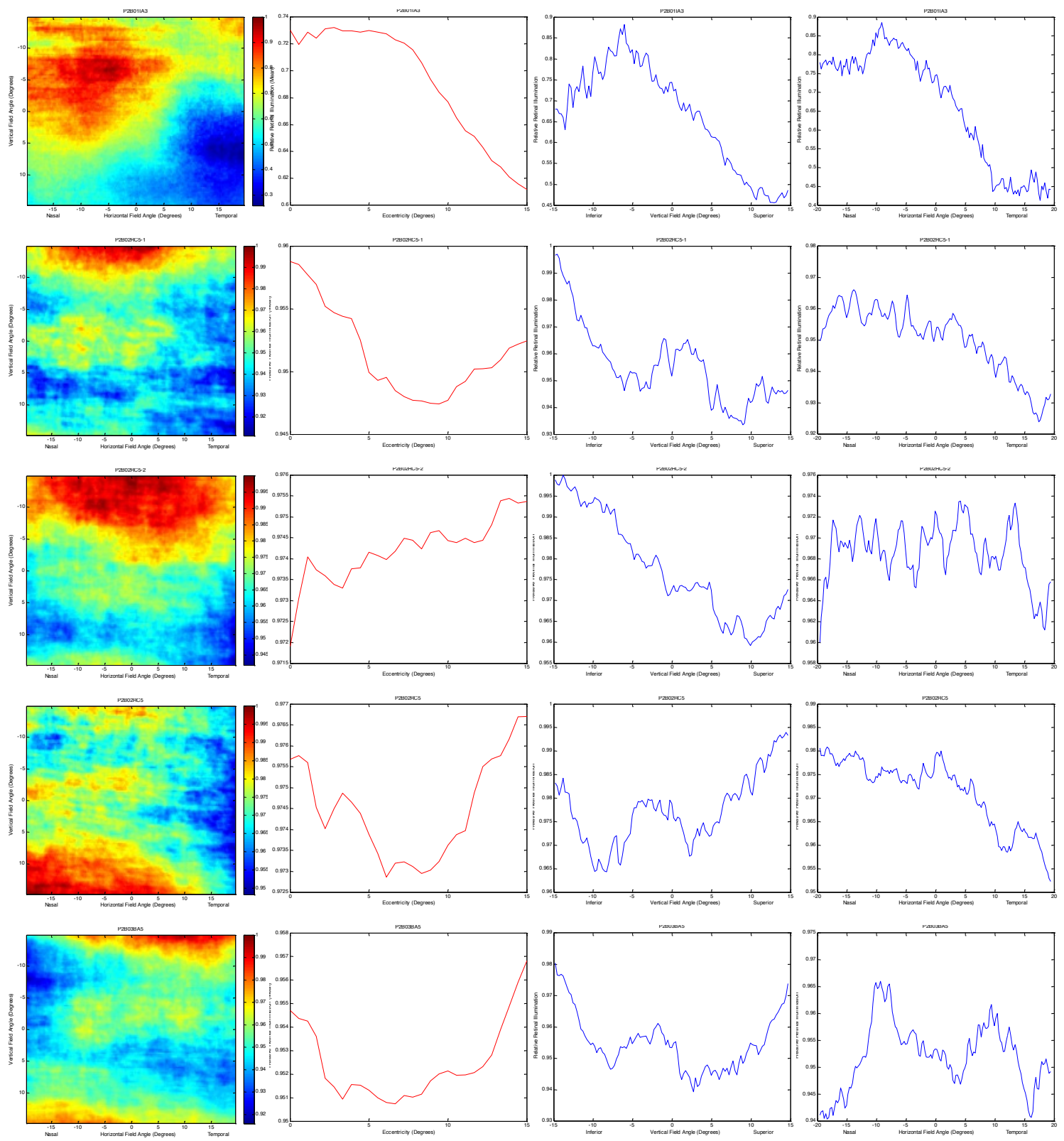


\section{APPENDIX 10}

This appendix contains the individual retinal illumination maps, the horizontal and vertical line-scans across the fovea, and the retinal illumination versus radial eccentricity from the fovea for the subjects over 35 years old that participated in Phase 2. (P2C)
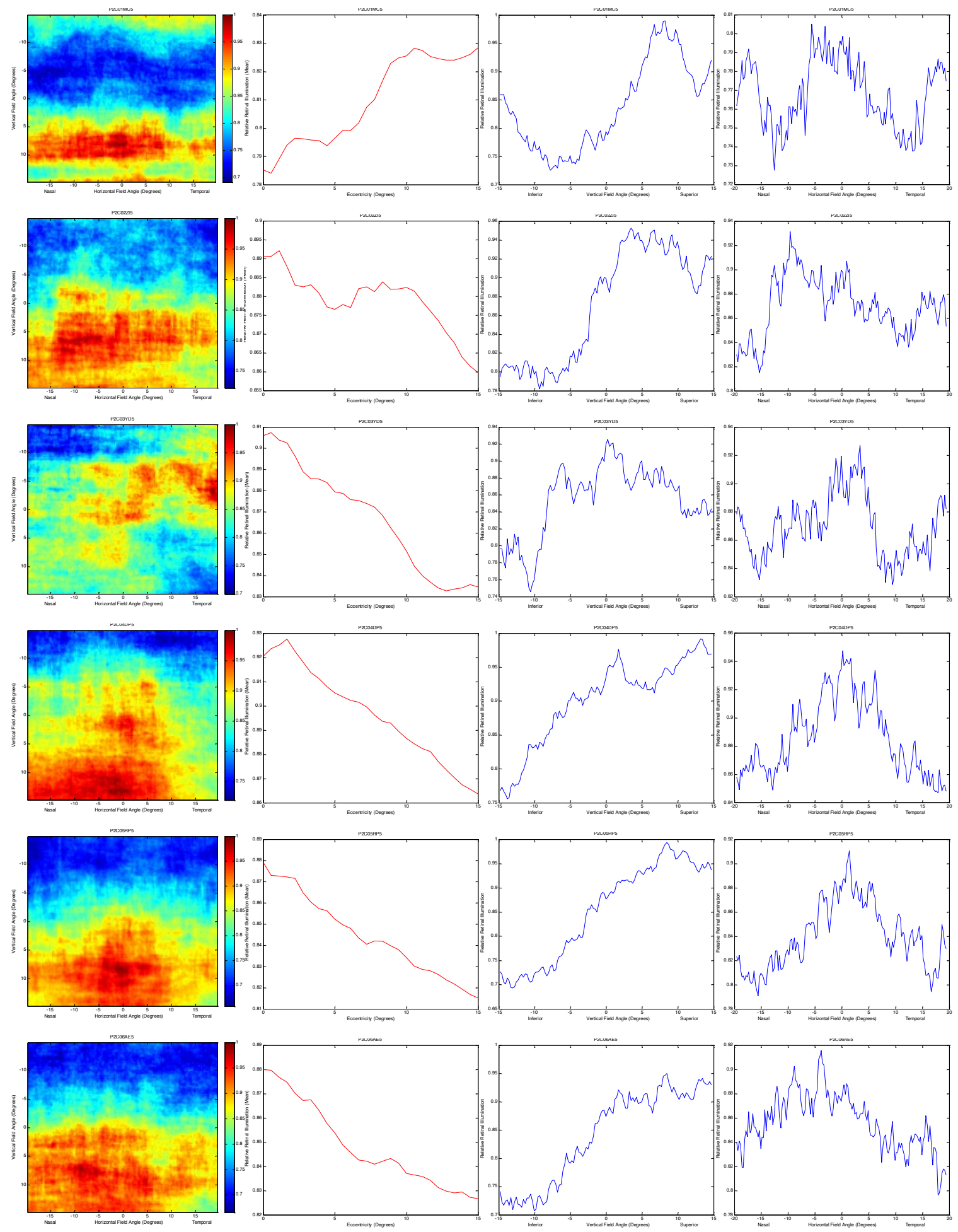

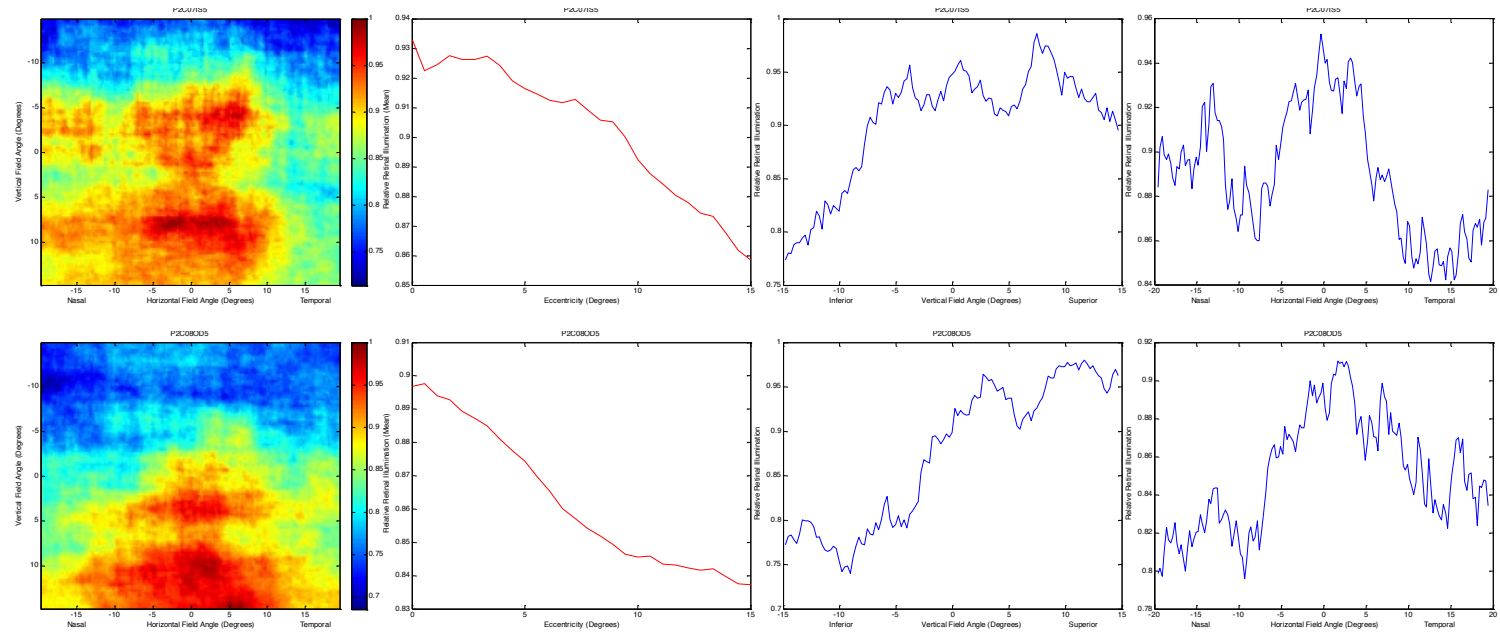


\section{APPENDIX 11}

This appendix contains the individual retinal illumination maps, the horizontal and vertical line-scans across the fovea, and the retinal illumination versus radial eccentricity from the fovea for the subjects under 21 years old that participated in Phase 3. (P3A)
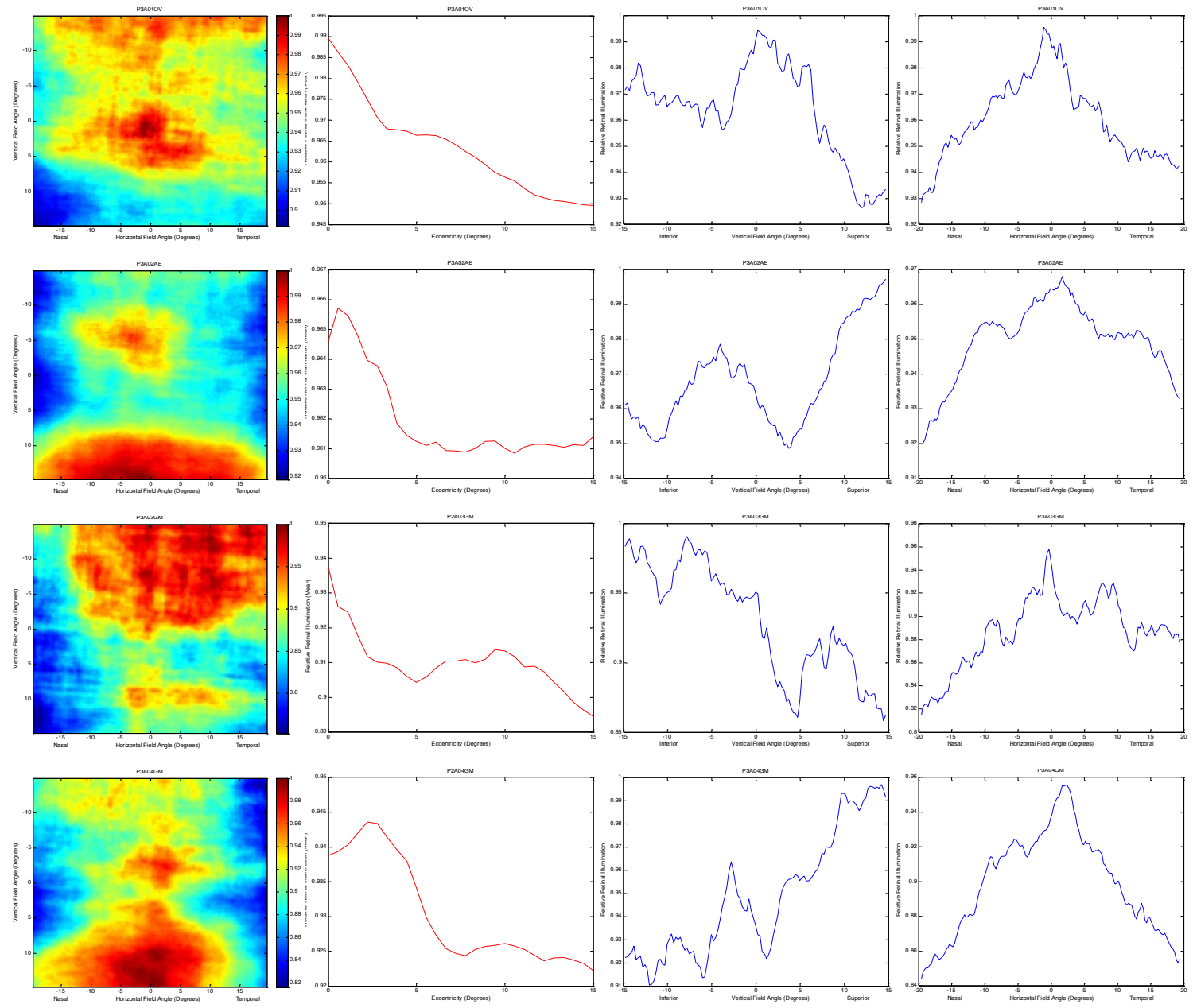


\section{APPENDIX 12}

This appendix contains the individual retinal illumination maps, the horizontal and vertical line-scans across the fovea, and the retinal illumination versus radial eccentricity from the fovea for the subjects between 21 and 35 years old that participated in Phase 3. (P3B)
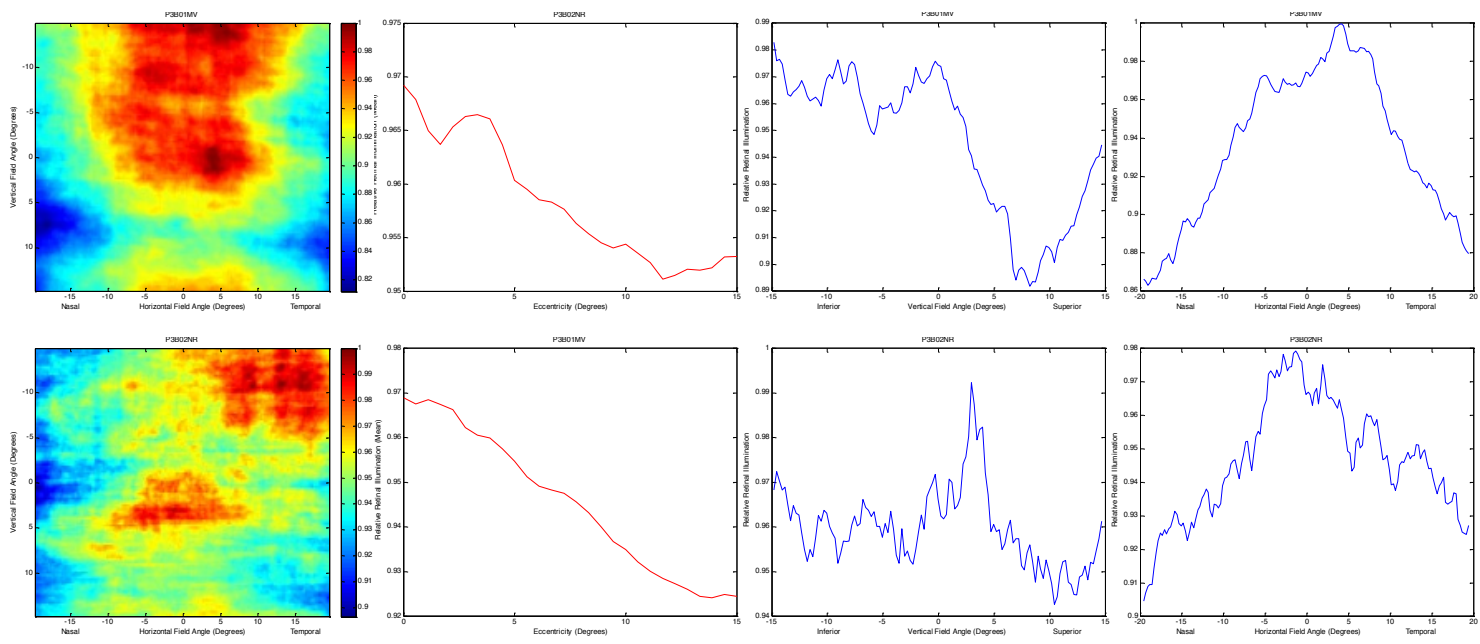


\section{APPENDIX 13}

This appendix contains the individual retinal illumination maps, the horizontal and vertical line-scans across the fovea, and the retinal illumination versus radial eccentricity from the fovea for the subjects over 35 years old that participated in Phase 3. (P3C)
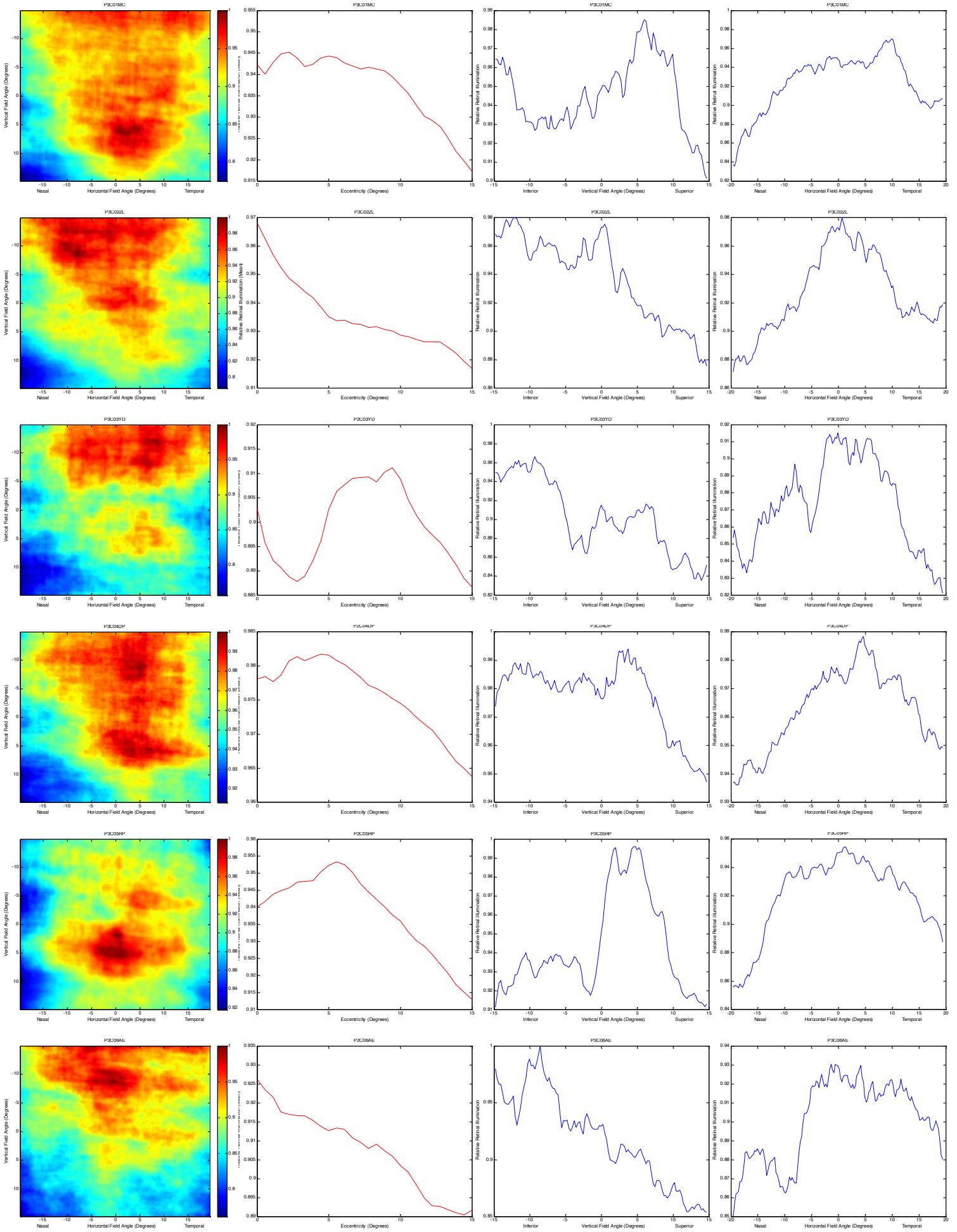

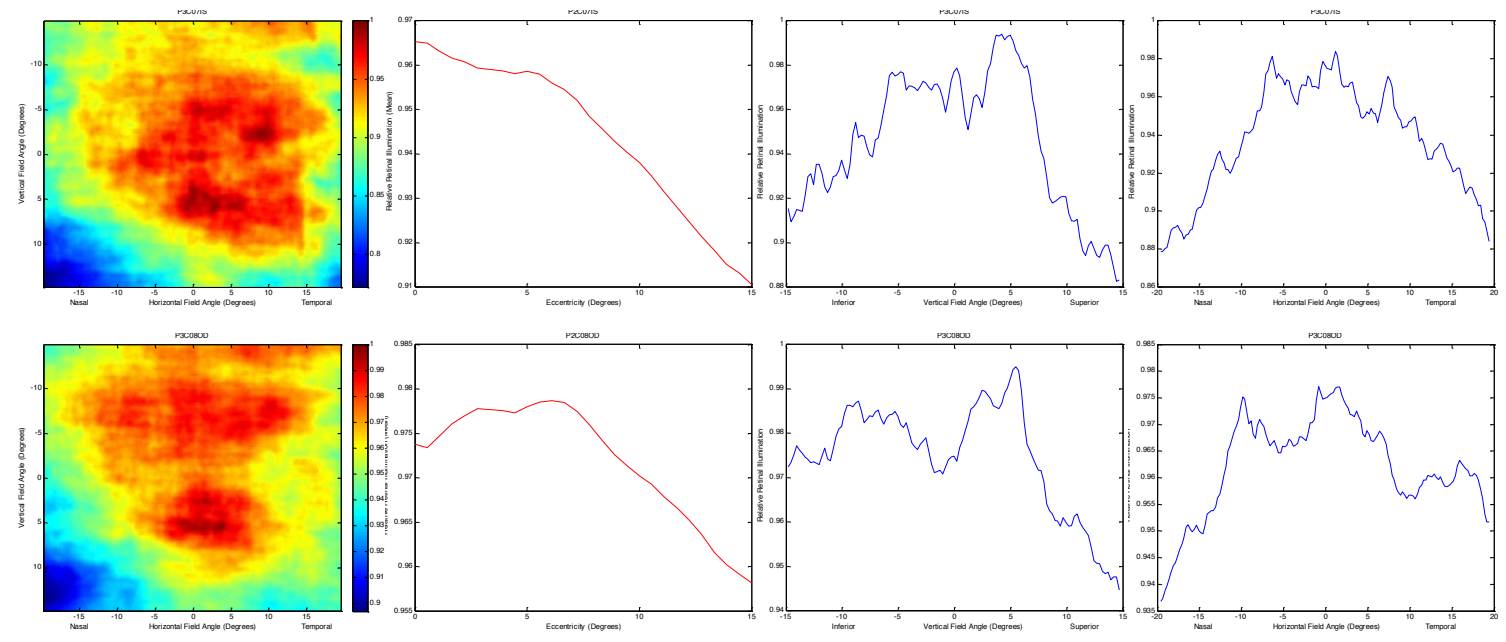


\section{APPENDIX 14}

This appendix contains the individual retinal illumination maps, the horizontal and vertical line-scans across the fovea, and the retinal illumination versus radial eccentricity from the fovea for the subjects under 21 years old that participated in Phase 4. (P4A)
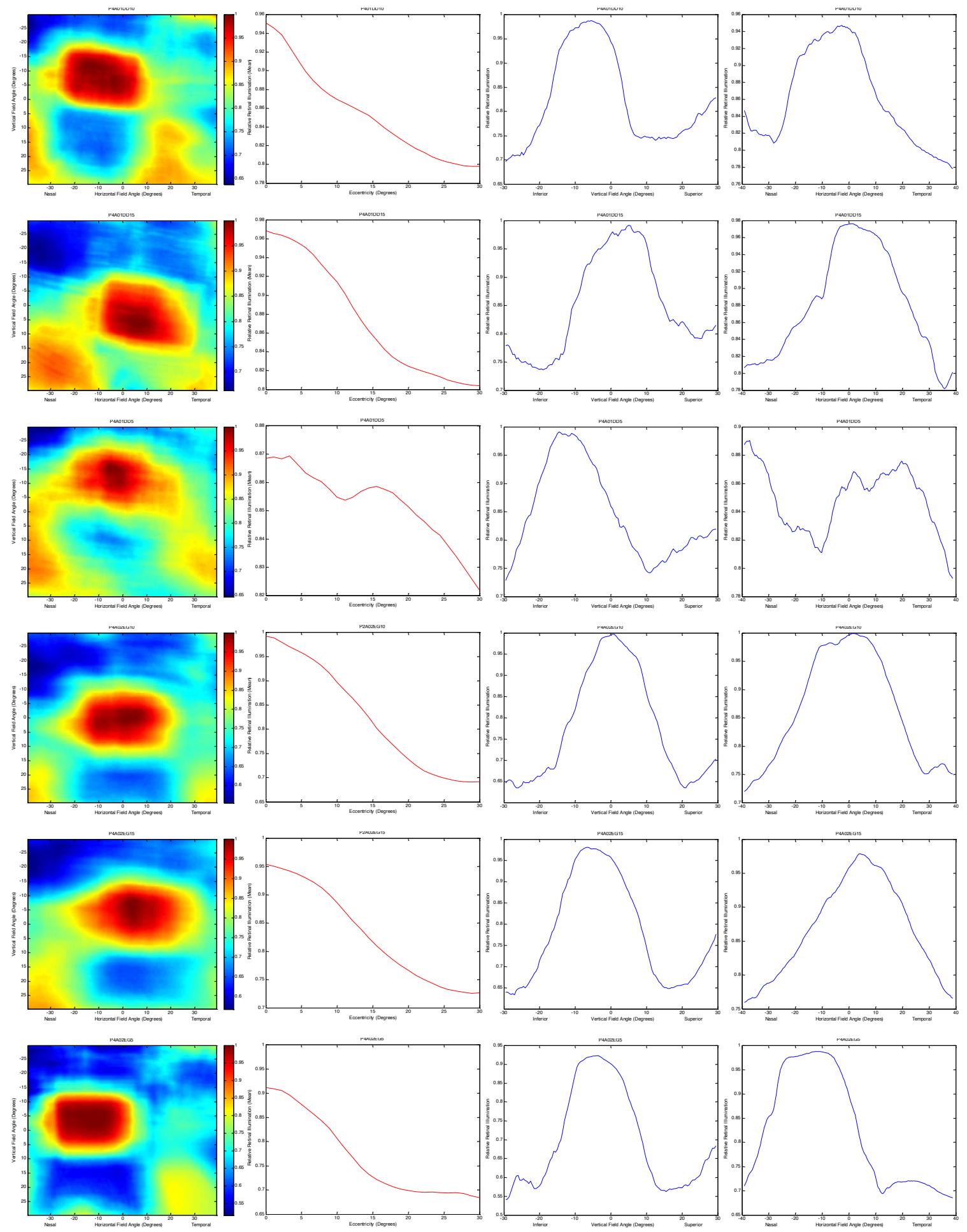

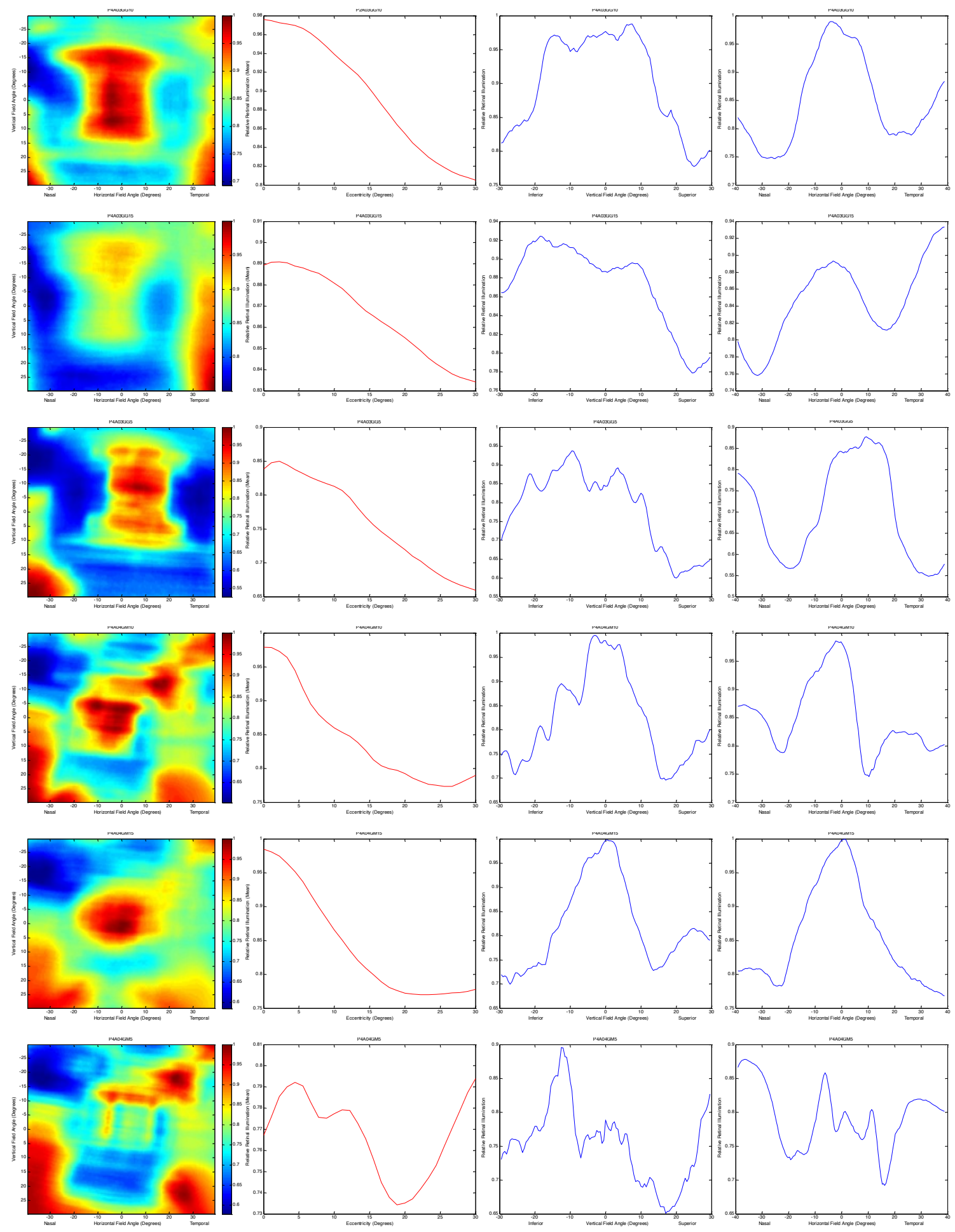

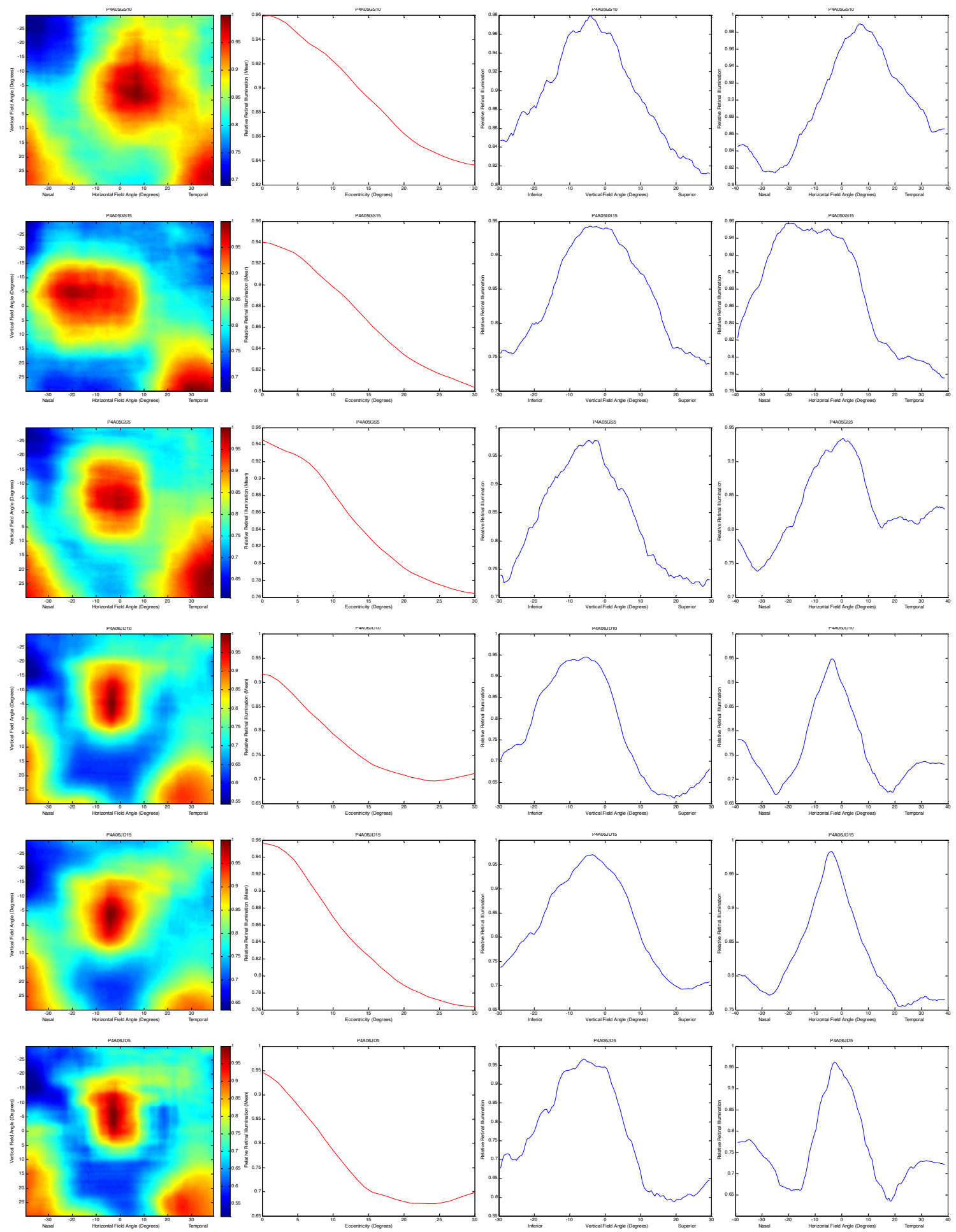

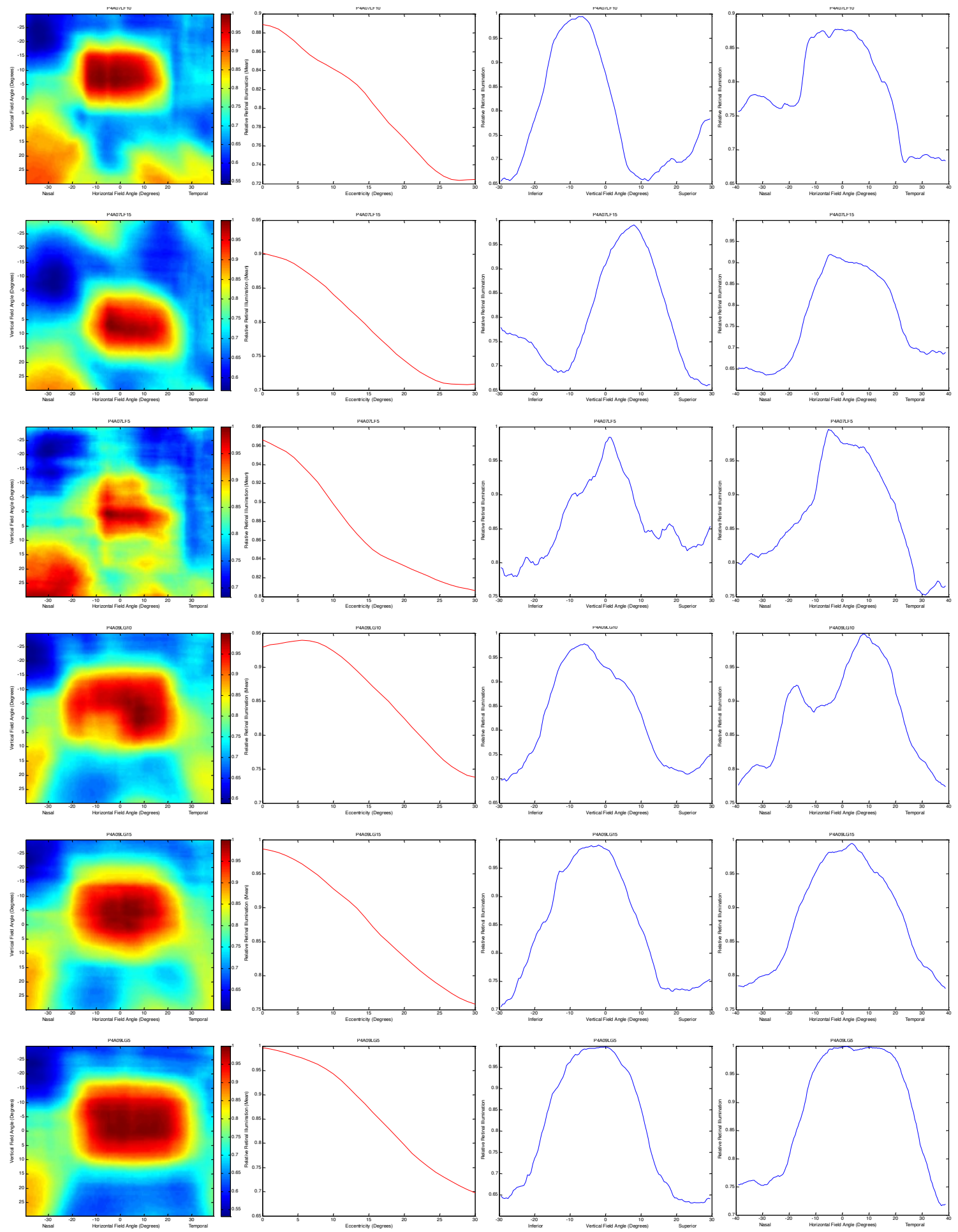

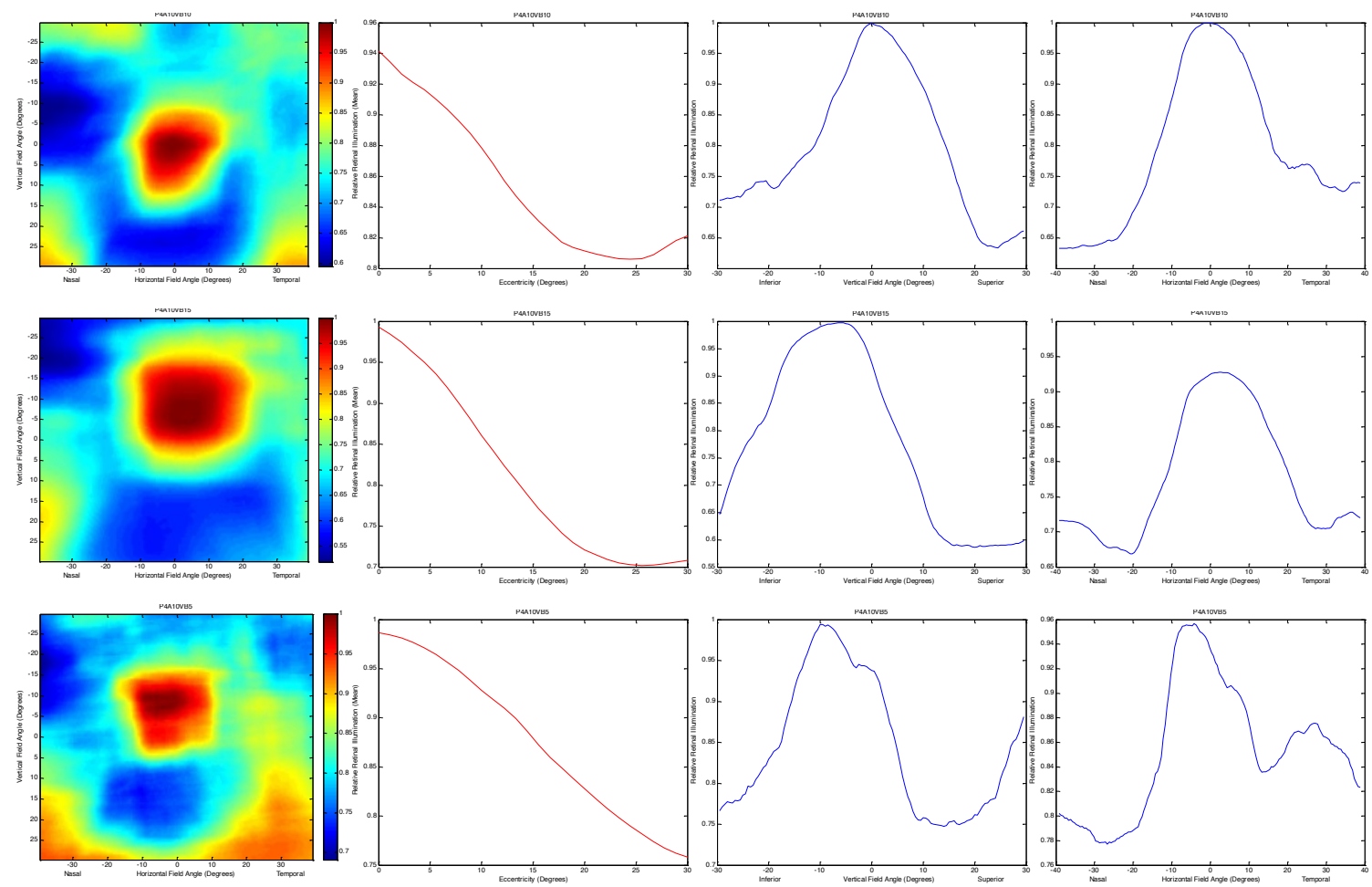


\section{APPENDIX 15}

This appendix contains the individual retinal illumination maps, the horizontal and vertical line-scans across the fovea, and the retinal illumination versus radial eccentricity from the fovea for the subjects between 21 and 35 years old that participated in Phase 4. (P4B)
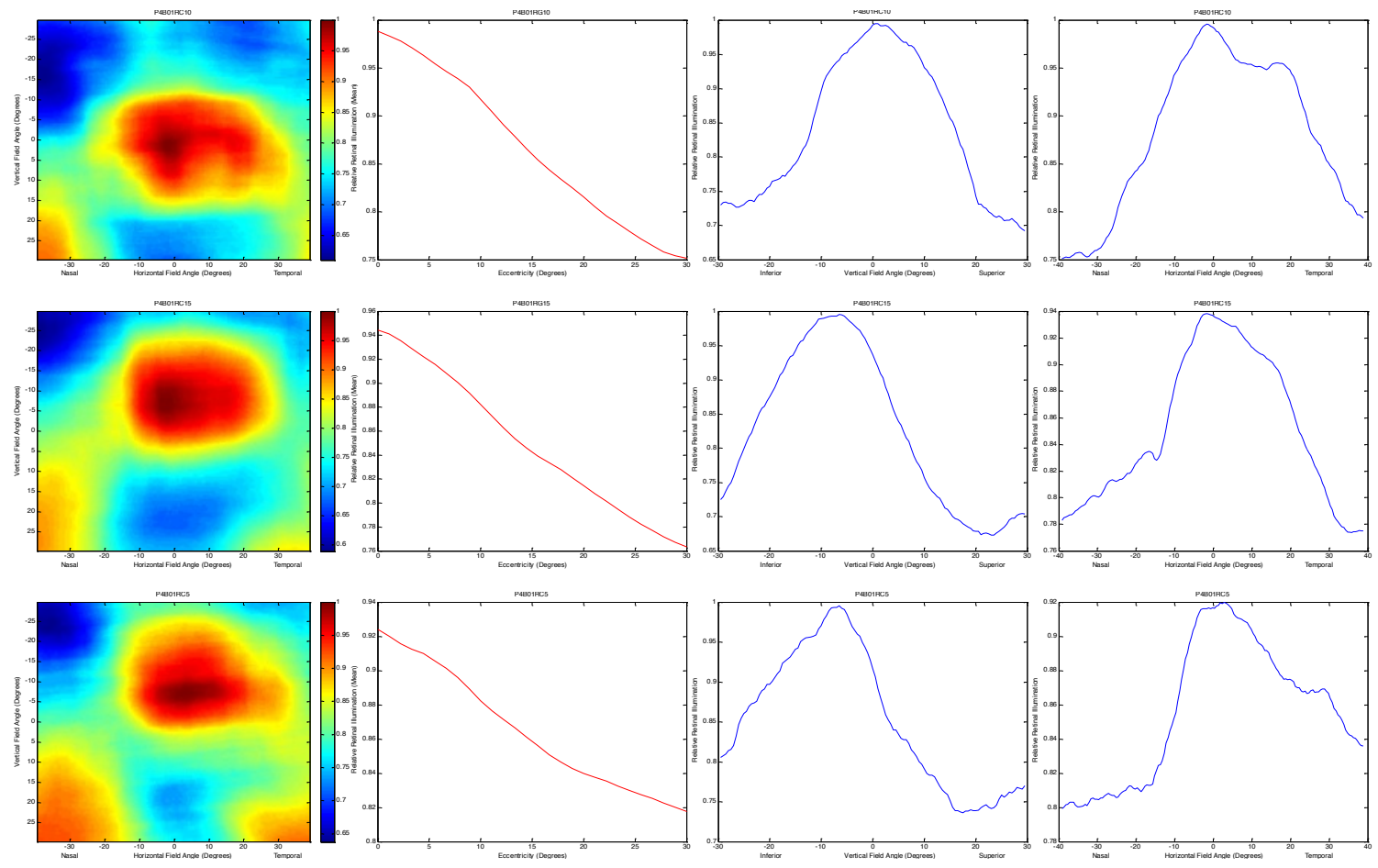


\section{APPENDIX 16}

This appendix contains the individual retinal illumination maps, the horizontal and vertical line-scans across the fovea, and the retinal illumination versus radial eccentricity from the fovea for the subjects under 21 years old that walked freely around the lab and hallways in Phase 5. (P5LA)
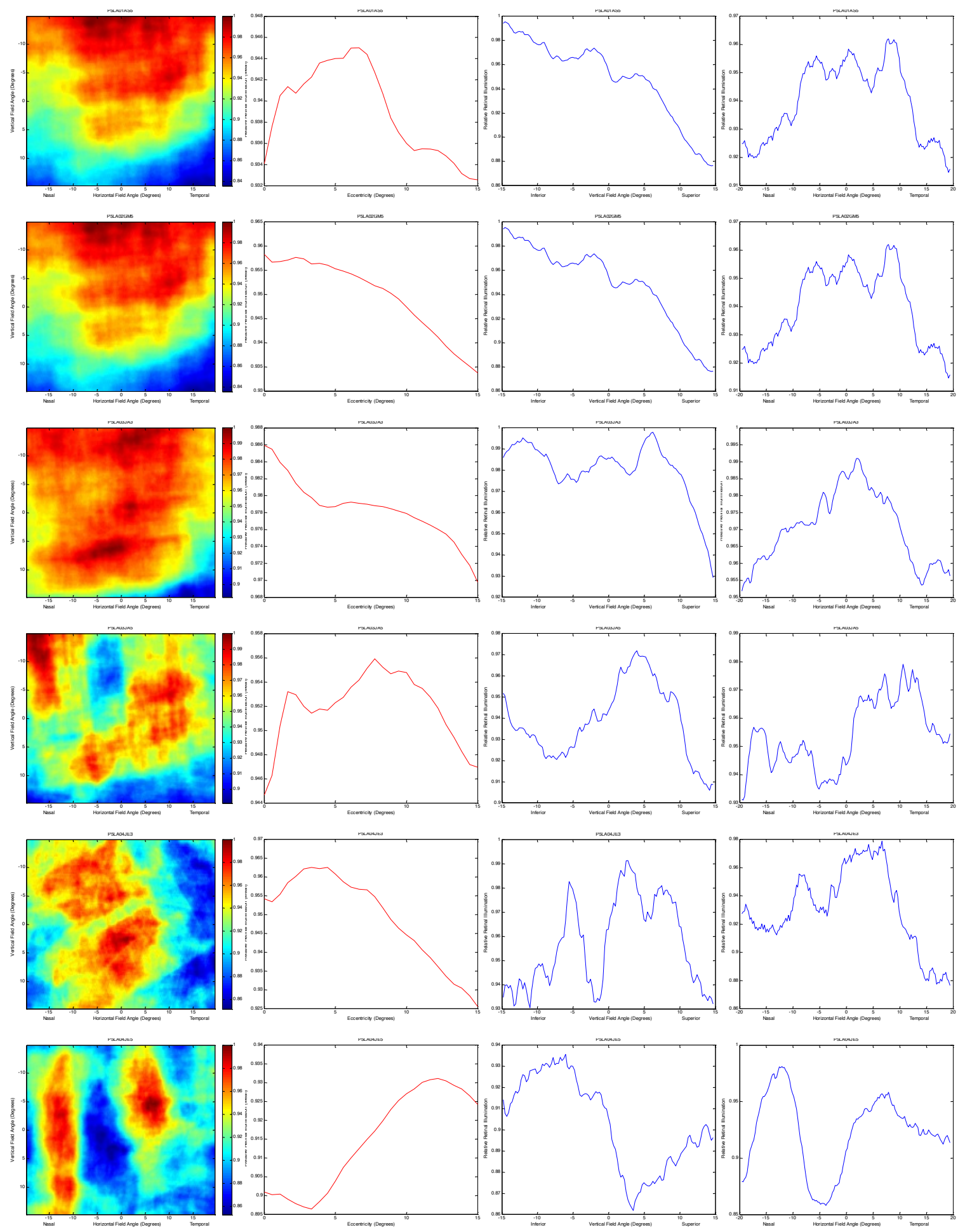

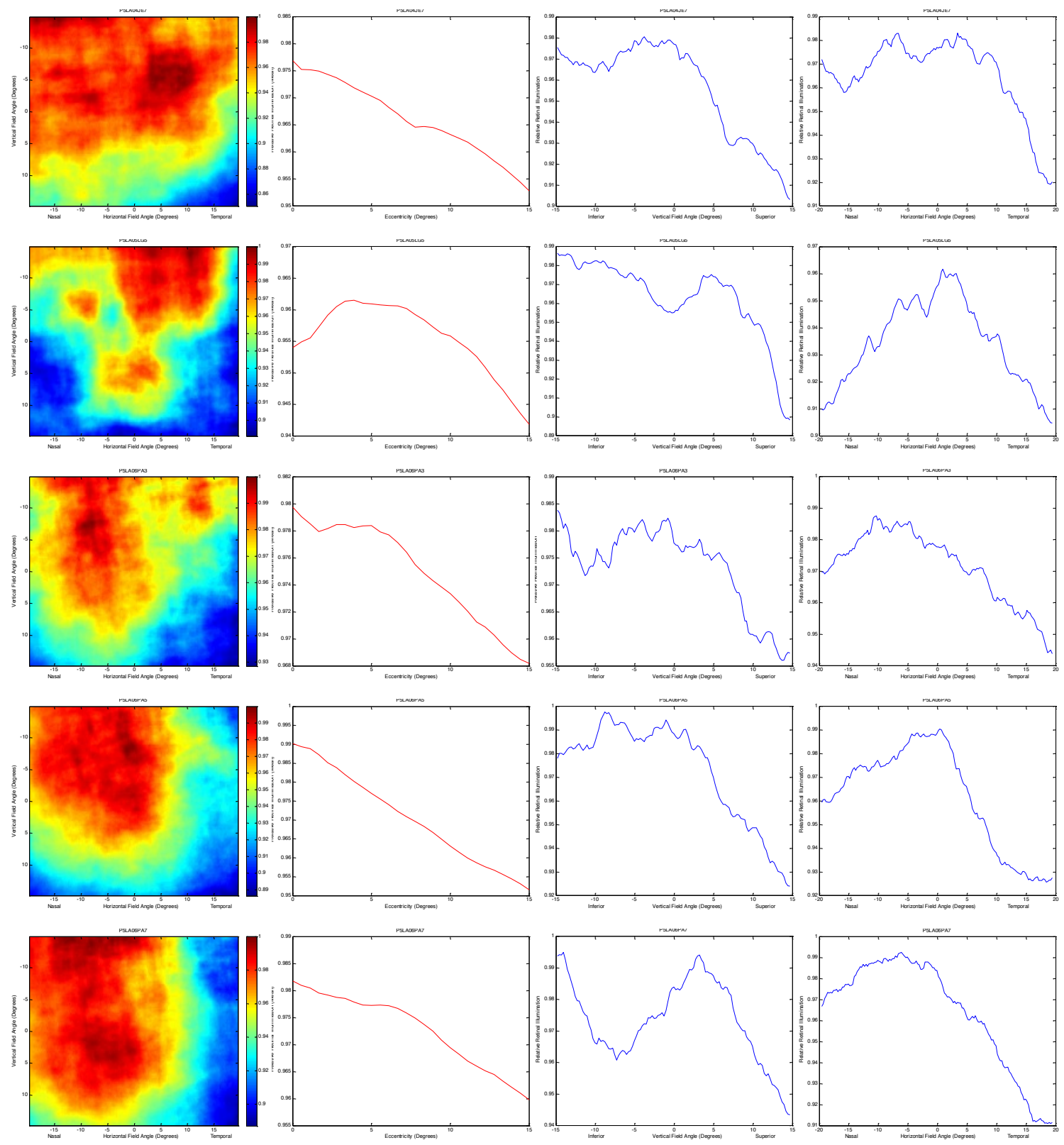


\section{APPENDIX 17}

This appendix contains the individual retinal illumination maps, the horizontal and vertical line-scans across the fovea, and the retinal illumination versus radial eccentricity from the fovea for the subjects between 21 and 35 years old that walked freely around the lab and hallways in Phase 5. (P5LB)
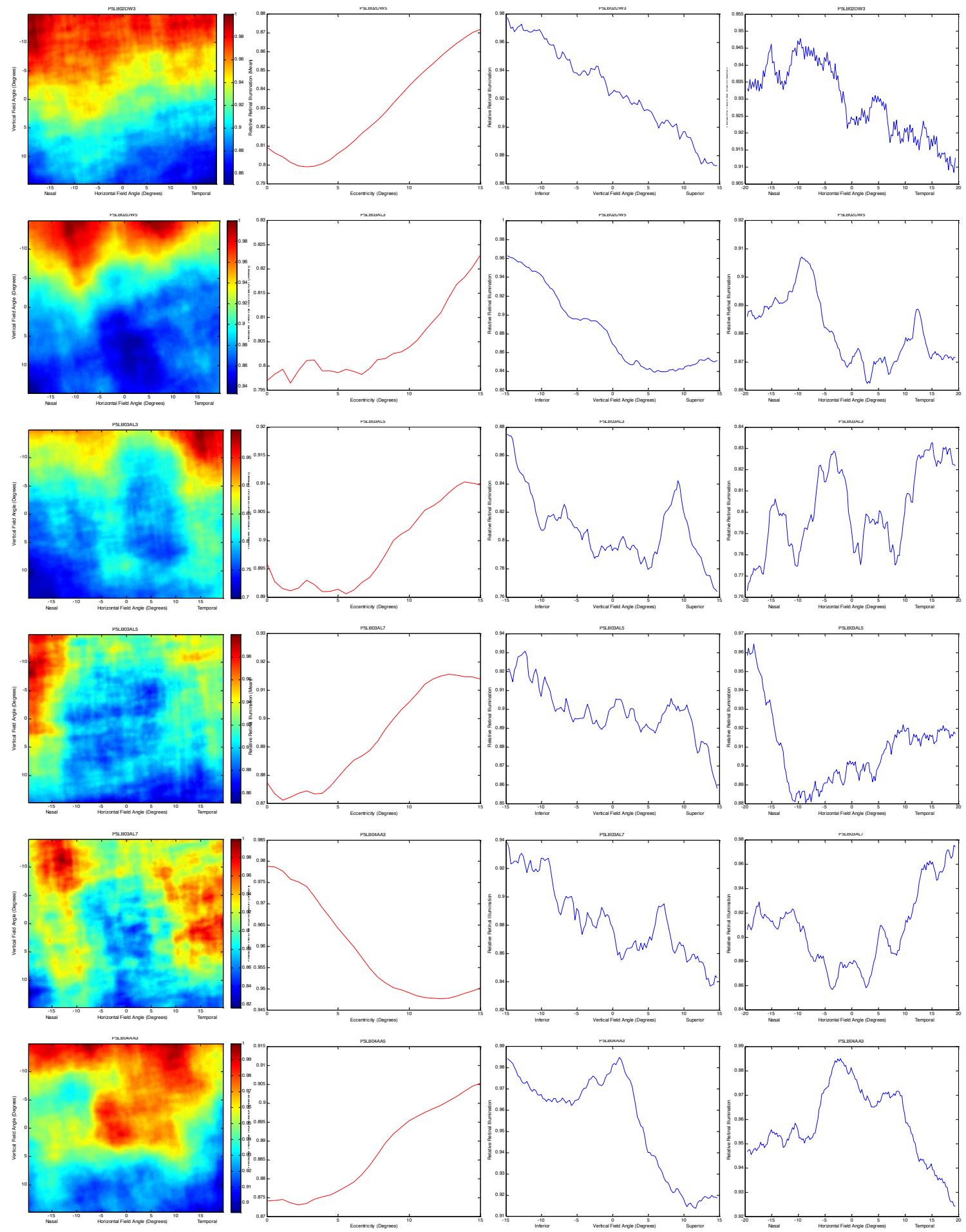

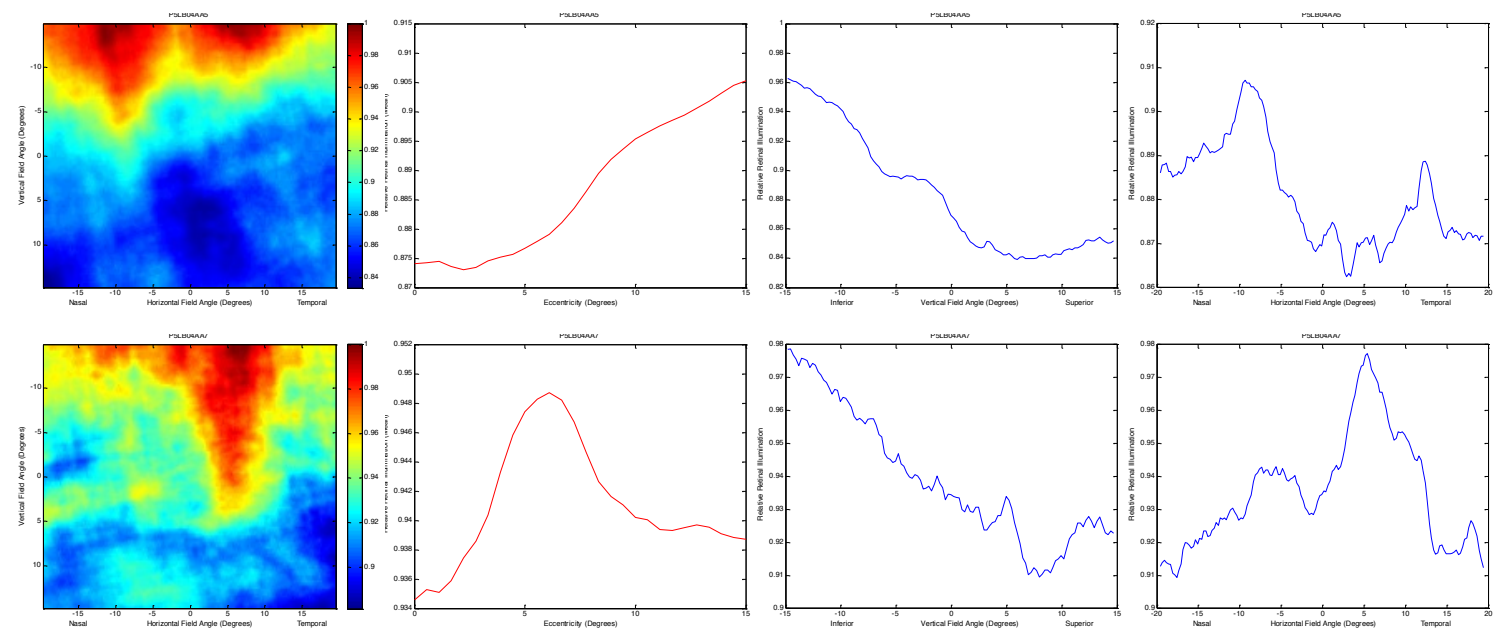


\section{APPENDIX 18}

This appendix contains the individual retinal illumination maps, the horizontal and vertical line-scans across the fovea, and the retinal illumination versus radial eccentricity from the fovea for the subjects over 35 years old that walked freely around the lab and hallways in Phase 5. (P5LC)
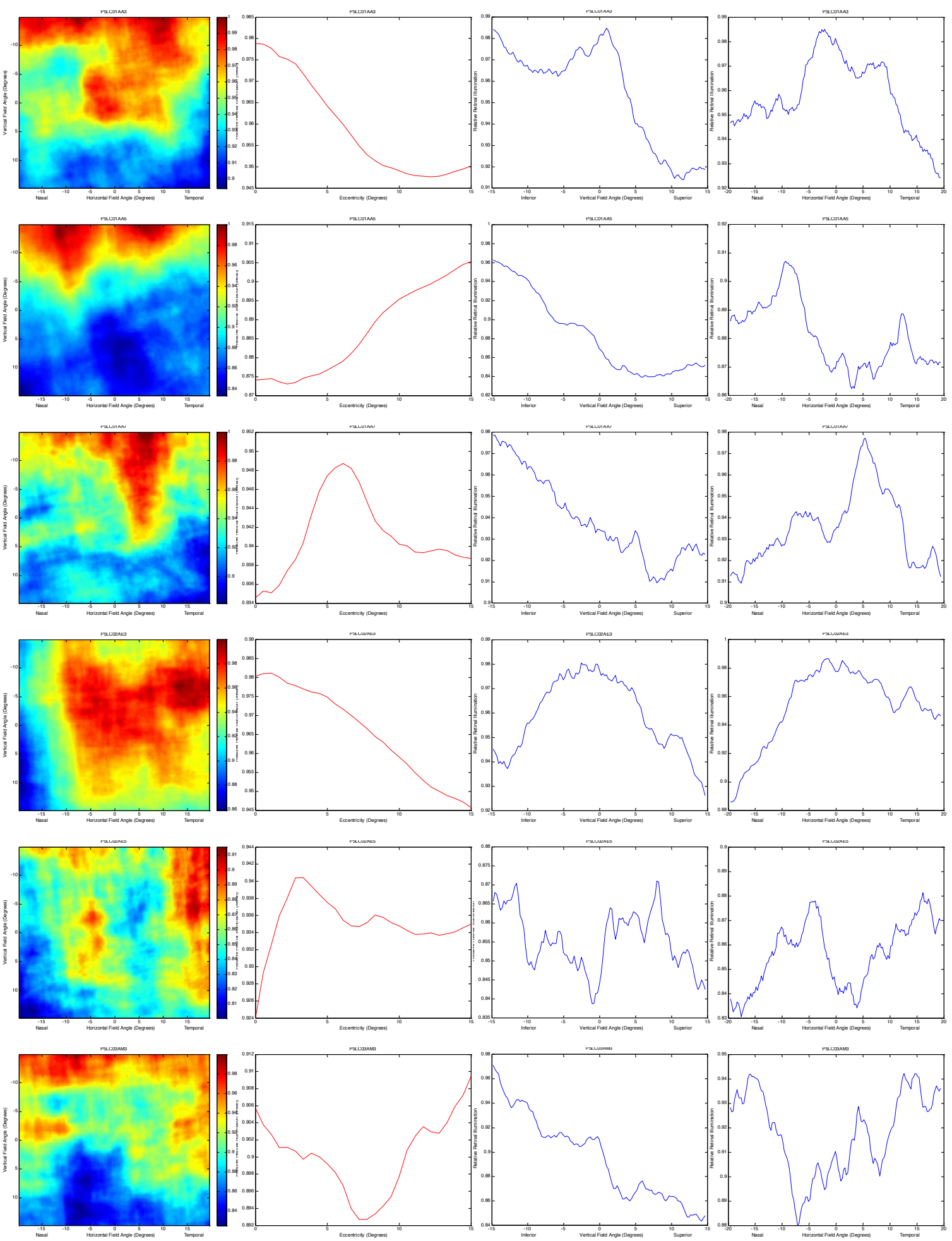

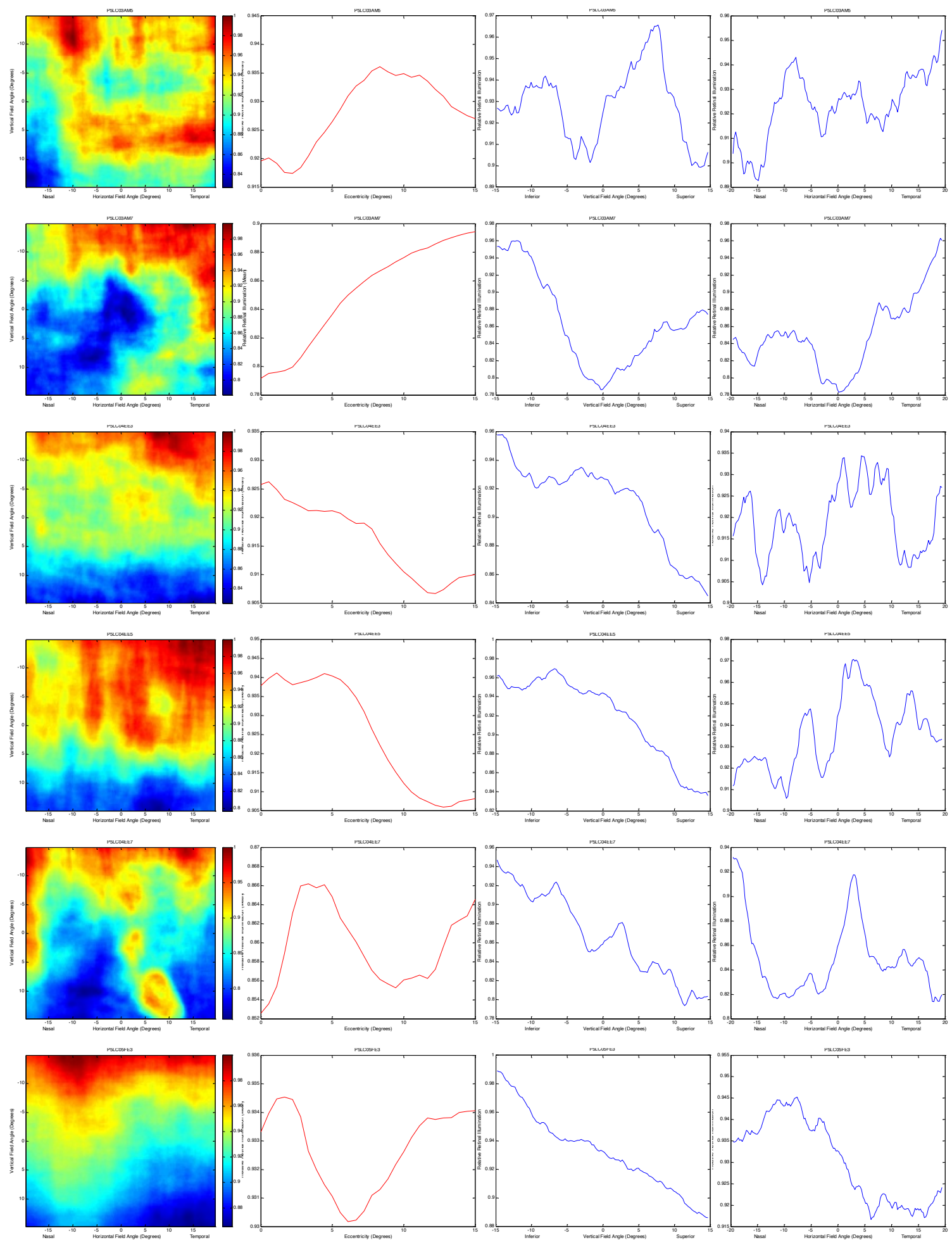

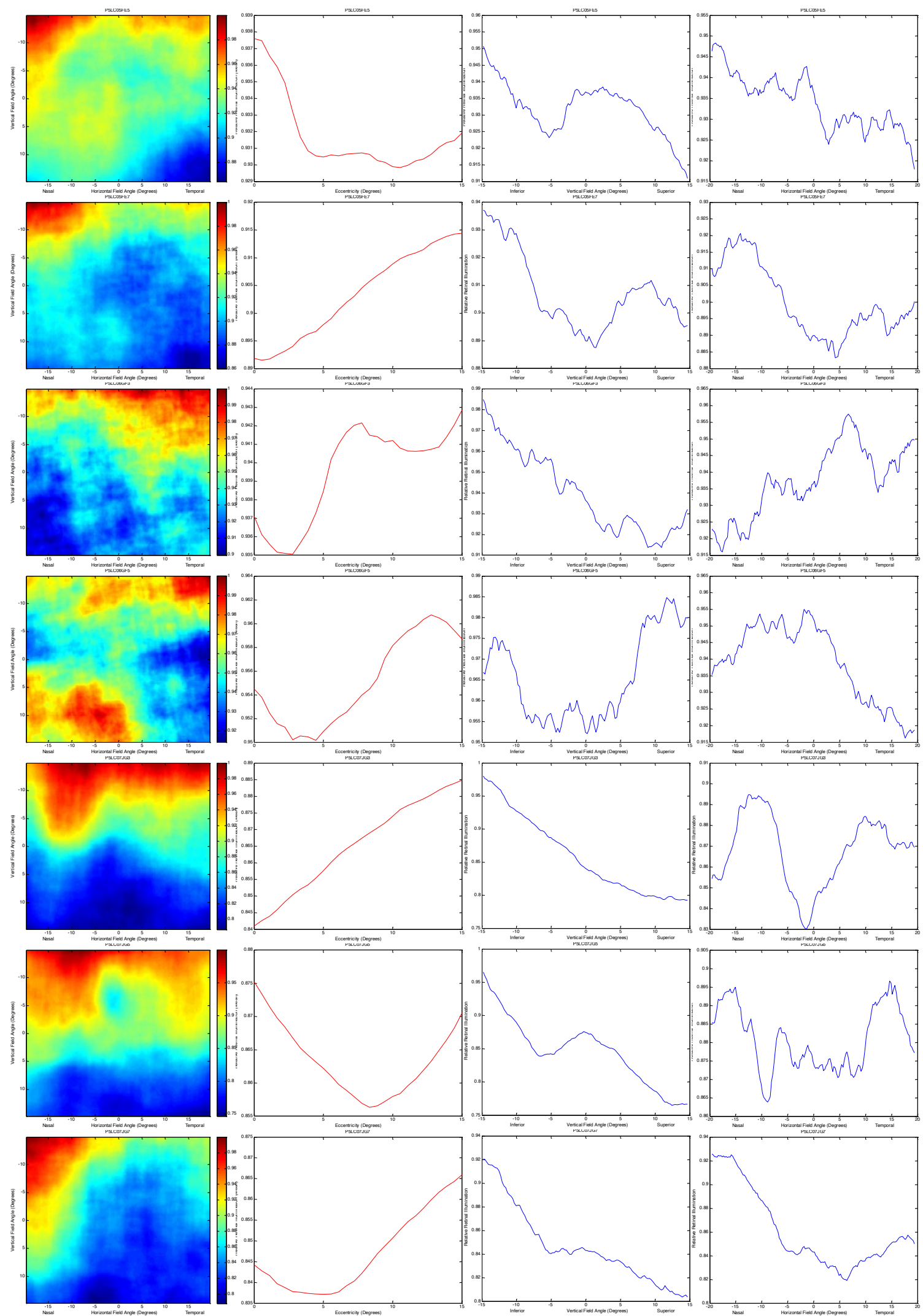

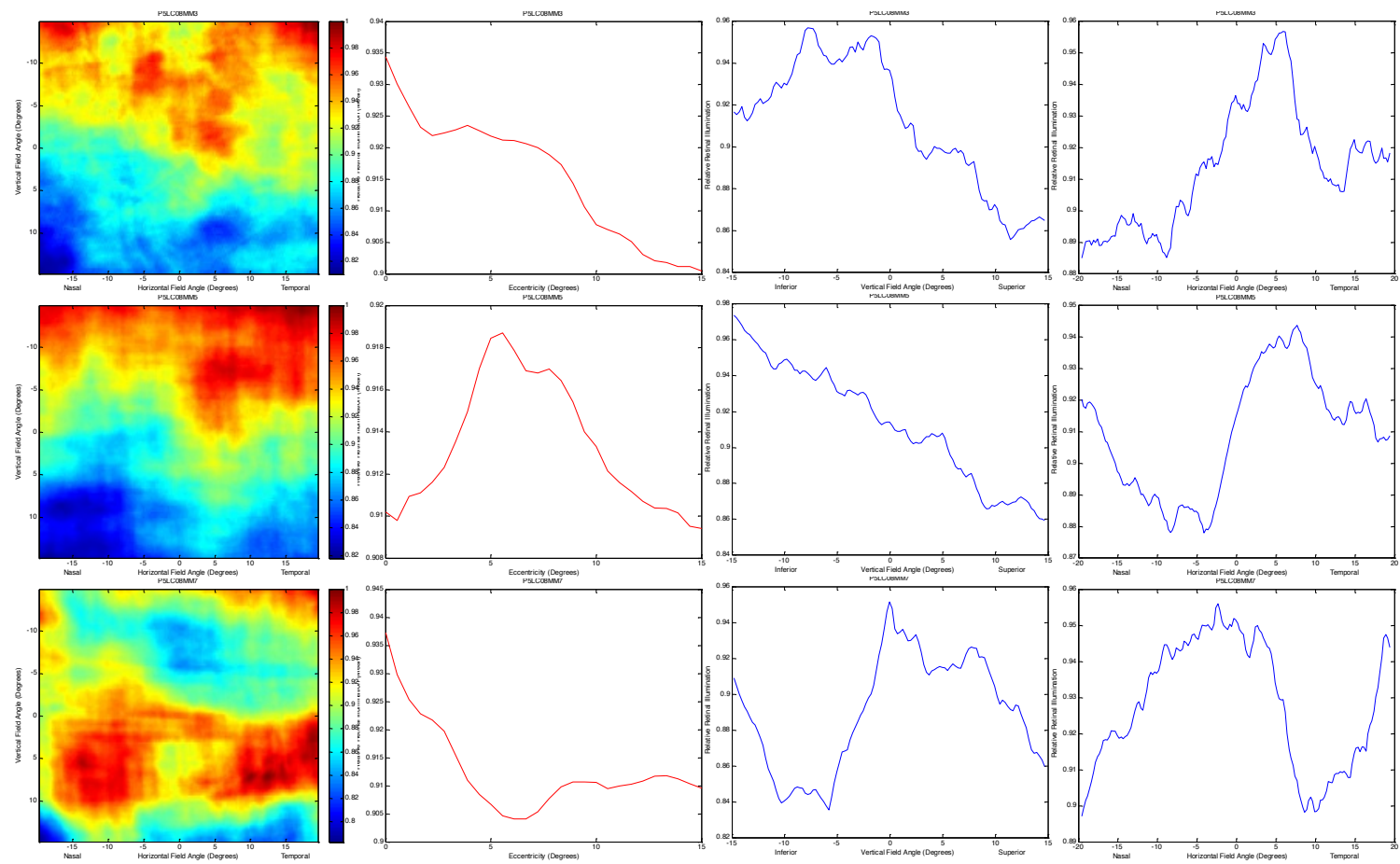


\section{APPENDIX 19}

This appendix contains the individual retinal illumination maps, the horizontal and vertical line-scans across the fovea, and the retinal illumination versus radial eccentricity from the fovea for the subjects that walked freely around the FIU campus in Phase 5 (P5O)
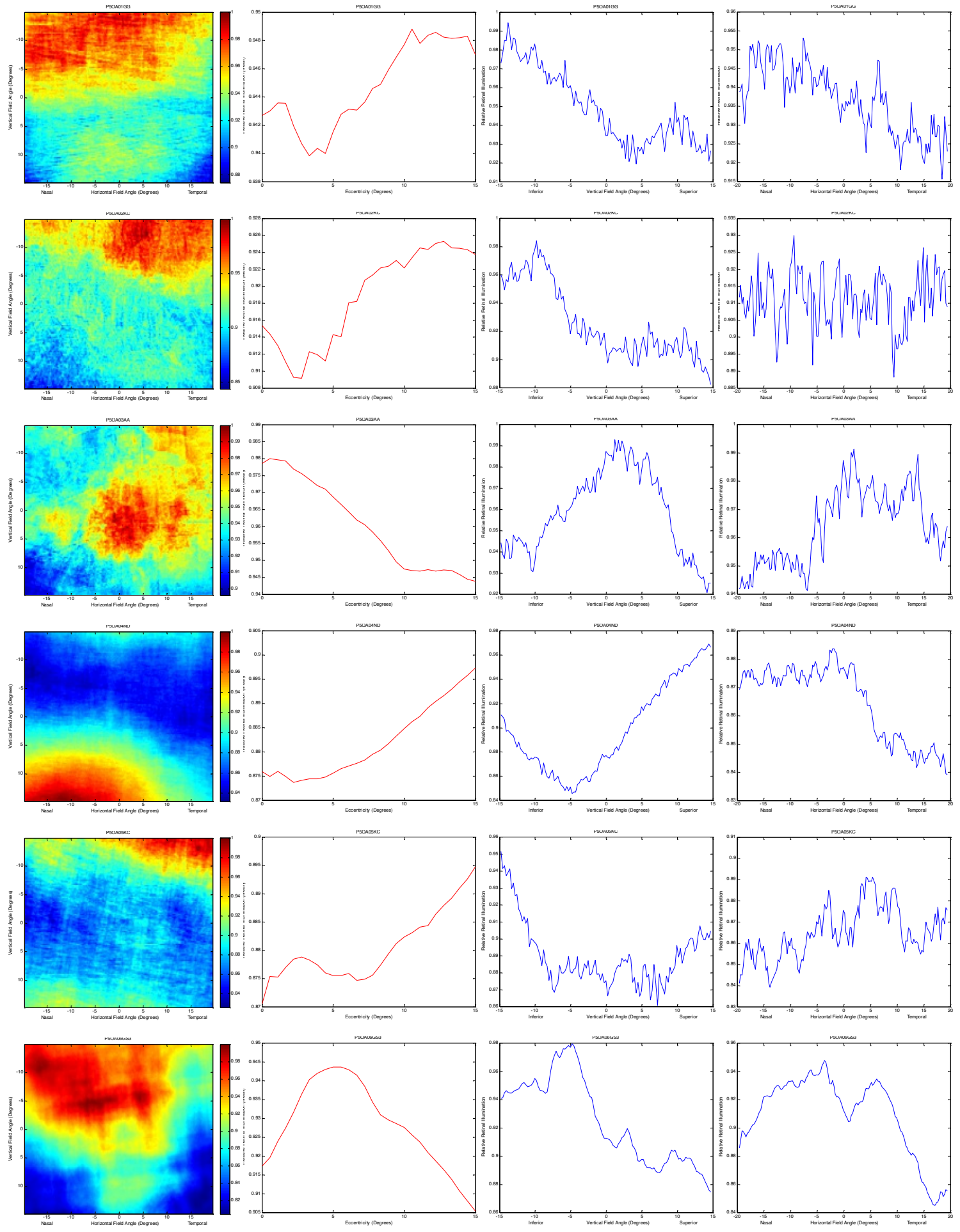

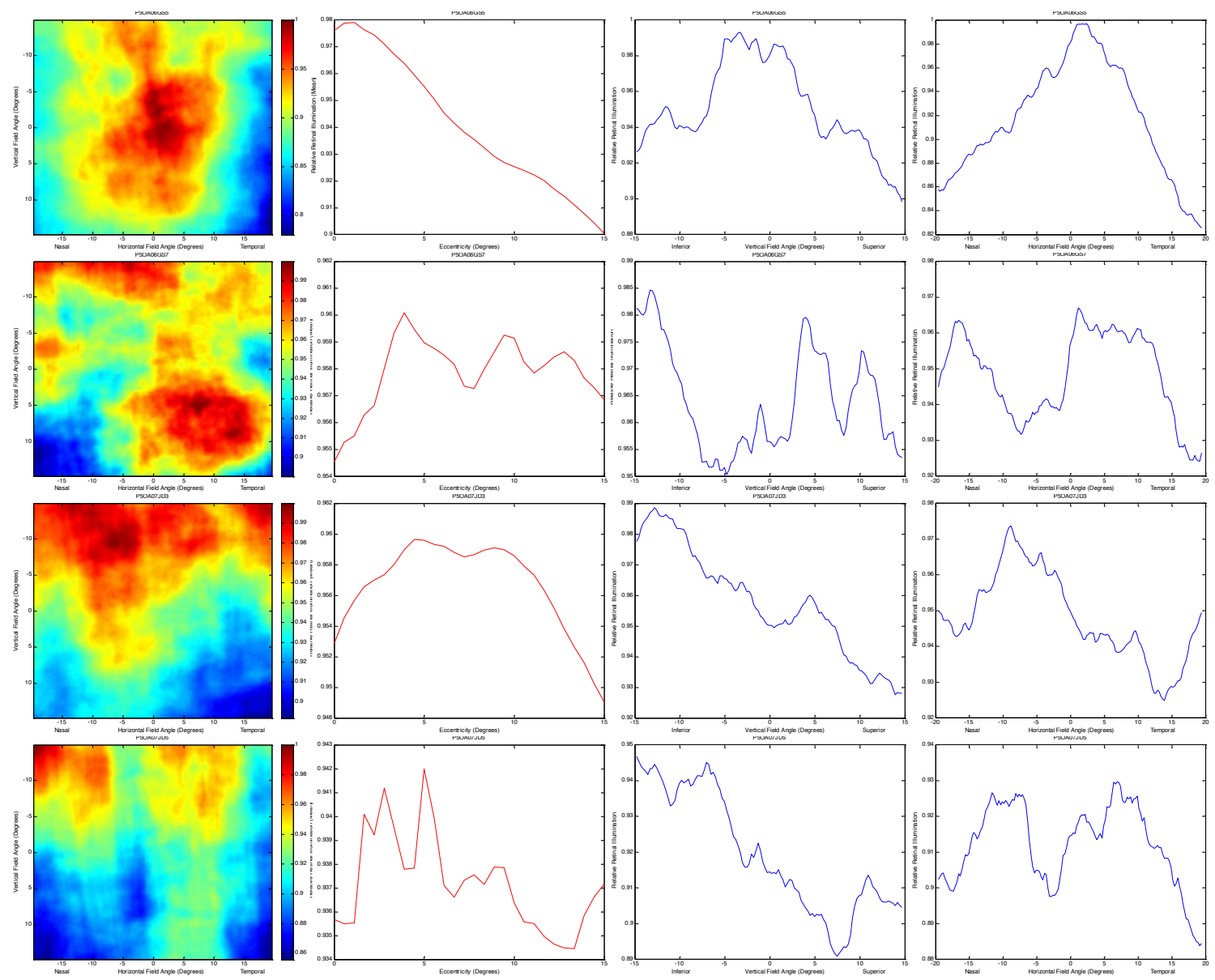


\section{VITA}

\section{JORGE C. GIBERT}

June 21, 1970

1994

1994-1996

1995-1996

1996-2002

1998-2002

2002-2007

2004

2004-2008

2008-2009

2009-2010

2010-Present
Born, Santa Clara, Cuba

B.S., Physics

Universidad de Oriente

Santiago de Cuba, Cuba

Construction Coordinator,

Municipal Directorate of Health

Santa Clara, Cuba

Hemodynamics Trainee

Arnaldo Milian Castro Hospital

Santa Clara, Cuba

Shift Manager

Aircraft Services International Group

Miami, Florida

Teacher Assistant

Florida International University

Miami, Florida

Physics Teacher

Miami Sunset Senior High School

Miami, Florida

M.S., Physics

Florida International University

Miami, Florida

Research Assistant

Florida International University

Miami, Florida

MBRS-RISE Scholarship Recipient

Florida International University

Miami, Florida

Visiting Faculty

Florida International University

Miami, Florida

Teacher Assistant

Florida International University

Miami, Florida

Physics Faculty

Miami-Dade College

Miami, Florida 


\section{PUBLICATIONS}

\section{Refereed Articles}

Bone R.A., Landrum J.T., Gibert J.C. (2004). Macular pigment and the edge hypothesis of flicker photometry. Vision Res. 44, 3045-3051.

Bone R.A., Brener B., Gibert, J.C. (2007). Macular pigment, photopigments, and melanin: distributions determined by four-wavelength reflectometry. Vision Res. 47, 3259-3268.

Bone, R. A., Gibert, J. C., \& Mukherjee, A. (2012). Light distributions on the retina: relevance to macular pigment photoprotection. Acta Biochimica Polonica, 59(1), 91-96.

Bone, R. A., Gibert, J. C., \& Mukherjee, A. (2013) Light distribution on the retina Implications for age-related macular degeneration. In Landrum, J. T. and Nolan J, Carotenoids and Retinal Disease, Taylor \& Francis Group. London

\section{E-Abstracts}

R.A. Bone, J.C. Gibert, J.T. Landrum (2004). Macular pigment and the edge hypothesis of heterochromatic flicker photometry. Invest. Ophthalmol. Vis. Sci. 45: E-Abstract 1294.

J.C. Gibert, R.A. Bone, J.T. Landrum (2004) Macular pigment (as well as cone and rod photopigment) distribution from a single retinal image: a pilot study. Invest. Ophthalmol. Vis. Sci. 45: E-Abstract 1286 .

R.A. Bone, B. Brener, J.C. Gibert, J.T. Landrum, M. Saint-Luis, M.J. Adams (2005) Macular pigment optical density measurements by four-wavelength reflectometry. Invest. Ophthalmol. Vis. Sci. 46: EAbstract 1785 .

R.A. Bone, J.T. Landrum, M.J. Adams, J.C. Gibert (2007) Validity of Macular Pigment Optical Density Measurements by Heterochromatic Flicker Photometry. Invest. Ophthalmol. Vis. Sci. 48: EAbstract 2131

J.C. Gibert, R.A. Bone, J.T. Landrum, M.J. Adams (2007) Distribution of Light on the Retina: A Pilot Study. Invest. Ophthalmol. Vis. Sci. 48: E-Abstract 3697.

J. C. Gibert, R. A. Bone and J. T. Landrum (2008) Cumulative Light Distribution on the Human Retina Determined With an Eye-Tracker. Invest Ophthalmol Vis Sci. 49: E-Abstract 4411

R. A. Bone, J. C. Gibert and J. T. Landrum (2010) Cumulative Light Distribution on the Human Retina under Natural Viewing Conditions. Invest Ophthalmol Vis Sci. 51: E-Abstract 2261.

Jorge C. Gibert, Richard A. Bone, Anirbaan Mukherjee and John T. Landrum (2011) Cumulative Light Distribution on the Human Retina: Implications for AMD. Invest Ophthalmol Vis Sci. 52: EAbstract 382.

Anirbaan Mukherjee, Richard A. Bone and Jorge C. Gibert (2012) Age-Related Decline in Macular Pigment with LED-based Flicker: a Possible Artifact of the Aging Lens. Invest Ophthalmol Vis Sci. 53: E-Abstract 3363 